\title{
The geometric triangle for 3-dimensional Seiberg-Witten monopoles
}

\author{
A.L. Carey, M. Marcolli, B.L. Wang
}

\section{Contents}

1 Introduction 1

2 Seiberg-Witten equations on 3-manifold 4

3 Monopoles on a 3-manifold with a cylindrical end $\quad 10$

3.1 Monopoles on $T^{2} \times[0, \infty) \ldots \ldots \ldots \ldots \ldots$

3.2 Local structure of moduli space of irreducible monopoles . . . . . . . . . 22

3.3 Proof of Theorem 1.2 . . . . . . . . . . . . . . . . . . 29

4 Gluing of 3-dimensional monopoles $\quad \mathbf{3 0}$

4.1 Convergence of monopoles on a 3-manifold with a long neck . . . . . . . 32

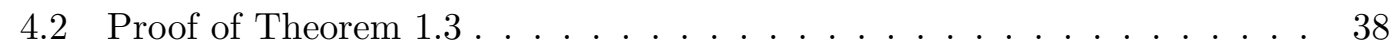

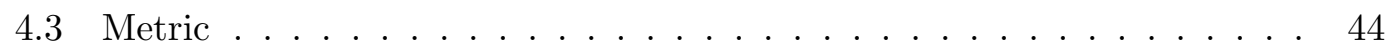

4.4 Lines in $\chi\left(T^{2}\right) \ldots \ldots \ldots \ldots \ldots \ldots \ldots$

$5 \quad$ The geometric triangle and proof of Theorem $1.1 \quad 47$

$\begin{array}{lll}6 & \text { Relative grading } & 49\end{array}$

\section{Introduction}

This paper is the first part of a program aimed at a better understanding of how the recently defined Seiberg-Witten-Floer homology for any closed 3-manifold $Y$ with a $\operatorname{Spin}^{c}$ structure $\mathfrak{s}$ [7], [12], [18], [20], [35] behaves under surgery. The non-equivariant Seiberg-Witten-Floer homology is constructed from the chain complex generated by the irreducible critical points of the perturbed Chern-Simons-Dirac functional on the space of $L_{1}^{2}$-configurations modulo the action of $L_{2}^{2}$-gauge transformations, the differential is defined by counting the gradient flow lines connecting the critical points of relative 
index one. These critical points are the equivalence classes of solutions to the SeibergWitten equations on $(Y, \mathfrak{s})$ modulo gauge transformations. The gradient flowlines are the equivalence classes of solutions to the Seiberg-Witten equations on $Y \times \mathbb{R}$ with the pull-back Spin ${ }^{c}$ structure, modulo gauge transformations. For a general introduction to Seiberg-Witten Floer theory see [20].

Throughout the paper we are considering an oriented, closed homology 3 -sphere $Y$ and a knot $K$ smoothly embedded in $Y$. We consider two other manifolds obtained by Dehn surgery on $K$ : a homology 3 -sphere $Y_{1}$, obtained by +1 -surgery on $K$, and a 3-manifold $Y_{0}$ which has the homology of $S^{1} \times S^{2}$, obtained by 0 -surgery on $K$. Our main goal is to establish the existence of an exact triangle relating the Seiberg-WittenFloer homology groups of these manifolds. A similar setup for instanton homology in Donaldson theory was considered in [2], where Floer's ideas on the corresponding construction of the exact triangle for instanton homology are presented.

Because of various technical difficulties intrinsic in this program, we need to subdivide the problem into several steps. In this first paper we deal with the "geometric triangle", namely we introduce a suitable "surgery perturbation" $\mu$ for the SeibergWitten equations on $Y$ that simulates the effect of surgery. We use the notation $\mathcal{M}_{Y, \mu}$ for the moduli space of gauge classes of solutions of the perturbed Seiberg-Witten equations on $Y, \mathcal{M}_{Y_{1}}$ and $\mathcal{M}_{Y_{0}}(\mathfrak{s})$ for the moduli spaces of the perturbed Seiberg-Witten monopoles on $Y_{1}$ and $\left(Y_{1}, \mathfrak{s}\right)$, where $\mathfrak{s}$ is a $\operatorname{Spin}^{c}$ structure on $Y_{0}$.

Our main result in this paper is to prove the following decomposition theorem for $\mathcal{M}_{Y, \mu}$.

Theorem 1.1. With a careful choice of perturbations and metrics on $Y, Y_{1}$ and $Y_{0}$, we have the following relation between the critical sets of the Chern-Simons-Dirac functional on the manifolds $Y, Y_{1}$ and $Y_{0}$ :

$$
\mathcal{M}_{Y, \mu} \cong \mathcal{M}_{Y_{1}} \cup \bigcup_{\mathfrak{s}_{k}} \mathcal{M}_{Y_{0}}\left(\mathfrak{s}_{k}\right),
$$

where $\mathfrak{s}_{k}$ runs over the $\operatorname{Spin}^{c}$-structures on $Y_{0}$.

In section 2, we will briefly review the perturbation theory we use to define our moduli spaces. In this paper, we only introduce perturbations sufficient to achieve transversality of moduli spaces of critical points. Eventually, when dealing with the full Seiberg-Witten-Floer homology, we shall need a more sophisticated class of perturbations that achieve transversality simultaneously for moduli spaces of critical points and of flow lines. These will be non-local perturbations of the Chern-Simons-Dirac functional, somewhat similar to those proposed in [12]. We shall deal with this more refined perturbation theory elsewhere.

In section 3, we will study the Seiberg-Witten monopoles on the knot complement $V=Y-K$, equipped with a cylindrical end metric modelled on $T^{2} \times[0, \infty)$. We use the 
notation $\chi\left(T^{2}, V\right)$ for the moduli space of flat connections on $T^{2}$ modulo the subgroup of gauge transformations on $T^{2}$ which can be extended to $V$. Notice that $\chi\left(T^{2}, V\right)$ is a $\mathbb{Z}$-covering of the moduli space of flat connections on $T^{2}$ modulo the gauge group $\operatorname{Map}\left(T^{2}, U(1)\right)$ which we denote by $\chi\left(T^{2}\right)$. In $\chi\left(T^{2}\right)$, there is a unique point $\Theta$ such that the Dirac operator on $T^{2}$ coupled with $\Theta$ has non-trivial kernel. The main result in section 2 is the following structure theorem for the monopole moduli space $\mathcal{M}_{V}$.

Theorem 1.2. For generic metrics and perturbations, the moduli space of SeibergWitten monopoles on $V$, denoted by $\mathcal{M}_{V}$, consists of the union of a circle of reducibles $\chi(V)$ and an irreducible piece $\mathcal{M}_{V}^{*}$ which is a smooth oriented 1-dimensional manifold, compact except for finitely many ends limiting to $\chi(V)$. Moreover, there is a continuous boundary value map

$$
\mathcal{M}_{V} \stackrel{\partial_{\infty}}{\rightarrow} \chi\left(T^{2}, V\right) \stackrel{\pi}{\rightarrow} \chi\left(T^{2}\right)
$$

defined by taking the asymptotic limit of the Seiberg-Witten monopoles on $V$ over the end. Under $\partial_{\infty}, \chi(V)$ is mapped to a circle in $\chi\left(T^{2}, V\right)$, and the compactification $\overline{\mathcal{M}}_{V}^{*}$ of $\mathcal{M}_{V}^{*}$ is mapped to a collection of compact immersed curves in $\chi\left(T^{2}, V\right)$ whose boundary points consist of a finite set of points in $\pi^{-1}(\Theta) \cup \partial_{\infty}(\chi(V))$. For generic perturbations the interior of the curve $\partial_{\infty}\left(\mathcal{M}_{V}^{*}\right)$ is transverse to any given finite set of curves in $\chi\left(T^{2}, V\right)$.

For simplicity of notation, in the following we shall not distinguish between $\chi(V)$ and its embedded image $\partial_{\infty}(\chi(V)) \subset \chi\left(T^{2}, V\right)$.

In section 4 , we will establish a gluing theorem for the moduli spaces of critical points of the Chern-Simons-Dirac functional when cutting and gluing the 3-manifold along a torus. In our case, these are the moduli spaces of monopoles on a closed manifold which is either $Y, Y_{1}$, or $Y_{0}$. Let $\nu(K)$ be a tubular neighbourhood of $K$ in a closed manifold $Z$, so $Z=V \cup \nu(K)$. We may cut $Z$ along $T^{2}$ and glue in a long cylinder $[-r, r] \times T^{2}$, resulting in a new manifold denoted by $Z(r)$. Use the notation $\chi\left(T^{2}, Z\right)$ for the character variety (or moduli space) of flat connections on a trivial line bundle over $T^{2}$ modulo the gauge transformations on $T^{2}$ which can be extended to $Z$. We denote by $\chi(\nu(K), Z)$, the moduli space of flat connections on $\nu(K)$ modulo the gauge transformations on $\nu(K)$ which can be extended to $Z$. there is a natural map $\chi(\nu(K), Z) \rightarrow \chi\left(T^{2}, Z\right)$. We denote by $\mathcal{M}_{V, Z}^{*}$ the moduli space of the Seiberg-Witten monopoles on $V$ modulo the gauge transformations on $V$ which can be extended to $Z$. We have a refinement of the boundary value map of (2):

$$
\mathcal{M}_{V, Z}^{*} \longrightarrow \chi\left(T^{2}, Z\right)
$$

Then we have the following gluing theorem. 
Theorem 1.3. For a sufficiently large $r$, under suitable perturbations and choice of metrics, there exist the following diffeomorphisms given by the gluing maps on the fibered products

$$
\begin{gathered}
\#_{Y}: \mathcal{M}_{V, Y}^{*} \times_{\chi\left(T^{2}, Y\right)} \chi(\nu(K), Y) \longrightarrow \mathcal{M}_{Y(r)}^{*}, \\
\#_{Y_{1}}: \mathcal{M}_{V, Y_{1}}^{*} \times_{\chi\left(T^{2}, Y_{1}\right)} \chi\left(\nu(K), Y_{1}\right) \longrightarrow \mathcal{M}_{Y_{1}(r)}^{*}, \\
\#_{Y_{0}}: \mathcal{M}_{V, Y_{0}}^{*} \times_{\chi\left(T^{2}, Y_{0}\right)} \chi\left(\nu(K), Y_{0}\right) \longrightarrow \bigcup_{\mathfrak{s}} \mathcal{M}_{Y_{0}(r)}^{*}(\mathfrak{s}) .
\end{gathered}
$$

Here, $\mathcal{M}_{Y(r)}^{*}, \mathcal{M}_{Y_{1}(r)}^{*}$ and $\mathcal{M}_{Y_{0}(r)}^{*}$ are the moduli spaces of irreducible monopoles on $Y(r), Y_{1}(r)$ and $Y_{0}(r)$ respectively, and $\mathfrak{s}$ runs over all the possible $\operatorname{Spin}^{c}$ structures on $Y_{0}$. The fiber product is taken with respect to the refined boundary value maps (3) from $\mathcal{M}_{V, Y}^{*}, \mathcal{M}_{V, Y_{1}}^{*}$ and $\mathcal{M}_{V, Y_{0}}^{*}$ to $\chi\left(T^{2}, Y\right), \chi\left(T^{2}, Y_{1}\right)$ and $\chi\left(T^{2}, Y_{0}\right)$ respectively.

The proof of Theorem 1.3 is based on balancing the slow decay of certain eigenfunctions of the linearization at the approximate solutions, against the exponential decay of the finite energy solutions on $V$ with non-degenerate asymptotic value, thus obtaining an unobstructed gluing.

Using the gluing Theorem 1.3, together with the construction of the perturbation $\mu$ that "simulates the effect of surgery", we will be able to derive a corresponding deformation of the moduli spaces, and the expected relation between the generators of the Floer groups as in Theorem 1.1.

In the last section, we apply the result of Capell-Lee-Miller on the decomposition of spectral flow (Theorem $\mathrm{C}$ of [4]) to study the relative gradings of monopoles under the identification of Theorem 1.1. We show that the identification of Theorem 1.1 is compatible with the relative gradings on the Seiberg-Witten-Floer chain complexes (Cf. Proposition 6.2 and Proposition 6.4).

Acknowledgments We are very grateful to Ronnie Lee, Tom Mrowka, Vicente Munoz, and Peter Ozsvath for useful discussions and suggestions. We like to thank Cliff Taubes for providing the proof of Lemma 2.3 and Liviu Nicolaescu for the proof of Lemma 4.11. The three authors also thank the Max-Planck-Institut für Mathematik, Bonn for the kind hospitality and for support. AC and BW are partially supported by Australian Research Council. MM is partially supported by NSF grant DMS-9802480 and by Humboldt Foundation (Sofja Kovalevskaya Award).

\section{Seiberg-Witten equations on 3-manifold}

The 3-dimensional Seiberg-Witten monopoles on a compact manifold have been extensively studied in [6] [8] [11] [13] [14] [20] [21] [24]. In this section we will briefly recall some of the main features of 3-dimensional monopoles. A 3-dimensional monopole, as noted first in [13], can be viewed as a critical point of the Chern-Simon-Dirac functional 
on an infinite dimensional space (the orbit space of $\operatorname{Spin}^{c}$ connections and sections of the spinor bundle under the action of the gauge group). We recall the basic setting of 3-dimensional Seiberg-Witten theory, then we will end this section with the observation that, under a generic perturbation with compact support in a fixed open set, the critical points are all non-degenerate.

Let $(Y, g)$ be a closed, oriented Riemannian 3-manifold. A Spin ${ }^{c}$ structure $\mathfrak{s}$ on $(Y, g)$ is a pair $(W, \rho)$ consisting of a rank 2 Hermitian bundle $W$ together with a Clifford multiplication $\rho: T^{*} Y \rightarrow \operatorname{End}(W)$. If $\left\{e_{1}, e_{2}, e_{3}\right\}$ are an oriented orthonormal frame for $T Y$, we choose the Clifford multiplication such that $\rho\left(e_{1}\right) \rho\left(e_{2}\right) \rho\left(e_{3}\right)=1$.

With the Levi-Civita connection on the frame bundle of $Y$, a $U(1)$-connection $A$ on the determinant bundle $\operatorname{det}(W)$ determines a $\operatorname{Spin}^{c}$ connection $\nabla_{A}$ on $W$ such that $\rho$ is parallel. Applying Clifford multiplication, we can define a Dirac operator, denoted by $\not_{A}$. Then the Seiberg-Witten equations are the equations for a pair $(A, \psi)$ consisting of a $U(1)$-connection on $\operatorname{det}(W)$ and a section $\psi$ of $W$ ( $\psi$ is called a spinor):

$$
\left\{\begin{array}{l}
* F_{A}=\sigma(\psi, \psi)+\mu \\
\not_{A}(\psi)=0
\end{array}\right.
$$

Here $\mu$ is a co-closed imaginary-valued 1 -form on $Y$, and $\sigma(\cdot, \cdot)$ is a symmetric $\mathbb{R}$-bilinear form $W \otimes W \rightarrow T^{*} Y \otimes i \mathbb{R}$ given by

$$
\begin{aligned}
\sigma(\psi, \psi) & =-\rho^{-1}\left(\left(\psi \otimes \psi^{*}\right)_{0}\right)=-\rho^{-1}\left(\psi \otimes \psi^{*}-\frac{|\psi|^{2}}{2} I d\right) \\
& =\frac{i}{2} \operatorname{Im}\left\langle\rho\left(e_{i}\right) \psi, \psi\right\rangle e^{i}
\end{aligned}
$$

Note that this $\mathbb{R}$-bilinear form $\sigma(\cdot, \cdot)$ satisfies the following property [7]:

(1) Under Clifford multiplication, we have $\sigma(\psi, \psi) \cdot \psi=-\frac{1}{2}|\psi|^{2} \psi$, and $\langle\alpha . \psi, \psi\rangle=2\langle\alpha, \sigma(\psi, \psi)\rangle_{T^{*} Y}$, for $\alpha \in \Omega^{1}(Y, i \mathbb{R})$.

(2) $\sigma(\psi, \phi)=0$ if and only if on $Y-\psi^{-1}(0) \phi=i r \psi$ for a real-valued function $r$ on $Y-\psi^{-1}(0)$.

(3) For any imaginary valued 1-form $\alpha, \sigma(\alpha . \psi, \phi)+\sigma(\psi, \alpha . \phi)=-(\operatorname{Re}\langle\psi, \phi\rangle) \alpha$.

(4) If $\psi$ is a nowhere vanishing section of $W$, then $W \cong \mathbb{C} \psi \oplus \psi^{\perp}$, and $\sigma(\psi, \cdot)$ defines a bundle isomorphism between $\underline{\mathbb{R}} \psi \oplus \psi^{\perp}$ and $T^{*} Y \otimes i \mathbb{R}$.

Denote by $\mathcal{A}_{Y}$ the configuration space of $(Y, \mathfrak{s})$ consisting of pairs $(A, \psi)$ with the completion under $L_{1}^{2}$-norm. The gauge group of automorphisms of the $\operatorname{Spin}^{c}$-bundle $W$ is $\mathcal{G}_{Y}=\operatorname{Map}(Y, U(1))$ with $L_{2}^{2}$-completion. $\mathcal{G}_{Y}$ acts on $\mathcal{A}_{Y}$ by

$$
u(A, \psi)=\left(A-2 u^{-1} d u, u \psi\right),
$$

and the Seiberg-Witten equations are invariant under this action. Denote by $\mathcal{B}_{Y}$ the quotient space of $\mathcal{A}_{Y}$ by the gauge group action. $\mathcal{B}_{Y}$ is an infinite dimensional Hilbert 
manifold except at points where the spinor part is zero, which are called reducible points. Otherwise, points $(A, \psi)$ with $\psi \neq 0$ are called irreducible. As noted in [13], the Seiberg-Witten equations on $(Y, \mathfrak{s}, g)$ are the equations for the critical points of the following Chern-Simons-Dirac functional on $\mathcal{A}_{Y}$ :

$$
\mathcal{C}_{\mu}(A, \psi)=-\frac{1}{2} \int_{Y}\left(A-A_{0}\right) \wedge\left(F_{A}+F_{A_{0}}-2 * \mu\right)+\int_{Y}\left\langle\psi, \not_{A} \psi\right\rangle d v o l_{Y},
$$

where $A_{0}$ is a fixed connection on $\operatorname{det}(W)$. Note that $\mathcal{C}_{\mu}$ descends to a circle-valued function on $\mathcal{B}_{Y}$. The set of critical points of $\mathcal{C}_{\mu}$ on $\mathcal{B}_{Y}$ is denoted by $\mathcal{M}_{Y, \mu}(\mathfrak{s})$, its irreducible critical point set is denoted by $\mathcal{M}_{Y, \mu}(\mathfrak{s})^{*}$.

For any critical point $(A, \psi)$ on $\mathcal{A}_{Y}$, the infinitesimal action of $\mathcal{G}_{Y}$ and the derivative of $\operatorname{grad}\left(\mathcal{C}_{\mu}\right)$ at $(A, \psi)$ define a complex

$$
\Omega_{L_{2}^{2}}^{0}(Y, i \mathbb{R}) \stackrel{G_{(A, \psi)}}{\rightarrow} \Omega_{L_{1}^{2}}^{1}(Y, i \mathbb{R}) \oplus L_{1}^{2}(W) \stackrel{H_{(A, \psi)}}{\longrightarrow} \Omega_{L^{2}}^{1}(Y, i \mathbb{R}) \oplus L^{2}(W),
$$

where the maps $G_{(A, \psi)}$ and $H_{(A, \psi)}$ are given by

$$
\begin{aligned}
& G_{(A, \psi)}(f)=(-2 d f, f \psi), \\
& H_{(A, \psi)}(\alpha, \phi)=\left(* d \alpha-2 \sigma(\psi, \phi), \partial_{A} \phi+\frac{1}{2} \alpha . \psi\right) .
\end{aligned}
$$

We say that $[A, \psi]$ is a non-degenerate critical point of $\mathcal{C}_{\mu}$ on $\mathcal{B}_{Y}$ if the middle cohomology of (6) is zero:

$$
\operatorname{KerH}_{(A, \psi)} / \operatorname{Im}_{(A, \psi)}=0 .
$$

At the smooth points of $\mathcal{B}_{Y}$, this definition is the same as saying that the derivative of $\operatorname{grad}\left(\mathcal{C}_{\mu}\right)$ at a critical point is non-degenerate. The gradient of $\mathcal{C}_{\mu}$ can be viewed as an $L^{2}$-tangent vector field on $\mathcal{B}_{Y}$, a section of the $L^{2}$-tangent bundle over $\mathcal{B}_{Y}$, while the tangent space of $\mathcal{B}_{Y}$ at $[A, \psi]$ is the $L_{1}^{2}$-completion of

$$
\operatorname{Ker} G_{(A, \psi)}^{*}=\left\{(\alpha, \phi) \mid d^{*} \alpha+i \operatorname{Im}\langle\psi, \phi\rangle=0 .\right\}
$$

The covariant derivative of $\operatorname{grad}\left(\mathcal{C}_{\mu}\right)$, denoted by $H_{[A, \psi]}$, defines a operator on $\operatorname{Ker} G_{(A, \psi)}^{*}$, sending $(\alpha, \phi) \in \operatorname{Ker} G_{(A, \psi)}^{*}$ to

$$
\left(* d \alpha-2 \sigma(\psi, \phi)-2 d f, \not_{A} \phi+\frac{1}{2} \alpha . \psi+f \psi\right),
$$

where $f$ is the unique solution to the equation

$$
\left(d^{*} d+\frac{1}{2}|\psi|^{2}\right) f=i \operatorname{Im}\left\langle\partial_{A} \psi, \phi\right\rangle .
$$

Note that $H_{[A, \psi]}$ is a closed, unbounded, essentially self-adjoint, Fredholm operator on the $L^{2}$-completion of $\operatorname{Ker} G_{(A, \psi)}^{*}$, its eigenvectors form an $L^{2}$-complete orthonormal 
basis, its $L^{2}$-spectrum forms a discrete subset of the real line with no accumulation points. Hence, as in [20], the spectral flow of $H_{[A, \psi]}$, along a path connecting two critical points defines a relative index on $\mathcal{M}_{Y, \mu}^{*}(\mathfrak{s}) \times \mathcal{M}_{Y, \mu}^{*}(\mathfrak{s})$. This relative index depends only on the homotopy class of the connecting path for non-torsion $\operatorname{Spin}^{c}$ structure (Cf. Remark 4.5 in [20] and Definition 3.6 in [7]).

The following properties about the critical points of $\mathcal{C}_{\mu}$ are now standard (See [20] [8] [11] [14] [16]).

Proposition 2.1. There exists a Baire set of co-closed 1-form $\mu \in \Omega_{L_{2}^{2}}^{1}(Y, i \mathbb{R})$ such that all the critical points in $\mathcal{M}_{Y, \mu}(\mathfrak{s})$ are non-degenerate. Moreover, if $b_{1}(Y)>0$, $\mathcal{M}_{Y, \mu}(\mathfrak{s})$ consists of only finitely many irreducible points in $\mathcal{B}_{Y}$; if $Y$ is a rational homology 3-sphere, assume that a generic $\mu$ satisfies $\operatorname{Ker} \not_{\theta}=0$ (where $\theta$ is the unique reducible point in $\mathcal{M}_{Y, \mu}(\mathfrak{s})$, that is, $\left.* F_{\theta}=\mu\right)$, then $\mathcal{M}_{Y, \mu}(\mathfrak{s})^{*}=\mathcal{M}_{Y, \mu}(\mathfrak{s})-\{\theta\}$ consists of only finitely many irreducible points.

In this paper and sequel work, it is convenient to use a perturbation with support contained in a fixed open set, so that Proposition 2.1 still holds for perturbations with compact support contained in a fixed open set. The first such statement was made in Proposition 7.1 [32] by Taubes, who kindly communicated the proof to us.

Proposition 2.2. Fix a non-empty open set $U$ in $Y$ and a $\operatorname{Spin}^{c}$ structure $\mathfrak{s}$ on $Y$, if $b_{1}(Y)>0$ and $c_{1}(\operatorname{det}(\mathfrak{s}))=0$, we require that $U$ is chosen so that the map $H^{2}(Y, \mathbb{R}) \rightarrow H^{2}(U, \mathbb{R})$ is non-zero. Then there exists a Baire set of co-closed imaginary valued 1-forms $\mu$ with compact support in $U$ such that all the critical points of $\mathcal{C}_{\mu}$ on $\mathcal{B}_{Y}$ are non-degenerate.

Proof. We first study the family version of the critical points of $\mathcal{C}_{\mu}$ on $\mathcal{B}_{Y}^{*}$, where $\mu$ is from a set of imaginary valued co-closed 1-forms on $Y$ with compact support in $U$. Denote this set of perturbations as $Z(U, i \mathbb{R})$. Let $[\mu, A, \psi]$ be a critical point of $\mathcal{C}_{\mu}$. We need to show that the derivative of the gradient of $\left\{\mathcal{C}_{\mu}\right\}_{\mu \in Z(U, i \mathbb{R})}$ is surjective. Namely, consider

$$
\operatorname{Ker} G_{[A, \psi]}^{*} \times Z(U, i \mathbb{R}) \rightarrow \operatorname{Ker} G_{[A, \psi]}^{*},
$$

which sends $\left(\alpha, \phi, \mu_{1}\right)$ to

$$
\left(* d \alpha-2 \sigma(\psi, \phi)+\mu_{1}, \not \partial_{A} \phi+\frac{1}{2} \alpha \cdot \psi\right)
$$

Suppose that $(\alpha, \phi)$ is orthogonal to the image of the above map, then $(\alpha, \phi)$ satisfies 
the following equations:

$$
\begin{aligned}
& \text { (1) } d^{*} \alpha+i \operatorname{Im}\langle\psi, \phi\rangle=0, \\
& \text { (2) } * d \alpha-2 \sigma(\psi, \phi)=0, \\
& \text { (3) } \not_{A} \phi+\frac{1}{2} \alpha \cdot \psi=0,
\end{aligned}
$$

(4) $\alpha$ is exact when restricted to $U$.

The elliptic regularity implies that $(\alpha, \phi)$ is smooth. From (4) and (2) of equations (7), we know that $\sigma(\psi, \phi)=0$ on $U$. The following Lemma due to Taubes [33] will ensure that $\sigma(\psi, \phi)=0$ on $Y$. Hence, there is a real-valued smooth function $\mathrm{f}$ on $Y$, such that $\phi=i f \psi$. Using (3) of (7), we obtain

$$
\not_{A}(i f \psi)+\frac{1}{2} \alpha \cdot \psi=0
$$

on $Y$, which leads to $\alpha=-2 i d f$ on $Y$. By the equation (1) in (7), we get

$$
2 d^{*} d f+f|\psi|^{2}=0
$$

on $Y$. Note that $\psi^{-1}(0)$ does not disconnect any domain in $Y$ (the unique continuation principle for Dirac operator (see page 57-58 [10])). Therefore, $f=0$ which implies that $(\alpha, \phi)=0$.

From the Sard-Smale theorem, there is a Baire set of $\mu \in Z(U, i \mathbb{R})$ such that all critical points of $\mathcal{C}_{\mu}$ in $\mathcal{B}_{Y}^{*}$ are non-degenerate for a generic $\mu$.

Now we need to prove that the reducible critical point of $\mathcal{C}_{\mu}$ is also non-degenerate. By the assumption, $\mathcal{C}_{\mu}$ admits reducible critical point if and only if $Y$ is a rational homology 3-sphere. From the analysis in [20], we know that, in order to achieve the non-degenerate condition at reducible critical point, $\mu$ is required to be away from the codimension one subset $Z(U, i \mathbb{R})$ where the corresponding Dirac operator has nontrivial kernel. This completes the proof of the Proposition. Now we give the proof of Taubes' Lemma.

Lemma 2.3. (Taubes) Let $(A, \psi)$ and $(\alpha, \phi)$ as above, where $(A, \psi)$ is a solution to the Seiberg-Witten equation (4) and $(\alpha, \phi)$ satisfies (1)-(3) of the equations (7). Then $q=\sigma(\psi, \phi)$ obeys an equation of the form

$$
\Delta q=H \cdot q+K \cdot \nabla q
$$

at all points where $\psi \neq 0$. Here $\Delta$ is the Laplacian on differential 1-forms and $H$ and $K$ are linear maps that depend implicitly on $\psi$. The set of points where $\psi \neq 0$ is path connected open dense set in $Y$. The unique continuation principle applies to $q$ so that $q$ cannot vanish on $U$ without vanishing everywhere on $Y$. 
Proof of Taubes' Lemma: Apply the Laplacian to $q=\sigma(\psi, \phi)$, we have the following expression of $\Delta q$ :

$$
\Delta q=\sigma(\Delta \psi, \phi)+\sigma(\psi, \Delta \phi)+2 \sigma\left(\left\{\nabla_{A} \psi, \nabla_{A} \phi\right\}_{T^{*} Y}\right),
$$

here $\Delta$ acting on spinors is $\nabla_{A}^{*} \nabla_{A}$, and $\left\{\nabla_{A} \psi, \nabla_{A} \phi\right\}_{T^{*} Y}$ is the pairing using the metric on $T^{*} Y$. Now invoke the Weitzenböck formula for the Dirac operator,

$$
\not_{A}^{*} \partial_{A}=-\Delta+\frac{\kappa}{4}-\frac{1}{2} \rho\left(* F_{A}\right),
$$

where $\kappa$ is the scalar curvature on $Y$. Thus, from the Dirac equations for $\psi$ and $\phi$, we obtain

$$
\begin{aligned}
& \Delta \psi=\frac{\kappa}{4} \psi-\frac{1}{2}\left(* F_{A}\right) \cdot \psi, \\
& \Delta \phi=\frac{\kappa}{4} \phi-\frac{1}{2}\left(* F_{A}\right) \cdot \phi+\frac{1}{2}\left(d^{*} \alpha\right) \psi-\frac{1}{2}(* d \alpha) \cdot \psi-\nabla_{A}^{\alpha} \psi,
\end{aligned}
$$

here $\nabla_{A}^{\alpha} \psi=\left\{\alpha, \nabla_{A} \psi\right\}_{T^{*} Y}$. Plug these two equations into (8), and note that $\sigma\left(\psi, \frac{1}{2}\left(d^{*} \alpha\right) \psi\right)=0$ and $\sigma\left(\psi,-\frac{1}{2}(* d \alpha) \cdot \psi\right)=|\psi|^{2} q$. We get

$$
\begin{aligned}
\Delta q= & \left(\frac{\kappa}{2}+|\psi|^{2}\right) q+\sigma\left(-\frac{1}{2}\left(* F_{A}\right) \cdot \psi, \phi\right)+\sigma\left(\psi,-\frac{1}{2}\left(* F_{A}\right) \cdot \phi\right) \\
& +2 \sigma\left(\left\{\nabla_{A} \psi, \nabla_{A} \phi\right\}_{T^{*} Y}\right)+\sigma\left(\psi,-\nabla_{A}^{\alpha} \psi\right) \\
= & \left(\frac{\kappa}{2}+|\psi|^{2}\right) q+* F_{A}(\operatorname{Re}\langle\psi, \phi\rangle) \\
& +2 \sigma\left(\left\{\nabla_{A} \psi, \nabla_{A} \phi\right\}_{T^{*} Y}\right)+\sigma\left(\psi,-\nabla_{A}^{\alpha} \psi\right) .
\end{aligned}
$$

Write $\phi=i r \psi+\lambda$ where $r$ is a real-valued function on $Y$ and $\operatorname{Re}\langle\lambda, i \psi\rangle=0$, then

$$
\begin{gathered}
\operatorname{Re}\langle\psi, \phi\rangle=\operatorname{Re}\langle\psi, \lambda\rangle, \\
\sigma\left(\left\{\nabla_{A} \psi, \nabla_{A} \phi\right\}_{T^{*} Y}\right)=\sigma\left(\left\{\nabla_{A} \psi, \nabla_{A} \lambda\right\}_{T^{*} Y}\right)+\sigma\left(\left\{\nabla_{A} \psi, i d r \otimes \psi\right\}_{T^{*} Y}\right) .
\end{gathered}
$$

Hence the equation (9) can be written as

$$
\begin{aligned}
\Delta q= & \left(\frac{\kappa}{2}+|\psi|^{2}\right) q+* F_{A}(\operatorname{Re}\langle\psi, \lambda\rangle) \\
& +2 \sigma\left(\left\{\nabla_{A} \psi, \nabla_{A} \lambda\right\}_{T^{*} Y}\right)+\sigma\left(\psi,-\nabla_{A}^{\alpha+2 i d r} \psi\right) .
\end{aligned}
$$

To complete the proof, we only need to show that $\lambda, \nabla_{A} \lambda$ and $\alpha+2 i d r$ can be written as combinations of linear maps on $q$ and $\nabla q$. On the set of points where $\psi \neq 0, \Omega=Y-\psi^{-1}(0)$, we write $\psi=|\psi| \tau_{1}$ where $\tau_{1}$ is a unit-length spinor. Choose a local basis $\left\{\tau_{1}, \tau_{2}\right\}$ for the $\operatorname{Spin}^{c}$ bundle, so that Clifford multiplication in the local orthonormal coframe $\left\{e^{1}, e^{2}, e^{3}\right\}$ for $T^{*} Y$ is given by

$$
\rho\left(e_{1}\right)=\left(\begin{array}{rr}
i & 0 \\
0 & -i
\end{array}\right), \rho\left(e_{2}\right)=\left(\begin{array}{rr}
0 & -1 \\
1 & 0
\end{array}\right), \rho\left(e_{3}\right)=\left(\begin{array}{ll}
0 & i \\
i & 0
\end{array}\right) .
$$


where $\left\{e^{1}, e^{2}, e^{3}\right\}$ can be expressed as

$$
e^{1}=-2 i \sigma\left(\tau_{1}, \tau_{1}\right), e^{2}=2 i \sigma\left(\tau_{1}, i \tau_{1}\right), e^{3}=-2 i \sigma\left(\tau_{1}, \tau_{2}\right) .
$$

Write $\lambda=u \tau_{1}+v \tau_{2}$ for a real-valued function $u$ and a complex-valued function $v$, then

$$
q=\sigma(\psi, \phi)=\frac{i}{2}|\psi|\left(u e^{1}-\operatorname{Im}(v) e^{2}+\operatorname{Re}(v) e^{3}\right) .
$$

On $\Omega, \sigma_{\psi}=\sigma(\psi, \cdot)$ defines a bundle isomorphism between

$$
\underline{\mathbb{R}} \cdot \psi \oplus(\underline{\mathbb{C}} \cdot \psi)^{\perp} \rightarrow T^{*} Y \otimes i \mathbb{R} .
$$

Thus, we obtain that $\lambda=\sigma_{\psi}^{-1}(q)$, and

$$
\nabla \lambda=\left(\nabla\left(\sigma_{\psi}^{-1}\right)\right)(q)+\sigma_{\psi}^{-1}(\nabla q) .
$$

Let $b=a+2 i d r$, then from (3) of the equations (7), we have

$$
b . \psi=-2 \not \partial_{A} \lambda,
$$

as $\lambda$ can be written in terms of $q$ and $\nabla q$, so is $b$. This completes the proof of Taubes' Lemma.

\section{Monopoles on a 3-manifold with a cylindrical end}

In this section we use techniques developed in [23] to study the moduli space of SeibergWitten monopoles on the knot complement $V$ endowed with an infinite cylindrical end $T^{2} \times[0, \infty)$. Our main aim is to present the proof of Theorem 1.1. Before we give details, we present an overview of the section introducing notation.

Consider the three-manifolds $V$ and $\nu(K)$, respectively the knot complement and the tubular neighbourhood of the knot $K$ in the homology sphere $Y$. Both are 3manifolds with boundary a torus $T^{2}$. Equip $V$ with a cylindrical end metric and a Spin $^{c}$-structure with trivial determinant along the half cylinder $T^{2} \times[0, \infty)$. On $T^{2}$ we use the standard flat metric induced from $\mathbb{R}^{2}$.

The perturbed Seiberg-Witten equations on $(V, \mathfrak{s})$ are the equations

$$
\left\{\begin{array}{l}
* F_{A}=\sigma(\psi, \psi)+\mu, \\
\not_{A} \psi=0,
\end{array}\right.
$$

for a pair $(A, \psi)$ consisting of a $L_{1, l o c}^{2} U(1)$ connection on $\operatorname{det}(W)$ and a $L_{1, l o c}^{2}$ spinor section $\psi$ of $W$. the perturbation term $\mu$ is a co-closed and imaginary value 1 -form 
with compact support contained in a fixed open set $U \subset V-\left(T^{2} \times[0, \infty)\right)$. We denote the corresponding class of perturbations by $Z(U, i \mathbb{R})$

We define the energy of any Seiberg-Witten monopole $(A, \psi)$ to be

$$
\int_{V}\left|F_{A}\right|^{2} d v o l_{V}<\infty
$$

Let $\mathcal{M}_{V}$ denote the Seiberg-Witten moduli space of solutions of the equations (11) with finite energy condition modulo the gauge transformations $\mathcal{G}_{V}=\operatorname{Map}_{L_{2, l o c}^{2}}(V, U(1))$.

The flat connections on the determinant bundle, modulo the even gauge group $\mathcal{G}_{T^{2}}=\operatorname{Map}\left(T^{2}, U(1)\right)$, form a torus

$$
\chi\left(T^{2}\right)=H^{1}\left(T^{2}, \mathbb{R}\right) / 2 H^{1}\left(T^{2}, \mathbb{Z}\right),
$$

which is a $\mathbb{Z}_{2} \times \mathbb{Z}_{2}$ cover of the standard torus $\operatorname{Hom}\left(\pi_{1}\left(T^{2}\right), U(1)\right)=\mathbb{R}^{2} / \mathbb{Z}^{2}$. Let $\chi\left(T^{2}, V\right)$ be the moduli space of flat connections modulo the subgroup of the gauge transformations on $T^{2}$ which can be extended to $V$. Let $\pi$ denote the quotient map $\pi: \chi\left(T^{2}, V\right) \rightarrow \chi\left(T^{2}\right)$, which is a $\mathbb{Z}$-covering map.

Suppose we are given a smooth solution $(A, \psi)$ of the Seiberg-Witten equations, satisfying the finite energy condition (12). Then we will see that there is a choice of a connection $\tilde{A}$ in the gauge class of $A$ that approaches a flat connection on $T^{2}$, while the spinor $\psi$ vanishes in the limit on the cylindrical end. That is, if $s$ is the coordinate on $[0, \infty)$, we will show that $\lim _{s \rightarrow \infty}(\tilde{A}, \psi)=\left(a_{\infty}, 0\right)$ in the appropriate topology, for each finite energy solution $(A, \psi)$ to the Seiberg-Witten equations (11). Thus the asymptotic limit of the Seiberg-Witten monopoles on the manifold $V$ with a cylindrical end defines a boundary value map

$$
\mathcal{M}_{V} \stackrel{\partial_{\infty}}{\rightarrow} \chi\left(T^{2}, V\right) \stackrel{\pi}{\rightarrow} \chi\left(T^{2}\right) .
$$

We will show that, in a suitable topology, this boundary value map is well-defined and continuous. Then, we will describe the structure of the moduli space $\mathcal{M}_{V}$.

\subsection{Monopoles on $T^{2} \times[0, \infty)$}

We begin with the investigation of the behaviour of the solutions of the Seiberg-Witten equations on the cylindrical end $T^{2} \times[0, \infty)$. Fix a flat background connection $A_{0}$ on the determinant bundle $\operatorname{det}(W)$ with asymptotic limit $a_{0}$.

Lemma 3.1. Choose the coordinate $s \in[0, \infty)$ on the cylindrical end $T^{2} \times[0, \infty)$. Choose the $\mathrm{Spin}^{c}$ structure over $T^{2} \times[0, \infty)$ to be the pull-back of the $\mathrm{Spin}^{c}$ structure on $T^{2}$ with trivial determinant, induced by the complex structure. We can write $(A, \psi)$ as

$$
\left\{\begin{array}{l}
A=A_{0}+a(s)+h(s) d s \\
\psi=(\alpha(s), \beta(s)) \in \Lambda^{0,0} \oplus \Lambda^{0,1}=\Gamma(W) .
\end{array}\right.
$$


where $a(s)=a^{1,0}(s)+a^{0,1}(s) \in \Lambda^{1}\left(T^{2}, i \mathbb{R}\right), h(s) \in \Lambda^{0}\left(T^{2}, i \mathbb{R}\right)$. Then the Seiberg-Witten equations (11) can be written in the form

$$
\left\{\begin{array}{l}
F_{A_{0}+a}=\frac{i}{2}\left(|\alpha|^{2}-|\beta|^{2}\right) \omega \\
\frac{\partial a^{0,1}(s)}{\partial s}=i \bar{\alpha} \beta+\bar{\partial} h \\
\left(\begin{array}{cc}
i\left(\partial_{s}+h\right) & \bar{\partial}_{a(s)}^{*} \\
\bar{\partial}_{a(s)} & -i\left(\partial_{s}+h\right)
\end{array}\right)\left(\begin{array}{l}
\alpha \\
\beta
\end{array}\right)=0 .
\end{array}\right.
$$

where $\omega$ is the area 2-form on $T^{2}$ with $\int_{T^{2}} \omega=1$.

Proof. We may choose a trivialization of the cotangent bundle to $T^{2} \times[0, \infty)$ so that, using a full-stop to denote Clifford multiplication by a one form, we can make the identifications:

$$
d s .=\left(\begin{array}{rr}
i & 0 \\
0 & -i
\end{array}\right), \quad d x .=\left(\begin{array}{rr}
0 & -1 \\
1 & 0
\end{array}\right), \quad d y .=\left(\begin{array}{cc}
0 & i \\
i & 0
\end{array}\right)
$$

Letting the Hodge $*$ on forms on $T^{2} \times[0, \infty)$ be denoted by $*_{3}$, then under the preceding identifications we have

$$
\begin{aligned}
*_{3}(\sigma(\psi, \psi)) & =\frac{i}{2}\left(|\alpha|^{2}-|\beta|^{2}\right) \omega-i(\alpha \bar{\beta}+\bar{\alpha} \beta) \wedge d s \\
F_{A} & =F_{A_{0}+a}+\left(d_{T^{2}} h-\partial_{s} a\right) \wedge d s,
\end{aligned}
$$

hence we get

$$
\left\{\begin{array}{l}
F_{A_{0}+a}=\frac{i}{2}\left(|\alpha|^{2}-|\beta|^{2}\right) \omega \\
\frac{\partial a^{0,1}(s)}{\partial s}=i \bar{\alpha} \beta+\bar{\partial} h
\end{array}\right.
$$

The form $a(s) \in \Lambda^{1}\left(T^{2}, i \mathbb{R}\right)$ is uniquely determined, as an $i \mathbb{R}$-valued 1 -form, by its $(0,1)$ part $a^{0,1} \in \Lambda^{0,1}\left(T^{2}\right)$. Similarly, the Dirac operator on $T^{2} \times[0, \infty)$ can be expressed as

$$
\not \not_{a(s)+h(s) d s}=\left(\begin{array}{cc}
i\left(\partial_{s}+h\right) & \bar{\partial}_{a(s)}^{*} \\
\bar{\partial}_{a(s)} & -i\left(\partial_{s}+h\right)
\end{array}\right) .
$$

Thus gives the Dirac equation as in the Lemma. 
Let $(A, \psi)$ be an irreducible solution of the Seiberg-Witten equations on the manifold $V$. Along the cylindrical end $T^{2} \times[0, \infty)$ we can use Lemma 3.1 to write the Seiberg-Witten equations in the form

$$
\left\{\begin{array}{l}
\partial_{s} a^{0,1}=i \bar{\alpha} \beta+\bar{\partial} h \\
\partial_{s} \alpha=i \bar{\partial}_{a(s)}^{*} \beta-h \alpha, \\
\partial_{s} \beta=-i \bar{\partial}_{a(s)} \alpha-h \beta .
\end{array}\right.
$$

with the constraint $F_{a}=\frac{i}{2}\left(|\alpha|^{2}-|\beta|^{2}\right) \omega$. These equations are gauge-equivalent, through a gauge transformation in $\mathcal{G}_{V}$, to the following equations:

$$
\left\{\begin{array}{l}
\partial_{s} a^{0,1}=i \bar{\alpha} \beta \\
\partial_{s} \alpha=i \bar{\partial}_{a(s)}^{*} \beta \\
\partial_{s} \beta=-i \bar{\partial}_{a(s)} \alpha
\end{array}\right.
$$

on the configuration space $\mathcal{A}_{T^{2}}$ of triples $(a, \alpha, \beta)$, where $a$ is a $U(1)$-connection on $\operatorname{det}(W)$ and $(\alpha, \beta)$ is a section of $\operatorname{Spin}^{c}$ bundle $W$ over $T^{2}$. The following Lemma shows that (16) can be interpreted as a gradient flow equation.

Lemma 3.2. The equations (16) are the downward gradient flow equations of the $\mathcal{G}_{T^{2}}=\operatorname{Map}\left(T^{2}, U(1)\right)$-invariant functional

$$
f(a, \alpha, \beta)=-\int_{T^{2}}\left\langle\alpha, i \bar{\partial}_{a}^{*} \beta\right\rangle \omega
$$

on the space $\mathcal{A}_{T^{2}}$, where the product $\langle$,$\rangle denotes the natural inner product using the$ Hodge star operator.

Proof. Direct calculation shows that we have

$$
\nabla f(a, \alpha, \beta)=\left(-i \bar{\alpha} \beta-i \alpha \bar{\beta},-i \bar{\partial}_{a}^{*} \beta, i \bar{\partial}_{a} \alpha\right) .
$$

Critical points of the functional (17) with the condition $F_{a}=\frac{i}{2}\left(|\alpha|^{2}-|\beta|^{2}\right) \omega$ are all the elements $\left(a_{\infty}, 0,0\right)$, with $a_{\infty}$ a flat connection. This critical point set is denoted by $\chi\left(T^{2}\right)$, the quotient space of the flat connection by the even gauge transformation. If $(A(s), \psi(s))$ is a solution to the Seiberg-Witten equation on $[0, \infty) \times T^{2}$ in temporal gauge, then

$$
(A(s), \psi(s))=\left(A_{0}+a^{1,0}+a^{0,1},(\alpha, \beta)\right)
$$

satisfies the gradient flow equation of $f$ as given by (16).

The next few lemmata describe some fundamental properties about the solution to the Seiberg-Witten equation on the cylinder over $T^{2}$ in temporal gauge. 
Lemma 3.3. Let $\gamma(s)=(A(s), \psi(s))$ be a solution to the Seiberg-Witten equation on $\left[s_{1}, s_{2}\right] \times T^{2}$ in temporal gauge, then

$$
\int_{s_{1}}^{s_{2}}\|\nabla f(\gamma(s))\|_{L^{2}\left(T^{2}\right)}^{2} d s=\int_{\left[s_{1}, s_{2}\right] \times T^{2}}\left(\left|\nabla_{A} \psi\right|^{2}+\left|F_{A}\right|^{2}\right) d v o l .
$$

Proof. Since $(A(s), \psi(s))$ satisfies the Seiberg-Witten equation on $\left[s_{1}, s_{2}\right] \times T^{2}$ :

$$
\left\{\begin{array}{l}
\not \partial_{A} \psi=0 \\
* F_{A}=\sigma(\psi, \psi)
\end{array}\right.
$$

The Weitzenböck formula for the Dirac operator on $\left[s_{1}, s_{2}\right] \times T^{2}$ with flat metric then gives

$$
\not_{A}^{*} \not_{A} \psi=\nabla_{A}^{*} \nabla_{A} \psi-\frac{1}{2}\left(* F_{A}\right) \cdot \psi=0 .
$$

Take the inner product of both sides with $\psi$, use the Seiberg-Witten equation, and note that $\left\langle\left(-* F_{A}\right) \cdot \psi, \psi\right\rangle=2\left\langle * F_{A}, \sigma(\psi, \psi)\right\rangle=2\left|F_{A}\right|^{2}$. We obtain

$$
\frac{1}{2} d^{*} d|\psi|^{2}+\left|\nabla_{A} \psi\right|^{2}+\left|F_{A}\right|^{2}=0
$$

Integrating the above identity over $\left[s_{1}, s_{2}\right] \times T^{2}$, we can write the result as

$$
\begin{aligned}
& \int_{\left[s_{1}, s_{2}\right] \times T^{2}}\left(\left|\nabla_{A} \psi\right|^{2}+\left|F_{A}\right|^{2}\right) d v o l \\
= & -\frac{1}{2} \int_{\left[s_{1}, s_{2}\right] \times T^{2}} d * d|\psi|^{2} \\
= & -\frac{1}{2} \int_{\partial\left(\left[s_{1}, s_{2}\right] \times T^{2}\right)}\left(\partial_{s}\langle\psi, \psi\rangle\right) \omega \\
= & \int_{T^{2}}\left\langle\alpha\left(s_{1}\right), i \bar{\partial}_{A\left(s_{1}\right)}^{*} \beta\left(s_{1}\right)\right\rangle \omega-\int_{T^{2}}\left\langle\alpha\left(s_{2}\right), i \bar{\partial}_{A\left(s_{2}\right)}^{*} \beta\left(s_{2}\right)\right\rangle \omega .
\end{aligned}
$$

Here we write $\psi(s)=(\alpha(s), \beta(s))$ as a spinor on $T^{2}$ and use the equation (16) for $\partial_{s} \psi$. Note that $\gamma(s)=(A(s), \alpha(s), \beta(s))$ solves the gradient flow equation of $f$, hence

$$
\begin{aligned}
& \int_{s_{1}}^{s_{2}}\|\nabla f(\gamma(s))\|_{L^{2}\left(T^{2}\right)}^{2} d s \\
= & \int_{T^{2}}\left\langle\alpha\left(s_{1}\right), i \bar{\partial}_{A\left(s_{1}\right)}^{*} \beta\left(s_{1}\right)\right\rangle \omega-\int_{T^{2}}\left\langle\alpha\left(s_{2}\right), i \bar{\partial}_{A\left(s_{2}\right)}^{*} \beta\left(s_{2}\right)\right\rangle \omega \\
= & \int_{\left[s_{1}, s_{2}\right] \times T^{2}}\left(\left|\nabla_{A} \psi\right|^{2}+\left|F_{A}\right|^{2}\right) d v o l .
\end{aligned}
$$


Lemma 3.4. Let $\gamma(s)=(A(s), \psi(s))$ be a solution to the Seiberg-Witten equation on $N=[t-1, t+1] \times T^{2}$ in temporal gauge for any $t \in[0, \infty)$. If $\int_{N}\left|F_{A}\right|^{2} d v o l=E_{N}$, then there exists a constant $C_{0}$ such that the following estimates hold

$$
\begin{gathered}
\int_{\left[t-\frac{1}{2}, t+\frac{1}{2}\right] \times T^{2}}\left|\nabla_{A} \psi\right|^{2} d v o l \leq C_{0} \sqrt{E_{N}} \\
\int_{t-\frac{1}{2}}^{t+\frac{1}{2}}\|\nabla f(\gamma(s))\|_{L^{2}}^{2} d s \leq C_{0} \sqrt{E_{N}}+E_{N} .
\end{gathered}
$$

Moreover, if $(A(s), \psi(s))$ is a solution to the Seiberg-Witten equation on $[-1, \infty) \times T^{2}$ in temporal gauge with finite energy, then the corresponding flowline on $\mathcal{A}_{T^{2}}$ of $f$ has finite variation of $f$ along $[0, \infty) \times T^{2}$.

Proof. From the $L^{2}$-bound on $\left|F_{A}\right|$, we immediately obtain a $L^{4}$-bound on $\psi$ from the Seiberg-Witten equation,

$$
\|\psi\|_{L^{4}(N)}^{4}=\int_{N}|\psi|^{4} d v o l=\frac{1}{4} \int_{N}\left|F_{A}\right|^{2} d v o l .
$$

By the Cauchy-Schwartz inequality, we get

$$
\|\psi\|_{L^{2}(N)}^{2} \leq \sqrt{\operatorname{Vol}(N)}\|\psi\|_{L^{4}(N)}^{2}=\frac{\sqrt{2}}{2}\left\|F_{A}\right\|_{L^{2}(N)} .
$$

Here we use that $\operatorname{Vol}(N)=2$. In the proof the previous lemma, we found that $\psi$ satisfies

$$
\frac{1}{2} d^{*} d|\psi|^{2}+\left|\nabla_{A} \psi\right|^{2}+\left|F_{A}\right|^{2}=0
$$

Multiplying both sides of the above equation with a cut-off function $\rho$ which equals 1 on $\left[t-\frac{1}{2}, t+\frac{1}{2}\right] \times T^{2}$ and vanishes near the boundary of $N$, and then integrating by parts, we obtain

$$
\left\|\nabla_{A} \psi\right\|_{L^{2}\left(\left[t-\frac{1}{2}, t+\frac{1}{2}\right] \times T^{2}\right)}^{2} \leq-\frac{1}{2} \int_{N} d^{*} d|\psi|^{2} \rho d v o l \leq C_{1}\|\psi\|_{L^{2}(N)}^{2} \leq \frac{\sqrt{2}}{2} C_{1}\left\|F_{A}\right\|_{L^{2}(N)},
$$

where $C_{1}$ is a constant depending only on the cut-off function $\rho$. Putting the above inequalities together we get the estimates as claimed with $C_{0}=\frac{\sqrt{2}}{2} C_{1}$.

The finite variation of $f$ along $[0, \infty) \times T^{2}$ for a solution on $[-1, \infty) \times T^{2}$ is the direct consequence of adding up over a sequence of middle tubes of length 2 , namely, $\left\{[i-1, i+1] \times T^{2} \mid i=0,1,2, \cdots\right\}$, hence

$$
\begin{aligned}
& \int_{0}^{\infty}\|\nabla f(\gamma(s))\|_{L^{2}\left(T^{2}\right)}^{2} d s \\
\leq & 2 \int_{-1}^{\infty}\left\|F_{A}\right\|_{L^{2}\left(T^{2}\right)}^{2} d s+2 C_{0} \sqrt{\int_{-1}^{\infty}\left\|F_{A}\right\|_{L^{2}\left(T^{2}\right)}^{2} d s}<\infty .
\end{aligned}
$$


Lemma 3.5. Let $(a(s), \alpha(s), \beta(s))$ be a monopole on $T^{2} \times[0, \infty)$ with finite energy

$$
\int_{T^{2} \times[0, \infty)}|F|^{2} d v o l<\infty
$$

Then there exists a sequence $\left\{s_{n}\right\}$ such that $\lim _{s_{n} \rightarrow \infty}\left(a\left(s_{n}\right), \alpha\left(s_{n}\right), \beta\left(s_{n}\right)\right)$ exists and represents a point in $\chi\left(T^{2}\right)$.

Proof. Write the curvature of $a(s)$ on $T^{2} \times[0, \infty)$ as $F_{a(s)}-\partial_{s}(a(s)) \wedge d s$, where $F_{a(s)}$ is the curvature on $T^{2}$. Then we have the following calculation:

$$
\begin{aligned}
& \int_{T^{2} \times[0, \infty)}|F|^{2} d v o l \\
= & \int_{T^{2} \times[0, \infty)}\left(\left|F_{a(s)}\right|^{2}+\left|\partial_{s}(a(s))\right|^{2}\right) d v o l,
\end{aligned}
$$

which implies that as $s \rightarrow \infty$,

$$
\left|F_{a(s)}\right| \rightarrow 0, \quad\left|\partial_{s}(a(s))\right| \rightarrow 0
$$

By Uhlenbeck's weak compactness result and the compactness of $\chi\left(T^{2}\right)$, we know that $a(s)$ weakly converges to a flat connection.

By the monopole equation on $T^{2} \times[0, \infty)$, we also obtain

$$
|\alpha(s)|^{2}-|\beta(s)|^{2} \rightarrow 0, \quad \bar{\alpha}(s) \beta(s) \rightarrow 0 .
$$

This implies that $[a(s), \alpha(s), \beta(s)]$ converges weakly to a point in $\chi\left(T^{2}\right)$.

To establish strong convergence to a point in $\chi\left(T^{2}\right)$ for any finite energy monopole on $[0, \infty) \times T^{2}$, we need to apply L. Simon's type result of "small energy implying small length" as in [23]. We will address this issue at the end of this subsection.

Let $\mathcal{G}_{T^{2}}^{0}$ be the based gauge group on $\operatorname{det}(W)$, that is, those gauge transformations which equal the identity at a fixed based point. Denote by $\mathcal{B}_{T^{2}}^{0}$ the quotient space of $\mathcal{A}_{T^{2}}$ by the free action of $\mathcal{G}_{T^{2}}^{0}$. Note that the gradient flow of $f$ preserves the constraint $F_{a}=\frac{i}{2}\left(|\alpha|^{2}-\left|\beta^{2}\right|\right) \omega$, hence we can consider gradient flow lines of $f$ restricted to

$$
\mathcal{C}_{T^{2}}=\left\{(a, \alpha, \beta) \mid F_{a}=\frac{i}{2}\left(|\alpha|^{2}-\left|\beta^{2}\right|\right) \omega\right\} / \mathcal{G}_{T^{2}}^{0}
$$

as a subset of $\mathcal{B}_{T^{2}}^{0}$.

The space $\mathcal{C}_{T^{2}}$ is a singular space, the singular set consisting of $[a, \alpha, \beta]$ where $a$ is a flat connection and $(\alpha, \beta)$ is a spinor section satisfying the pointwise condition $|\alpha|=|\beta|$. We want to study the asymptotic behavior of the finite energy monopole on $T^{2} \times[0, \infty)$, that is, the asymptotic behavior of the gradient flow of $f$ restricted to $\mathcal{C}_{T^{2}}$. 
If we consider a neighborhood of $\mathcal{C}_{T^{2}}$ in the whole configuration space $\mathcal{B}_{T^{2}}^{0}$, this introduces new critical points which consist of the $[\Theta, \alpha, \beta]$, with $\Theta \in \chi\left(T^{2}\right)$ and $(\alpha, \beta)$ satisfying

$$
\bar{\alpha} \beta=\bar{\partial}_{\Theta}(\alpha)=\bar{\partial}_{\Theta}^{*}(\beta)=0 .
$$

Note that there is a unique point $\Theta \in \chi\left(T^{2}\right)$ with $\operatorname{ker}\left(\bar{\partial}_{\Theta}+\bar{\partial}_{\Theta}^{*}\right)$ non-trivial, which satisfies

$$
\operatorname{Ker} \bar{\partial}_{\Theta} \cong K e r \bar{\partial}_{\Theta}^{*} \cong \mathbb{C} .
$$

Since we are only interested in the behavior of the monopoles on $T^{2} \times[0, \infty)$, among flowlines of $f$ on $\mathcal{B}_{T^{2}}^{0}$, we only study those that flow to the critical manifold $\chi\left(T^{2}\right)$. The Hessian operator of $f$ at the critical point $\left[a_{\infty}, 0,0\right]$ in $\chi\left(T^{2}\right)$ is given by

$$
Q_{\left[a_{\infty}, 0,0\right]}\left(a_{1}, \alpha_{1}, \beta_{1}\right)=\left(0,-i \bar{\partial}_{a_{\infty}}^{*} \beta_{1}, i \bar{\partial}_{a_{\infty}} \alpha_{1}\right) .
$$

where $\left(a_{1}, \alpha_{1}, \beta_{1}\right)$ is a $L_{1}^{2}$-tangent vector of $\mathcal{B}_{T^{2}}^{0}$ at $\left[a_{\infty}, 0,0\right]$, that is, $\left(a_{1}, \alpha_{1}, \beta_{1}\right)$ satisfies the condition $d^{*} a_{1}=0$, and we view $\left(0,-i \bar{\partial}_{a_{\infty}}^{*} \beta_{1}, i \bar{\partial}_{a_{\infty}} \alpha_{1}\right)$ as a $L^{2}$-tangent vector of $\mathcal{B}_{T^{2}}^{0}$ at $\left[a_{\infty}, 0,0\right]$. Then the following lemma is obtained by a direct calculation.

Lemma 3.6. For $a_{\infty} \neq \Theta$ in $\chi\left(T^{2}\right), f$ is non-degenerate at $a_{\infty}$ in the sense of MorseBott, that is, the Hessian operator $Q$ at $\left[a_{\infty}, 0,0\right]$ is non-degenerate in the normal direction to the critical manifold in the tangent space of $\mathcal{B}_{T^{2}}^{0}$ at $\left[a_{\infty}, 0,0\right]$. At the point $\Theta$, the kernel of the Hessian operator is given by

$$
H^{1}\left(T^{2}, i \mathbb{R}\right) \oplus \operatorname{Ker} \bar{\partial}_{\Theta} \oplus \operatorname{Ker} \bar{\partial}_{\Theta}^{*} \cong \mathbb{C}^{3} .
$$

Let $U_{\Theta}$ be a small open neighbourhood of $\Theta$ in $\chi\left(T^{2}\right)$. For any point $a_{\infty} \in \chi\left(T^{2}\right) \backslash U_{\Theta}$, the spectrum of $Q_{a_{\infty}}=Q_{\left[a_{\infty}, 0,0\right]}$ (as a first order elliptic operator (18)) is discrete, real and without accumulation points. Let $\mu_{a_{\infty}}>0$ be the smallest absolute value of the non-zero eigenvalues of the Hessian operator $Q_{a_{\infty}}$. Now we can establish the decay estimate for the Seiberg-Witten monopoles along the cylindrical end of $V$. The first exponential decay estimate is for a solution to the Seiberg-Witten equation on $[0, R] \times T^{2}(R>1)$ which is near a critical point in $\chi\left(T^{2}\right)$.

Lemma 3.7. Suppose that $x(s)=[a(s), \psi(s)]$ is a flow line of $f$, corresponding to an irreducible finite energy monopole on $T^{2} \times[0, R]$ in temporal gauge. There is a representative $(A(s), \psi(s))$ which is gauge equivalent to $\left(a_{\infty}, 0\right)+(b, \eta)$, where $\left[a_{\infty}, 0\right] \neq \Theta$. There exist positive constants $\epsilon, \delta, C_{1}$ such that, if $(b, \eta)$ has $L_{1}^{2}$-norm less than $\epsilon$ on any s-slice, then

$$
\|(b(s), \eta(s))\|_{L_{1}^{2}\left(T^{2}\right)} \leq C_{1}(\exp (-\delta s)+\exp (-\delta(R-s)))
$$

on any constant $s$-slice $(s \in[0, R])$. 
Proof. Write $\lambda=(b, \eta)$, then $\lambda$ satisfies the following equation:

$$
\partial_{s} \lambda=Q_{a_{\infty}} \lambda+n(\lambda),
$$

Here $n(\lambda)$ is second order in $\lambda$ with $\|n(\lambda)\|_{L^{2}\left(T^{2}\right)} \leq \epsilon\|\lambda\|_{L^{2}\left(T^{2}\right)}$ and $Q_{a_{\infty}}=Q_{\left[a_{\infty}, 0,0\right]}$. Note that the flowline of $f$ on $\chi\left(T^{2}\right)$ is static, hence we can establish the analogous result as Lemma 5.4.1 in [23] as follows.

Let $\lambda_{ \pm}$denote the projection of $\lambda$ onto the eigenspaces of $Q_{a_{\infty}}$ with positive and negative eigenvalues. Let $\left\|\lambda_{ \pm}\right\|$be the functions on $[0, R]$ given by the $L^{2}\left(T^{2}\right)$-norm on the $s$-slice of $[0, R] \times T^{2}$. Then we have

$$
\begin{aligned}
& \partial_{s}\left\|\lambda_{+}\right\|-\left(\mu_{a_{\infty}}-\epsilon\right)\left\|\lambda_{+}\right\|+\epsilon\left\|\lambda_{-}\right\| \geq 0 \\
& \partial_{s}\left\|\lambda_{-}\right\|+\left(\mu_{a_{\infty}}-\epsilon\right)\left\|\lambda_{-}\right\|-\epsilon\left\|\lambda_{+}\right\| \leq 0 .
\end{aligned}
$$

When $\epsilon<<\mu_{a_{\infty}}$, from the above inequalities together with the comparison principle (Cf. Lemma 9.4 [32]), we obtain that the $L^{2}$-norm of $\lambda$ on the $s$-slice is decaying exponentially with decay rate $\delta \leq \mu_{a_{\infty}} / 2$. Then the claim of the lemma follows from the standard bootstrapping argument.

Proposition 3.8. Suppose that $\gamma(s)=[a(s), \psi(s)]$ is an irreducible flow line of $f$, corresponding to an irreducible finite energy monopole on $T^{2} \times[0, \infty)$, with asymptotic limit $\left[a_{\infty}, 0,0\right]$ where $\left[a_{\infty}\right] \neq \Theta \in \chi\left(T^{2}\right)$. Then, there exist gauge representatives $(a(s), \psi(s))$ for $\gamma(s)$ and $a_{\infty}$ for $\left[a_{\infty}, 0,0\right]$ such that $\left(a(s)-a_{\infty}, \psi(s)\right)$ decays exponentially along with its first derivative as $s \rightarrow \infty$.

Proof. From Lemma 3.4, we know that the variation of $f$ is finite, that is,

$$
\int_{1}^{\infty}\|\nabla f(\gamma(s))\|_{L^{2}\left(T^{2}\right)}^{2} d s
$$

is finite. Then we have the following estimate, whose proof is analogous to the proof of Lemma 6.14 of [24]. We sketch the proof here.

Claim: There exist constants $E_{0}$ and $C$ such that for any $R>1$, and for $\gamma(s)=(A(s), \psi(s))$ any solution to the Seiberg-Witten equation in temporal gauge on $[0, R+1] \times T^{2}$ satisfies

$$
\int_{0}^{R+1}\|\nabla f(\gamma(s))\|_{L^{2}\left(T^{2}\right)}^{2} d s \leq E_{0}
$$

then we have the estimate

$$
\int_{1}^{R}\|\nabla f(\gamma(s))\|_{L_{1}^{2}\left(T^{2}\right)}^{2} d s \leq C \int_{0}^{R+1}\|\nabla f(\gamma(s))\|_{L^{2}\left(T^{2}\right)}^{2} d s .
$$


Proof of the Claim Let $\gamma(s)=((A(s), \psi(s))$ be a solution to the Seiberg-Witten equation on $N=\left[s_{1}, s_{2}\right] \times T^{2}$ in temporal gauge, then from Lemma 3.3, we have

$$
\int_{s_{1}}^{s_{2}}\|\nabla f(\gamma(s))\|_{L^{2}\left(T^{2}\right)}^{2} d s=\int_{\left[s_{1}, s_{2}\right] \times T^{2}}\left(\left|\nabla_{A} \psi\right|^{2}+\left|F_{A}\right|^{2}\right) d v o l .
$$

Denote by

$$
\begin{aligned}
E & =\int_{s_{1}}^{s_{2}} \| \nabla f\left(\gamma(s) \|_{L^{2}\left(T^{2}\right)}^{2} d s\right. \\
& =\int_{N}\left(\left|\partial_{s} A\right|^{2}+\left|\partial_{s} \psi\right|^{2}\right) d v o l .
\end{aligned}
$$

Then we have the following estimates

$$
\left\|F_{A}\right\|_{L^{2}(N)} \leq \sqrt{E} ; \quad\|\psi\|_{L^{4}(N)}^{2}=2\left\|F_{A}\right\|_{L^{2}(N)} \leq 2 \sqrt{E} .
$$

We proceed as in Lemma 6.14 of [24] and differentiate the Seiberg-Witten equations to get

$$
\begin{gathered}
d \partial_{s} A=* \sigma\left(\partial_{s} \psi, \psi\right) \\
\not \partial_{A} \partial_{s} \psi+\left(\partial_{s} A\right) \cdot \psi=0 .
\end{gathered}
$$

The gauge fixing condition implies that

$$
d^{*}\left(\partial_{s} A\right)+i \operatorname{Im}\left\langle\partial_{s} \psi, \psi\right\rangle=0
$$

Introduce a cutoff function $\rho$ identically equal to 1 on the middle third piece of $N$ and vanishes near the boundary such that $|d \rho|$ is at most $\frac{M}{s_{2}-s_{1}}$ where $M$ is a universal constant. Set $(V, \lambda)=\left(\rho \partial_{s} A, \rho \partial_{s} \psi\right)$. Then we can estimate the quantity

$$
S W(V, \lambda)=\left(d V-* \sigma(\lambda, \psi), \not \partial_{A}(\lambda)+V \cdot \psi, d^{*} V+i \operatorname{Im}\langle\lambda, \psi\rangle\right)
$$

by

$$
\|S W(V, \lambda)\|_{L^{2}(N)}^{2} \leq \frac{C}{\left(s_{2}-s_{1}\right)^{2}}\left(\left\|\partial_{s} A\right\|_{L^{2}(N)}^{2}+\left\|\partial_{s} \psi\right\|_{L^{2}(N)}^{2}\right) .
$$

Here $C$ is a universal constant depending only on $\rho$.

On the other hand, we can estimate

$$
\begin{aligned}
\|S W(V, \lambda)\|_{L^{2}(N)}^{2} \geq & \frac{1}{2}\left(\|d V\|_{L^{2}(N)}^{2}+\left\|\not \partial_{A}(\lambda)\right\|_{L^{2}(N)}^{2}+\left\|d^{*} V\right\|_{L^{2}(N)}^{2}\right) \\
& -2\left(\|\sigma(\lambda, \psi)\|_{L^{2}(N)}^{2}+\|V \cdot \psi\|_{L^{2}(N)}^{2}\|\operatorname{Im}\langle\lambda, \psi\rangle\|_{L^{2}(N)}^{2}\right) .
\end{aligned}
$$


Assume that $l=s_{2}-s_{1} \leq 1$, then the Sobolev multiplication theorem and Sobolev embedding theorem imply that there are constants $C_{0}$ and $C_{1}$ such that

$$
\begin{aligned}
\|\sigma(\lambda, \psi)\|_{L^{2}(N)}^{2} & \leq C_{0}\|\lambda\|_{L^{4}(N)}^{2}\|\psi\|_{L^{4}(N)}^{2} \\
& \leq C_{1} \sqrt{E}\|\lambda\|_{L_{1}^{2}(N)}^{2} .
\end{aligned}
$$

Similarly, by choosing $C_{1}$ appropriately, we have

$$
\begin{gathered}
\|V \cdot \psi\|_{L^{2}(N)}^{2} \leq C_{1} \sqrt{E}\|V\|_{L_{1}^{2}(N)}^{2} \\
\|\operatorname{Im}\langle\lambda, \psi\rangle\|_{L^{2}(N)}^{2} \leq C_{1} \sqrt{E}\|\lambda\|_{L_{1}^{2}(N)}^{2} .
\end{gathered}
$$

These inequalities imply that

$$
\begin{aligned}
& \|d V\|_{L^{2}(N)}^{2}+\left\|\not \partial_{A}(\lambda)\right\|_{L^{2}(N)}^{2}+\left\|d^{*} V\right\|_{L^{2}(N)}^{2} \\
\leq & 8 C_{1} \sqrt{E}\left(\|V\|_{L_{1}^{2}(N)}^{2}+\|\lambda\|_{L_{1}^{2}(N)}^{2}\right)+2\|S W(V, \lambda)\|_{L^{2}(N)}^{2}
\end{aligned}
$$

Standard estimate for the elliptic operator $\left(d+d^{*}, \not_{A}\right)$ can be employed to show that there is a constant $C_{2}$ such that

$$
\begin{aligned}
\|V\|_{L_{1}^{2}(N)}^{2}+\|\lambda\|_{L_{1}^{2}(N)}^{2} \leq & C_{2}\left(\|d V\|_{L^{2}(N)}^{2}+\left\|\not \partial_{A}(\lambda)\right\|_{L^{2}(N)}^{2}+\left\|d^{*} V\right\|_{L^{2}(N)}^{2}\right) \\
& +C_{2}\left(\|V\|_{L^{2}(N)}^{2}+\|\lambda\|_{L^{2}(N)}^{2}\right) .
\end{aligned}
$$

The Cauchy-Schwartz inequality and the Sobolev embedding theorem imply that there exists a constant $C_{3}$ such that

$$
\left(\|V\|_{L^{2}(N)}^{2}+\|\lambda\|_{L^{2}(N)}^{2}\right) \leq C_{3} \sqrt{s_{2}-s_{1}}\left(\|V\|_{L_{1}^{2}(N)}^{2}+\|\lambda\|_{L_{1}^{2}(N)}^{2}\right) .
$$

Put all these inequalities together, we have

$$
\begin{aligned}
\|V\|_{L_{1}^{2}(N)}^{2}+\|\lambda\|_{L_{1}^{2}(N)}^{2} \leq & \frac{2 C C_{2}}{\left(s_{2}-s_{1}\right)^{2}}\left(\left\|\partial_{s} A\right\|_{L^{2}(N)}^{2}+\partial_{s} \psi \|_{L^{2}(N)}^{2}\right) \\
& +8 C_{1} C_{2} \sqrt{E}\left(\|V\|_{L_{1}^{2}(N)}^{2}+\|\lambda\|_{L_{1}^{2}(N)}^{2}\right) \\
& +C_{2} C_{3} \sqrt{s_{2}-s_{1}}\left(\|V\|_{L_{1}^{2}(N)}^{2}+\|\lambda\|_{L_{1}^{2}(N)}^{2}\right)
\end{aligned}
$$

Then there is a constant $E_{0}$ and a constant $l_{0}$ satisfying

$$
1-8 C_{1} C_{2} \sqrt{E_{0}}-C_{2} C_{3} \sqrt{l_{0}} \geq \frac{1}{2}
$$

such that if $E \leq E_{0}$ and $s_{2}-s_{1}=l_{0}$, there is a constant $C_{4}$ with the following estimate

$$
\|V\|_{L_{1}^{2}(N)}^{2}+\|\lambda\|_{L_{1}^{2}(N)}^{2} \leq \frac{C_{4}}{l_{0}^{2}}\left(\left\|\partial_{s} A\right\|_{L^{2}(N)}^{2}+\left\|\partial_{s} \psi\right\|_{L^{2}(N)}^{2}\right) .
$$


Since on the middle third piece $N^{\prime},\left.V\right|_{N^{\prime}}=\left.\partial_{s} A\right|_{N^{\prime}}$ and $\left.\lambda\right|_{N^{\prime}}=\left.\partial_{s} \psi\right|_{N^{\prime}}$, this implies that for any tube $N=\left[s_{1}, s_{2}\right] \times T^{2}$ of length $l_{0}$ and any solution $(A(s), \psi(s))$ on $N$ of energy at most $E_{0}$, we have

$$
\left\|\partial_{s} A\right\|_{L_{1}^{2}\left(N^{\prime}\right)}^{2}+\left\|\partial_{s} \psi\right\|_{L_{1}^{2}\left(N^{\prime}\right)}^{2} \leq \frac{C_{4}}{l_{0}^{2}}\left(\left\|\partial_{s} A\right\|_{L^{2}(N)}^{2}+\left\|\partial_{s} \psi\right\|_{L^{2}(N)}^{2}\right) .
$$

Then the estimate in the claim follows by adding up a sequence of middle third pieces of tubes (length $l_{0}$ ) with the constant $C=3 C_{4} / l_{0}^{2}$ and $E_{0}$ as above.

With this claim and Lemma 3.7, we can prove Proposition 3.8 using the method of the proof of Proposition 6.16 [24] and the fact that $f$ is a Morse-Bott function on $\mathcal{B}_{T^{2}}^{0}$ and satisfies the Palais-Smale condition on paths coming from monopoles on $[0, \infty) \times T^{2}$.

Since $\chi\left(T^{2}\right) \backslash U_{\Theta}$ is compact, we can set $\delta=\frac{1}{2} \min \left\{\mu_{a_{\infty}} \mid a_{\infty} \in \chi\left(T^{2}\right) \backslash U_{\Theta}\right\}$. Then, when restricted to the cylindrical end, any Seiberg-Witten monopole on $V$ with finite energy and with asymptotic limit in $\chi\left(T^{2}\right) \backslash U_{\Theta}$ has an exponential decay at a rate at least $\delta$.

In order to prove that the boundary value map (13) is well-defined and continuous, we need to resort to the "finite energy implies finite length" principle of L. Simon([30] (see Corollary 4.2.5 in [23]).

Remark 3.9. Given that $f$ is a real analytic function, the work of L. Simon as explained in [23] can be employed to prove a more general Eojasiewicz inequality for $f$ at any critical point in $\chi\left(T^{2}\right)$. Let $\gamma(s)$ be a flow line of $f$, corresponding to an irreducible finite energy solution of the Seiberg-Witten equations on $T^{2} \times[0, \infty)$. Then, there exist constants $0<b \leq 1$ and $0<c \leq \frac{1}{2}$ such that, when $s>R>>1$, we have

$$
\begin{aligned}
& \inf _{a_{\infty} \in \chi\left(T^{2}\right)}\left\|\gamma(s)-a_{\infty}\right\|_{L^{2}} \leq\left(\|\nabla f(x(s))\|_{L^{2}}\right)^{b}, \\
& |f(\gamma(s))|^{1-c} \leq\|\nabla f(\gamma(s))\|_{L^{2}} .
\end{aligned}
$$

At the smooth critical points in $\chi\left(T^{2}\right)$, the Eojasiewicz inequalities have the best exponents $b=1$ and $c=\frac{1}{2}$. The direct consequence of these Eojasiewicz inequalities is the following finite length result for flow lines:

$$
\int_{s_{1}}^{s_{2}}\left\|\frac{\partial \gamma(s)}{\partial s}\right\|_{L^{2}} d s \leq \frac{4}{c}\left|f\left(\gamma\left(s_{1}\right)\right)-f\left(\gamma\left(s_{2}\right)\right)\right|^{c} .
$$

Now we have a setting analogous to the key results in [23] (pages 60-70) in our situation. The arguments in [23], adapt to the present context, hence imply that the boundary value map (13) is well-defined and continuous as a map

$$
\partial_{\infty}: \quad \mathcal{M}_{V}^{*} \rightarrow \chi\left(T^{2}\right),
$$


In the next subsection, we will study the local properties of the map $\partial_{\infty}$ around the singular point $\Theta$ and the structure of $\mathcal{M}_{V}^{*}$. We remind the reader that we have established the exponential decay property of the monopoles in $\mathcal{M}_{V}^{*}$ with asymptotic limits away from $\Theta$.

\subsection{Local structure of moduli space of irreducible monopoles}

Let $U_{\Theta}$ be a small neighbourhood of $\Theta$ in $\chi\left(T^{2}\right)$. In this section, we will study the local structure of the moduli spaces $\mathcal{M}_{V}^{*} \backslash\left(\bar{\partial}_{\infty}\right)^{-1}\left(U_{\Theta}\right)$ and $\left(\bar{\partial}_{\infty}\right)^{-1}\left(U_{\Theta}\right)$. Here $\bar{\partial}_{\infty}$ is the composition of $\partial_{\infty}$ and $\pi$ in (13).

For the structure of the moduli space $\mathcal{M}_{V}^{*} \backslash\left(\bar{\partial}_{\infty}\right)^{-1}\left(U_{\Theta}\right)$, the exponential decay property implies that we can introduce weighted Sobolev norms in order to study the Fredholm theory of the linearization of the equations. With $\delta$ as in the previous subsection, we define the space

$$
\mathcal{A}_{V, T^{2}}=\left\{\begin{array}{l|l}
(A, \psi) \mid \begin{array}{l}
(1) A \text { is an extended } L_{2, \delta^{-}}^{2} \text { connection on } \operatorname{det}(W) \\
(2) \psi \text { is an } L_{2, \delta^{-}}^{2} \text {-spinor on } W
\end{array}
\end{array}\right\},
$$

where extended $L_{2, \delta}^{2}$-connection means that there exists an imaginary-valued harmonic 1-form $A_{\infty}$ in $H^{1}\left(T^{2}, i \mathbb{R}\right)$ such that $A-A_{\infty}$ is an $L_{2, \delta}^{2}$-connection on $\operatorname{det}(W)$, where $L_{2, \delta}^{2}$ denotes the Sobolev norm with weight as in [17]. To be precise, we choose the weight

function $e_{\delta}(t)=e^{\tilde{\delta}(t) / 2}$, where $\tilde{\delta}(t)$ is a smooth function with bounded derivatives, such that $\tilde{\delta}(t) \equiv-\delta t$ for $t \leq-1$ and $\tilde{\delta}(t) \equiv \delta t$ for $t \geq 1$, and for some fixed positive number $\delta$ defined as

$$
\delta=\frac{1}{2} \min \left\{\mu_{a_{\infty}} \mid a_{\infty} \in \chi\left(T^{2}\right) \backslash U_{\Theta}\right\} .
$$

The $L_{k, \delta}^{2}$ norm is defined as $\|f\|_{2, k, \delta}=\left\|e_{\delta} f\right\|_{2, k}$. The weight $e_{\delta}$ imposes an exponential decay as an asymptotic condition along the cylinder. We define the gauge group $\mathcal{G}_{V, T^{2}}$ to be the $L_{3, l o c}^{2}$-gauge transformations such that there exists $g_{\infty} \in U(1)$ with $g_{\infty}^{-1} g-1$ an $L_{3, \delta}^{2}$-gauge transformation.

Assume that $x=(A, \psi) \in \mathcal{A}_{V, T^{2}}$ is an irreducible $(\psi \neq 0)$ perturbed Seiberg-Witten monopole on $V$ with finite energy, where the perturbation is in the form of Section 2 with compact support. Then from the results of previous subsection, we can assume further that $A_{\infty}$ represents a flat connection $a_{\infty}$ in $\chi\left(T^{2}\right) \backslash U_{\Theta}$. Then the irreducible part of the fiber $\left(\bar{\partial}_{\infty}\right)^{-1}\left(a_{\infty}\right)$ has a deformation complex

$$
0 \rightarrow \Lambda_{L_{3, \delta}^{2}}^{0}(V, i \mathbb{R}) \stackrel{G}{\rightarrow} \Lambda_{L_{2, \delta}^{2}}^{1}(V, i \mathbb{R}) \oplus L_{2, \delta}^{2}(W) \stackrel{L}{\rightarrow} \Lambda_{L_{1, \delta}^{2}}^{1}(V, i \mathbb{R}) \oplus L_{1, \delta}^{2}(W)
$$

where $G$ is the map which gives the infinitesimal gauge transformations:

$$
\left.G\right|_{(A, \psi)}(f)=(-d f, f \psi)
$$


and $L$ is the linearization

$$
L_{A, \psi}(\alpha, \phi)=\left\{\begin{array}{c}
* d \alpha-\sigma(\psi, \phi) \\
\not_{A} \phi+\frac{1}{2} \alpha . \psi
\end{array}\right.
$$

of the perturbed Seiberg-Witten equations (11) on $V$. We can assemble the deformation complex (22) into the following operator:

$$
\left(G_{\delta}^{*}, L\right): \quad \Lambda_{L_{2, \delta}^{2}}^{1}(V, i \mathbb{R}) \oplus L_{2, \delta}^{2}(W) \rightarrow \Lambda_{L_{1, \delta}^{2}}^{0}(V, i \mathbb{R}) \oplus \Lambda_{L_{1, \delta}^{2}}^{1}(V, i \mathbb{R}) \oplus L_{1, \delta}^{2}(W)
$$

where $\left(G_{\delta}^{*}, L\right)(\alpha, \phi)$ is given by

$$
\left(e_{\delta}^{-1} d^{*}\left(e_{\delta} \alpha\right)+i \operatorname{Im}\langle\psi, \phi\rangle, * d \alpha-\sigma(\psi, \phi), \not_{A} \phi+\frac{1}{2} \alpha . \psi\right) .
$$

With the choice of $e_{\delta}$ as in the previous section, $\left(G_{\delta}^{*}, L\right)$ is a Fredholm operator of index 0 .

The deformation complex for the moduli space $\mathcal{M}_{V} \backslash\left(\bar{\partial}_{\infty}\right)^{-1}\left(U_{\Theta}\right)$ is given by

$$
0 \rightarrow T_{i d} \mathcal{G}_{V, T^{2}} \stackrel{G}{\rightarrow} T_{x} \mathcal{A}_{V, T^{2}} \stackrel{L}{\rightarrow} \Lambda_{L_{1, \delta}^{2}}^{1}(V, i \mathbb{R}) \oplus L_{1, \delta}^{2}(W) .
$$

These two complexes are related by the fact that (21) is a sub-complex of (24) with the quotient complex

$$
0 \rightarrow \operatorname{Lie}\left(\operatorname{Stab}\left(a_{\infty}\right)\right) \stackrel{0}{\rightarrow} H^{1}\left(T^{2}, i \mathbb{R}\right) \rightarrow 0 .
$$

Therefore, the virtual dimension of $\mathcal{M}_{V} \backslash\left(\bar{\partial}_{\infty}\right)^{-1}\left(U_{\Theta}\right)$ at $x=(A, \psi)$ is

$$
\operatorname{dim}\left(\bar{\partial}_{\infty}\right)^{-1}\left(a_{\infty}\right)+\operatorname{dim} H^{1}\left(T^{2}, i \mathbb{R}\right)-\operatorname{dim} \operatorname{Stab}\left(a_{\infty}\right)=1,
$$

where $\operatorname{dim}\left(\bar{\partial}_{\infty}\right)^{-1}\left(a_{\infty}\right)$ is the virtual dimension of the fiber.

Theorem 3.10. Fix an open set $U$ in $V-\left(T^{2} \times[0, \infty)\right)$. There exists a Baire set $\mathcal{P}_{0}$ of perturbations $\mu$ on $V$ with compact supports in $U$, such that the perturbed SeibergWitten moduli space $\mathcal{M}_{V}^{*} \backslash\left(\bar{\partial}_{\infty}\right)^{-1}\left(U_{\Theta}\right)$ is a smooth, oriented manifold of dimension 1 . Moreover,

$$
\partial_{\infty}: \mathcal{M}_{V}^{*} \backslash\left(\bar{\partial}_{\infty}\right)^{-1}\left(U_{\Theta}\right) \rightarrow \chi\left(T^{2}\right)
$$

is an immersion and transversal to any given immersed curves in $\chi\left(T^{2}\right)$

Proof. The transversality argument is the same as in the closed case, see the proof of Proposition 2.2, namely, we look at the deformation complex (24) for the parametrized moduli space $\mathcal{M}_{V, Z(U, \mathbb{R})}$ to get the transversality for the parametrized 
moduli space $\mathcal{M}_{V, Z(U, i \mathbb{R})}$. We then apply the infinite dimensional version of MorseSmale theory to the projection $\mathcal{M}_{V, Z(U, i \mathbb{R})} \rightarrow Z(U, i \mathbb{R})$, we obtain that, for $\mu$ in a Baire space $\mathcal{P}_{0} \subset Z(U, i \mathbb{R})$, the moduli space $\mathcal{M}_{V, \mu}^{*} \backslash\left(\bar{\partial}_{\infty}\right)^{-1}\left(U_{\Theta}\right)$ is a smooth manifold of dimension given by virtual dimension calculated as above.

We first show that for a generic perturbation $\mu$ (a co-closed imaginary valued 1form with compact support in $U)$, the map $\left(G_{\delta}^{*}, L\right)$ as given by $(23)$ is surjective. At an irreducible monopole $[A, \psi]$ in $\left(\bar{\partial}_{\infty}\right)^{-1}\left(a_{\infty}\right)$ for $a_{\infty} \in \chi\left(T^{2}\right) \backslash U_{\Theta}$, we will show that

$$
L: \quad K e r G_{\delta}^{*} \times Z(U, i \mathbb{R}) \rightarrow \operatorname{Ker} G_{\delta}^{*}
$$

is surjective. Suppose that $\left(\alpha_{1}, \phi_{1}\right)$ is $L_{\delta}^{2}$-orthogonal to the image of the above map, then $(\alpha, \phi)=e_{2 \delta}(t)\left(\alpha_{1}, \phi_{1}\right)$ is $L^{2}$-orthogonal to the image of the above map, hence, $(\alpha, \phi)$ is in $L_{1,-\delta}^{2}$ and satisfies the equations (7) as in the proof of Proposition 2.2. Hence, there is a real valued function $f$ on $V$ (with infinite cylindrical end) such that $\phi=i f \psi, \alpha=-2 i d f$ and

$$
2 d^{*} d f+|\psi|^{2} f=0
$$

$d f \in L_{1,-\delta}^{2}$ implies that on $T^{2} \times[0, \infty), \frac{\partial f}{\partial t}$ is in $L_{-\delta}^{2}$, then by Cauchy-Schwartz inequality

$$
|f(t)-f(0)|^{2} \leq \int_{0}^{t} e^{2 \delta s} d s \int_{0}^{t}\left|e^{-\delta s} \frac{\partial f}{\partial t}(s)\right|^{2} d s
$$

this implies that for $T>>0$,

$$
\int_{\partial V(T)}|f|^{2} \leq C_{0} e^{2 T \delta}\left\|\frac{\partial f}{\partial t}\right\|_{L_{-\delta}^{2}}^{2} \leq C e^{2 T \delta}
$$

for some constants $C_{0}, C .[A, \psi] \in\left(\bar{\partial}_{\infty}\right)^{-1}\left(a_{\infty}\right)$, whose asymptotic behaviour has been studied in the previous subsection, we see that there exist gauge representatives $(A, \psi)$ and $a_{\infty}$ of $[A, \psi]$ and $\left[a_{\infty}\right]$, so that $(A, \psi)$ decays to $\left(a_{\infty}, 0\right)$ exponentially at the rate at least $\frac{\mu_{a_{\infty}}}{2}$, where $\mu_{a_{\infty}}$ is the smallest absolute value of the non-zero eigenvalue of $Q_{a_{\infty}}$ (Cf. (18)). On $T^{2} \times[0, \infty)$, write $\alpha=\pi^{*}\left(a_{\infty}\right)+\alpha^{1}+\alpha^{0} d t$ with $\alpha^{i} \in \Omega^{i}\left(T^{2}, i \mathbb{R}\right)$, using the analysis in Appendix of [25], we get

$$
\left\|\alpha^{0}\right\|_{C^{0}\left(T^{2} \times[T, T+1]\right)} \leq C_{1} e^{-T \frac{\mu_{a_{\infty}}}{2}},
$$

from some constant $C_{1}$. As $-2 i \frac{\partial f}{\partial t}=\alpha^{0}$, we obtain

$$
\left|\frac{\partial f}{\partial t}\right|_{\partial V(T)} \mid \leq C_{2} e^{-T \frac{\mu_{a_{\infty}}}{2}},
$$


for some constant $C_{2}$. From two inequalities (25) and (26), and note that $\delta \leq \mu_{a_{\infty}} / 2$, we have

$$
\begin{aligned}
& \left|\int_{V} d^{*} d\left(f^{2}\right) d v o l\right| \\
\leq & \lim _{T \rightarrow \infty} \int_{\partial V(T)} 2|f| \cdot\left|\frac{\partial f}{\partial t}\right| d v o l \\
\leq & \lim _{T \rightarrow \infty} 2\left(\int_{\partial V(T)}|f|^{2} d v o l\right)^{\frac{1}{2}}\left(\int_{\partial V(T)}\left|\frac{\partial f}{\partial t}\right|^{2} d v o l\right)^{\frac{1}{2}} \\
\leq & \lim _{T \rightarrow \infty} 2 C C_{1} e^{T\left(\delta-\mu_{a_{\infty}} / 2\right)}=0 .
\end{aligned}
$$

Now multiply $2 d^{*} d f+|\psi|^{2} f=0$ by $f$ and integrate it by parts, we get $f=0$, hence $\left(\alpha_{1}, \phi_{1}\right)=0$.

The proof of that $\partial_{\infty}$ is an immersion and transversal to any given immersed curves follows from the Sard-Smale theorem.

An orientation of $\mathcal{M}_{V}$ is obtained from a trivialization of the determinant line bundle of the assembled operator of the deformation complex (24). The trivialization of the determinant line bundle of the complex of (21) is obtained from the orientation of $H_{\delta}^{0}(V) \oplus H_{\delta}^{1}(V)$, the cohomology groups of $\delta$-decaying forms. In fact, we can deform the operator $H_{(A, \psi)}$ with a homotopy $\epsilon \psi, \epsilon \in[0,1]$. The asymptotic operator $Q_{\left[a_{\infty}, 0,0\right]}$ is preserved in the deformation. Thus, if the weight $\delta$ is chosen in such a way that $\delta / 2$ is not in the spectrum of $Q_{\left[a_{\infty}, 0,0\right]}$, then ([17], [23] Lemma 8.3.1) we can ensure that the operator $H_{(A, \epsilon \psi)}$ is Fredholm, for all $\epsilon \in[0,1]$. Since the Dirac operator is complex linear and it preserves the orientation induced by the complex structure on the spinor bundle, a trivialization of the determinant line bundle at $\epsilon=0$ is obtained by the orientation of $H_{\delta}^{0}(V) \oplus H_{\delta}^{1}(V)$. This in turn determines a trivialization of the determinant line for $\epsilon=1$, hence an orientation of $\mathcal{M}_{V}$.

Similar results were obtained by [8] [15].

Now we need to understand the local structure of $\mathcal{M}_{V}^{*}$ around $\left(\bar{\partial}_{\infty}\right)^{-1}(\Theta)$. The center manifold technique developed in [23] is a useful model to study the structure of $\left(\bar{\partial}_{\infty}\right)^{-1}\left(U_{\Theta}\right)$.

We briefly recall a few facts about center manifolds [23]. In general, suppose we are given a system of the form

$$
\dot{x}=Q x+N(x)
$$

with $Q$ a linear operator acting on a Hilbert space $\mathcal{X}$. Assume we also have the decomposition $\mathcal{X}=\mathcal{X}_{h}^{+} \oplus \mathcal{X}_{c} \oplus \mathcal{X}_{h}^{-}$determined by the positive, negative, and zero spectrum of the operator $Q$. Let $\mathcal{X}_{h}=\mathcal{X}_{h}^{+} \oplus \mathcal{X}_{h}^{-}$, and consider the projections $\pi_{c}: \mathcal{X} \rightarrow \mathcal{X}_{c}$ and $\pi_{h}: \mathcal{X} \rightarrow \mathcal{X}_{h}$. We denote by $Q_{h}^{ \pm}$and $Q_{c}$ the induced operators on $\mathcal{X}_{h}^{ \pm}$and $\mathcal{X}_{c}$. By 
construction $Q_{c}$ is trivial. The evolution semigroups $e^{-s Q_{h}^{+}}$and $e^{s Q_{h}^{-}}$, for $s \geq R_{0}>0$, satisfy

$$
\sup _{s \geq R_{0}} \max \left\{e^{\delta s}\left\|e^{-s Q_{h}^{+}}\right\|, e^{-\delta s}\left\|e^{s Q_{h}^{-}}\right\|\right\} \leq C,
$$

for some constant $C>0$. This follows from the bound

$$
\frac{1}{2} \inf \left\{|\lambda| \mid \lambda \in \operatorname{spec}\left(Q_{\Theta}\right), \lambda \neq 0\right\}=\delta>0 .
$$

The center manifold theorem (in [23]) states that there exists a map $\varphi: \mathcal{X}_{c} \rightarrow \mathcal{X}_{h}$ that vanishes to second order at the origin, and such that an element $\tilde{x}(s)$ is a solution of (27) if and only if the projection $\pi_{c} \tilde{x}(s)$ is a solution of the equation

$$
\dot{x_{c}}=\pi_{c} N\left(x_{c}+\varphi\left(x_{c}\right)\right) \text {. }
$$

The center manifold $\mathcal{H}$ is defined as $\mathcal{H}=\left\{x_{c}+\varphi\left(x_{c}\right) \mid x_{c} \in \mathcal{X}_{c}\right\}$.

We now describe explicitly the center manifold and the stable set for the unperturbed equations (16). In this case, we are considering the operator $Q_{\Theta}$, the Hessian of the functional $f$ at the degenerate critical point $\Theta$. The center manifold $\mathcal{H}_{\Theta}$ for the functional $f$ at the degenerate critical point $\Theta$ is a $C^{2}$-manifold which is invariant under the gradient flow of $f$, contains a small neighbourhood $U_{\Theta}$ of $\Theta$, and has tangent space at $\Theta$ given by

$$
\mathcal{H}_{\Theta}^{1}=H^{1}\left(T^{2}, i \mathbb{R}\right) \oplus k e r \bar{\partial}_{\Theta} \oplus k e r \bar{\partial}_{\Theta}^{*} \cong \mathbb{C}^{3}
$$

Lemma 3.11. At every point $x=(a, \alpha, \beta) \in \mathcal{H}_{\Theta}^{1}$, the gradient vector $\nabla f(a, \alpha, \beta)$ is tangent to $\mathcal{H}_{\Theta}^{1}$, hence $\mathcal{H}_{\Theta}^{1}$ is a center manifold of $f$ around $\Theta$.

Proof. Using the natural complex structure on $T^{2}$, we can identify $\mathcal{H}_{\Theta}^{1}$ as the space of constant sections of

$$
\Lambda^{1}\left(T^{2}, i \mathbb{R}\right) \oplus \Lambda^{0,0}\left(T^{2}, \mathbb{C}\right) \oplus \Lambda^{0,1}\left(T^{2}, \mathbb{C}\right)
$$

For $(a, \alpha, \beta) \in \mathcal{H}_{\Theta}^{1}$, we have $\nabla f(a, \alpha, \beta)=\left(-i \bar{\alpha} \beta,-i \bar{\partial}_{a}^{*} \beta, i \bar{\partial}_{a} \alpha\right)$, which is a constant section. Take $\left(z_{1}, z_{2}, z_{3}\right)$ as the coordinates on $\mathcal{H}_{\Theta}^{1} \cong \mathbb{C}^{3}$, we have

$$
\nabla f\left(z_{1}, z_{2}, z_{3}\right)=\left(-\bar{z}_{2} z_{3},-\frac{\bar{z}_{1} z_{3}}{2},-\frac{z_{1} z_{2}}{2}\right)
$$


The downward gradient flow of $f$ on $\mathcal{H}_{\Theta}^{1}$ is given by

$$
\left\{\begin{array}{l}
\frac{\partial z_{1}}{\partial s}=\bar{z}_{2} z_{3}, \\
\frac{\partial z_{2}}{\partial s}=\frac{\bar{z}_{1} z_{3}}{2}, \\
\frac{\partial z_{3}}{\partial s}=\frac{z_{1} z_{2}}{2} .
\end{array}\right.
$$

Note that this gradient flow is invariant under the $U(1)$-action (the constant gauge transformation):

$$
\left(z_{1}, z_{2}, z_{3}\right) \stackrel{e^{i \alpha} \in U(1)}{\longrightarrow}\left(z_{1}, e^{i \alpha} z_{2}, e^{i \alpha} z_{3}\right)
$$

Lemma 3.12. The quantities $\left|z_{2}\right|^{2}-\left|z_{3}\right|^{2},\left|z_{1}\right|^{2}-\left|z_{2}\right|^{2}-\left|z_{3}\right|^{2}$ and $\operatorname{Im}\left(z_{1} z_{2} \bar{z}_{3}\right)$ are preserved under the gradient flow on $\mathcal{H}_{\Theta}$.

Proof. This is a direct calculation using the gradient flow equations (30).

The stable set of $\left(a_{\infty}, 0\right) \in \mathcal{H}_{\Theta}$ is defined to be

$\mathcal{S}_{a_{\infty}}=\left\{x \in \mathcal{H}_{\Theta}\right.$ such that the flowline of (30) starting at $x$ converges to $\left.\left(a_{\infty}, 0\right)\right\}$.

The stable set $\mathcal{S}$ of $f$ in $\mathcal{H}_{\Theta}$ is the union of these $\mathcal{S}_{a_{\infty}}$, for $\left(a_{\infty}, 0\right) \in \mathcal{H}_{\Theta}$.

Lemma 3.13. Let $(a, \phi)=\left(z_{1}, z_{2}, z_{3}\right) \in \mathcal{H}_{\Theta}$. Then $(a, \phi) \in \mathcal{S}$ if and only if we have

$$
\left\{\begin{array}{l}
\left|z_{2}\right|^{2}-\left|z_{3}\right|^{2}=0 \\
\left|z_{1}\right|^{2}-\left|z_{2}\right|^{2}-\left|z_{3}\right|^{2}=\left|a_{\infty}\right|^{2} \geq 0 \\
\operatorname{Im}\left(z_{1} z_{2} \bar{z}_{3}\right)=0
\end{array}\right.
$$

In particular, $(a, \phi)=\left(z_{1}, z_{2}, z_{3}\right) \in \mathcal{S}_{\Theta}$ (the stable set of the point $\Theta$ ) if and only if

$$
\left\{\begin{array}{l}
\left|z_{2}\right|^{2}-\left|z_{3}\right|^{2}=0 \\
\left|z_{1}\right|^{2}-\left|z_{2}\right|^{2}-\left|z_{3}\right|^{2}=0 \\
\operatorname{Im}\left(z_{1} z_{2} \bar{z}_{3}\right)=0
\end{array}\right.
$$

These equations describe a cone over a torus $T^{2}$ with vertex at $\Theta$. Furthermore, $\mathcal{S} \backslash\{(\Theta, 0)\}$ is a 4-dimensional manifold with boundary $\mathcal{S}_{\Theta} \backslash\{(\Theta, 0)\}$.

Proof. It follows from Lemma 3.12 that $(a, \phi) \in \mathcal{S}$ converges to some $\left(a_{\infty}, 0\right)$ as $t \rightarrow \infty$. The equations (32) define a torus over $\Theta$ and, as $\left|a_{\infty}\right|^{2} \rightarrow 0$, points defined by (31) approach points in $\mathcal{S}_{\Theta}$. 
As in [23], the restriction of the gradient flow of $f$ to the center manifold provides a model for the structure of the space of flows with asymptotic values in a small neighbourhood $U_{\Theta}$ of $\Theta$, in the following sense. Given a point $x$ in $\mathcal{H}_{\Theta}$, the stable set $\mathcal{S}_{x}$ at $x$ is defined as

$$
\mathcal{S}_{x}=\left\{y \in \mathcal{H}_{\Theta} \text { such that the flowline starting at } y \text { converges to } x\right\} .
$$

The stable set $\mathcal{S}=\cup_{x \in \mathcal{H}_{\Theta}} \mathcal{S}_{x}$ defines a refinement of the boundary value map, as described in the following commutative diagram:

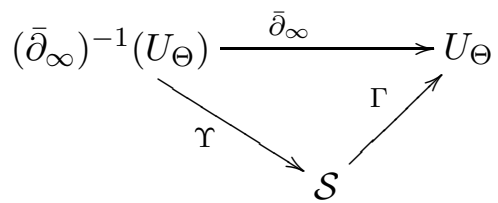

Here $\Upsilon$ is a map defined by taking a flow line on the stable set $\mathcal{S}$ that is exponentially close to a monopole in $\left(\bar{\partial}_{\infty}\right)^{-1}\left(U_{\Theta}\right)$. The map $\Gamma$ is the limit value map under the flow line of $f$ on $\mathcal{S}$.

The results of [23] show that if the projection $\pi_{c} \tilde{x}(s)$ of a flow line $\tilde{x}(s)$ satisfies an estimate

$$
\left\|\partial_{s} \pi_{c} \tilde{x}(s)-\nabla f\left(\pi_{c} \tilde{x}(s)\right)\right\|<C e^{-\delta s},
$$

for all $s \geq R_{0}$, then there exists a unique flow line $x_{c}(s)$ in the center manifold $\mathcal{H}_{\Theta}$ that is exponentially close to $\pi_{c} \tilde{x}(s)$ for large $s \geq R_{0}$, with the same exponent $\delta$ determined by the smallest absolute value of the non-zero eigenvalues of $Q_{\Theta}$. Moreover, for a flow line $\tilde{x}(s)$ satisfying

$$
\left\|\pi_{c} \tilde{x}(s)-a_{\infty}\right\|_{L_{2}^{2}\left(T^{2} \times\{s\}\right)}+\left\|\pi_{h} \tilde{x}(s)\right\|_{L_{2}^{2}\left(T^{2} \times\{s\}\right)} \leq C
$$

for all $s \geq R_{0}$, the projection $\pi_{h} \tilde{x}(s)$ is exponentially small for large $s$, with exponent $\delta$. The condition (34) follows from our explicit construction of the center manifold. This shows that every flow line in $\left(\bar{\partial}_{\infty}\right)^{-1}\left(U_{\Theta}\right)$ is exponentially close to a flow line in the center manifold. Thus, the refinement $\Upsilon$ of the boundary map is well defined and continuous.

The results of the previous discussion and the arguments in [23] (Page 82-100) imply the following structure theorem for our moduli space $\mathcal{M}_{V}$ near $\left(\bar{\partial}_{\infty}\right)^{-1}(\Theta)$.

Theorem 3.14. Fix a metric $g$ and perturbation $P \in \mathcal{P}$ as in Theorem 3.10. Let $\mathcal{K} \subset \mathcal{M}_{V, P}^{*}$ be defined as $\mathcal{K}=\left(\bar{\partial}_{\infty}\right)^{-1}(\Theta)$, and let $\mathcal{K}^{\prime}$ denote the subset $\Upsilon^{-1}(\Theta, 0,0)$, with $\Upsilon$ defined as in (33). Then, generically, the following holds.

(1) $\mathcal{K}^{\prime}$ is empty and $\mathcal{K}$ consists of only finitely many points.

(2) There is a neighbourhood $U_{\Theta}$ of $\Theta$ in $\chi\left(T^{2}\right)$, such that the following holds. The moduli space $\mathcal{M}_{V}^{*} \cap\left(\bar{\partial}_{\infty}\right)^{-1}\left(U_{\Theta}\right)$ is a smooth manifold of dimension 1 , with boundary $\mathcal{K}=\left(\bar{\partial}_{\infty}\right)^{-1}(\Theta)$. 
Proof. By the center manifold theorem in [23], the restriction of any finite energy monopole

$$
[A, \psi] \in \mathcal{M}_{V, \mu}\left(U_{\Theta}\right)=\left(\bar{\partial}_{\infty}\right)^{-1}\left(U_{\Theta}\right)
$$

to the tube $T^{2} \times\left[T_{0}, \infty\right)$ (for a fixed large $T_{0}$ ) is exponentially close to a flow line in the center manifold starting from the point $\Upsilon([A, \psi])$ given by the refinement boundary map (33). The exponential weight is at least a half of the smallest absolute value of the non-zero eigenvalues of $\left(\bar{\partial}_{\Theta}+\bar{\partial}_{\Theta}^{*}\right)$. Theorem 3.10 shows that, for a generic choice of the perturbation, the moduli space $\mathcal{M}_{V}^{*}\left(U_{\Theta}\right)$ is a smooth manifold of dimension 1 , away from $\Upsilon^{-1}\left(\mathcal{S}_{\Theta}\right)$. From the analysis of the center manifold theorem, since $\mathcal{S} \backslash\{(\Theta, 0)\}$ is a 4-manifold with boundary $\mathcal{S}_{\Theta} \backslash\{(\Theta, 0)\}$, we know that generically $\mathcal{K}^{\prime}$, if non-empty, is a smooth manifold of dimension given by the virtual dimension: $\operatorname{dim} \mathcal{M}_{V}^{*}-4=-3$, so $\mathcal{K}^{\prime}$ must be empty and $\mathcal{M}_{V}^{*}\left(U_{\Theta}\right)$ is a smooth oriented 1-dimensional manifold with boundary $\mathcal{K}=\left(\bar{\partial}_{\infty}\right)^{-1}(\Theta)$.

One useful observation that we can derive directly from the analysis of the center manifold is the following estimate of the rate of decay of solutions approaching the singular point $\Theta$.

Remark 3.15. Let $x(s)=(a(s), \alpha(s), \beta(s))$ be an irreducible finite energy solution of the Seiberg-Witten equations on $V$, with asymptotic value $\Theta$, that is, $[x] \in \partial_{\infty}^{-1}(\Theta)$. Then the rate of decay in the $s \rightarrow \infty$ direction is polynomial with

$$
\|(a(s)-\Theta, \alpha(s), \beta(s))\|_{L^{2}\left(T^{2} \times\{s\}\right)} \sim \frac{1}{s} .
$$

\subsection{Proof of Theorem 1.2}

From the discussions in the previous subsection, in order to complete our analysis of the structure of the moduli space $\mathcal{M}_{V}$, we only need to prove the following result.

Lemma 3.16. $\mathcal{M}_{V}^{*}$ is compact except for finitely many open ends limiting to $\chi(V)$, the reducible moduli space of $V$ and, generically, $\bar{\partial}_{\infty}\left(\mathcal{M}_{V}^{*}\right)$ can be made transversal at any interior points to any given finite set of curves in $\chi\left(T^{2}, V\right)$.

Proof. We first analyse the set of reducible solutions of the monopole equations on $V$. The reducible moduli space $\mathcal{M}_{V}^{\text {red }}$ can be identified with the space $\chi(V)$ of deformed flat connections over $V$, modulo gauge transformations, which is diffeomorphic to a circle. The asymptotic value map $\partial_{\infty}$ is simply the restriction map, which is an embedding

$$
\partial_{\infty}: \mathcal{M}_{V}^{r e d}=\chi(V) \hookrightarrow \chi\left(T^{2}, V\right) .
$$


Let $\chi(V) \hookrightarrow \chi\left(T^{2}, V\right)$ be the circle of reducibles on $V$ modulo gauge equivalence, embedded via the restriction map inside the cylinder $\chi\left(T^{2}, V\right)$. Fix a smooth parameterization $a(t)$ of $\chi(V)$, consider the family of Dirac operators $\not_{a(t)}$ on $V$, twisted with the connection $a(t)$. We can perturb $\chi(V)$ such that $\chi(V) \hookrightarrow \chi\left(T^{2}, V\right)$ is away from a small neighbourhood of the singular point $\Theta$, then we know that the Dirac operator $\bar{\partial}_{a(t)}+\bar{\partial}_{a(t)}^{*}$ on $T^{2}$ has trivial kernel. The 3-dimensional Dirac operator $\not_{a(t)}$ $(a(t) \in \chi(V))$ on $V$ may acquire a non-trivial kernel, however, this only happens at finitely many points on $\chi(V)$, for a generic perturbation in $\mathcal{P}_{0}$ (cf. [20] $\S 7$ ). We show that, if these occur, then the irreducible set $\mathcal{M}_{V}^{*}$ has an open end limiting to such points. If the irreducible set $\mathcal{M}_{V}^{*}$ has an open end limiting to the reducible set $\chi(V)$, then the 3-dimensional Dirac operator $\not_{a(t)}$ has a non-trivial kernel: this can be seen by studying the linearization of the spinor part of the Seiberg-Witten equations. On the other hand, suppose that there is a point $a\left(t_{0}\right)$ on $\chi(V)$, where the operator $\not_{a\left(t_{0}\right)}$ acquires a non-trivial kernel. We can proceed as in Section 7.3 of [20] to analyse the local model of the moduli space $\mathcal{M}_{V}=\mathcal{M}_{V}^{*} \cup \chi(V)$ in a neighbourhood of $\left[a\left(t_{0}\right), 0\right]$, which shows that there exists an open end limiting to $a\left(t_{0}\right)$.

Thus, the rest of the proof of compactness for $\mathcal{M}_{V}=\mathcal{M}_{V}^{*} \cup \chi(V)$ is now reduced to the (by now standard) proof of compactness for Seiberg-Witten moduli spaces [13] [16] [22]. Transversality at the interior of the $\bar{\partial}_{\infty}\left(\mathcal{M}_{V}^{*}\right)$ to any given finite set of curves in $\chi\left(T^{2}, V\right)$ can also be achieved by a generic choice of perturbation on $V$.

Thus, we have completed the proof of the structure theorem for $\mathcal{M}_{V}$ (Theorem 1.2).

\section{Gluing of 3-dimensional monopoles}

Now we begin to discuss the gluing theory. Suppose that $V(r)=V-\left(T^{2} \times[r, \infty)\right)$ lies in a closed 3-manifold $Z$ such that $T^{2}$ splits $Z$ into two components (for example, $\left.V(r) \cup_{T^{2}} \nu(K)\right)$. We identify the solutions of the Seiberg-Witten equations on $V$ differing only by those gauge transformations $V$ which can be extended to $Z$, and denote the resulting moduli space by $\mathcal{M}_{V, Z}^{*}$. Then the boundary value map in Theorem 1.2 has a refinement:

$$
\mathcal{M}_{V, Z}^{*} \longrightarrow \chi\left(T^{2}, Z\right)
$$

where the notation $\chi\left(T^{2}, Z\right)$ indicates the moduli space of flat connections on a trivial line bundle over $T^{2}$ modulo the gauge transformations on $T^{2}$ which can be extended to $Z$. This gives a refined boundary value map and the moduli spaces $\mathcal{M}_{V, Z}^{*}$ enjoy all the properties described in Theorem 1.2 for $\mathcal{M}_{V}^{*}$.

Assume that $Z=V(r) \cup_{T^{2}} \nu(K)$ where $\nu(K)$ is a tubular neighbourhood of a knot $K$ in $Z$. We denote by $\chi(\nu(K), Z)$ the moduli space of flat connections on $\nu(K)$ modulo 
the gauge transformations on $\nu(K)$ which can be extended to $Z$. There is a natural map $\chi(\nu(K), Z) \hookrightarrow \chi\left(T^{2}, Z\right)$, which realizes $\chi(\nu(K), Z)$ as a line in the affine space $\chi\left(T^{2}, Z\right)$.

Thus we can define the following fiber product:

$$
\mathcal{M}_{V, Z}^{*} \times_{\chi\left(T^{2}, Z\right)} \chi(\nu(K), Z) .
$$

This is the main object in the gluing Theorem 1.3. We shall present the argument for the case of the homology sphere $Y$. The argument is analogous in the case of $Y_{1}$ and, up to minor modifications that we shall point out, in the case of $Y_{0}$ as well.

Consider a tubular neighborhood $\nu(K) \subset Z$ endowed with a metric with sufficiently large positive curvature inside $\nu(K)$ and flat near the boundary. When stretching the neck in $Z(r)$, using the standard pointwise estimate on the spinor for Seiberg-Witten monopoles we can ensure that, on $\nu(K)$ endowed with an infinite cylindrical end, the only finite energy solutions of the unperturbed Seiberg-Witten equations are reducibles (with vanishing spinor part). Modulo gauge transformations, these correspond to the moduli space of flat connections on $\nu(K)$. In Lemma 4.11, we will show that, if we choose such a metric for $\nu(K) \subset Y$, it is still possible to have a metric with the same properties for $\nu(K) \subset Y_{1}$ and $\nu(K) \subset Y_{0}$.

Recall that we have a splitting of $Y$ along the torus $T^{2}$ as $Y=V \cup_{T^{2}} \nu(K)$, with $\partial V=\partial \nu(K)=T^{2}$. Assume that the metric $g$ on $Y$ is the product metric on a small neighbourhood of $T^{2}$, and can be extended to a metric on $\nu(K)$ with positive scalar curvature. On both $V$ and $\nu(K)$ we consider as underlying Spin structure the one induced from the restriction of the trivial Spin structure on $Y$. This induces a nontrivial Spin structure on $T^{2}$. The corresponding $\operatorname{Spin}^{c}$ structures $\mathfrak{s}^{\prime}, \mathfrak{s}^{\prime \prime}$ on $V$ and $\nu(K)$ have trivial determinant. In gluing the $\operatorname{Spin}^{c}$ structures $\mathfrak{s}^{\prime}$ and $\mathfrak{s}^{\prime \prime}$ on $V$ and $\nu(K)$ we can only obtain the unique trivial $\operatorname{Spin}^{c}$ structure on $Y$ since $Y$ is a homology sphere. The same holds for $Y_{1}$. In the case of $Y_{0}$, the gluing of the trivial structures $\mathfrak{s}^{\prime}$ and $\mathfrak{s}^{\prime \prime}$ on $V$ and $\nu(K)$ by gauge transformations along the common boundary $T^{2}$ provides different $\operatorname{Spin}^{c}$ structures on $Y_{0}$, which are classified by

$$
H^{1}\left(T^{2}, \mathbb{Z}\right) /\left\{\operatorname{Im}\left(H^{1}(V, \mathbb{Z}), H^{1}\left(T^{2}, \mathbb{Z}\right)\right)+\operatorname{Im}\left(H^{1}(\nu(K), \mathbb{Z}), H^{1}\left(T^{2}, \mathbb{Z}\right)\right)\right\} \cong H^{2}\left(Y_{0}, \mathbb{Z}\right) .
$$

Thus, there are a $\mathbb{Z}$-family of $\operatorname{Spin}^{c}$ structures corresponding to $H^{2}\left(Y_{0}, \mathbb{Z}\right) \cong \mathbb{Z}$.

Let $Y(r)=V \cup_{T^{2}}\left([-r, r] \times T^{2}\right) \cup_{T^{2}} \nu(K)$. We can also consider the manifolds $V$ and $\nu(K)$ with infinite cylindrical ends as

$$
V \cup_{T^{2}}\left([0, \infty) \times T^{2}\right), \quad\left((-\infty, 0] \times T^{2}\right) \cup_{T^{2}} \nu(K)
$$

We continue to use the same notation $V$ and $\nu(K)$ for the manifolds with infinite cylindrical ends, as we did in the previous sections. 
The proof of the gluing Theorem 1.3 consists of several steps. First, we show that, upon stretching the neck $[-r, r] \times T^{2}$ to infinity, the Seiberg-Witten monopoles $\left(A_{r}, \psi_{r}\right)$ on $Y(r)$ approach a pair of finite energy solutions $\left(A^{\prime}, \psi^{\prime}\right)$, and $\left(A^{\prime \prime}, 0\right)$ on the two manifolds $V$ and $\nu(K)$ with infinite cylindrical ends. Then we construct a gluing map, under the hypothesis that the gluing takes place away from $\Theta$ in the character variety $\chi\left(T^{2}\right)$. At the end of this section, we justify the assumption that gluing at $\Theta$ can be avoided.

\subsection{Convergence of monopoles on a 3-manifold with a long neck}

We need to introduce some ad hoc assumptions on the class $\mathcal{P}$ of perturbations for the Seiberg-Witten monopoles on $Y(r)$, so that it behaves nicely under the splitting $r \rightarrow \infty$. We consider perturbations of the monopole equations as in (4), induced by the perturbations of the Chern-Simons-Dirac functional. Notice that, if we choose a perturbation with compact support on the manifold $V$ with infinite cylindrical end, this perturbation induces a perturbation on $Y(r)$, for sufficiently large $r>>r_{0}$, which is supported inside the knot complement in $Y(r)$ (which we still denote by $V$ ).

The convergence result we prove in this section depends on a uniform pointwise bound on the solutions $\left(A_{r}, \psi_{r}\right)$ in $\mathcal{M}_{Y(r)}$ which is independent of $r$. The argument for the manifold $Y_{1}(r)$ is the same. The case of the manifold $Y_{0}(r)$ is also analogous, whenever $Y_{0}$ is endowed with a $\operatorname{Spin}^{c}$ structure that restricts to the trivial $\operatorname{Spin}^{c}$ structures on $V$ and $\nu(K)$.

In order to derive the estimates we need, we consider first, for $Y$ a 3-manifold (either without boundary, or with boundary $T^{2}$ ) a functional on the configuration space of $U(1)$-connections and spinors of the form

$$
\mathcal{E}_{Y, \mu}(A, \psi)=\int_{Y}\left(\left|\nabla_{A} \psi\right|^{2}+\frac{\kappa}{4}|\psi|^{2}+\frac{1}{2}\left|F_{A}\right|^{2}+\frac{1}{2}|\sigma(\psi, \psi)+\mu|^{2}\right) d v .
$$

Here we consider compactly supported perturbations $\mu$ of the form described in Section 2 .

Lemma 4.1. If $(A, \psi)$ is a solution of the perturbed $S W$ equations

$$
\left(* F_{A}-\sigma(\psi, \psi)-\mu, \not_{A} \psi\right)=(0,0),
$$

on a compact 3-manifold $Y$ without boundary, then we obtain

$$
\mathcal{E}_{Y, \mu}(A, \psi)=\int_{Y} F_{A} \wedge \mu .
$$

If we consider an open submanifold $Z \subset Y$ with boundary $\partial Z=T^{2}$, such that the perturbation $\mu$ is supported away from $\partial Z$, then for the functional $\mathcal{E}_{Z, \mu}$ we have

$$
\mathcal{E}_{Z, \mathfrak{p}}(A, \psi)=\int_{Z} F_{A} \wedge \mu-\int_{\partial Z}\left\langle\alpha, i \bar{\partial}_{a}^{*} \beta\right\rangle
$$


where we write the connection and spinor as $(a, \alpha, \beta)$ on $T^{2}=\partial Z$. In particular for a cylinder region $Z=T^{2} \times\left[s_{0}, s_{1}\right]$, and perturbation term $\mu$ supported away from $Z$, we have

$$
f\left(a\left(s_{1}\right), \alpha\left(s_{1}\right), \beta\left(s_{1}\right)\right)-f\left(a\left(s_{0}\right), \alpha\left(s_{0}\right), \beta\left(s_{0}\right)\right)=\mathcal{E}_{Z, \mu}(A, \psi),
$$

where we write $(A, \psi)$ in the form $(a(s), \alpha(s), \beta(s))$ on the cylinder $Z$.

Proof. First notice that we have

$$
\int_{Y}\left|\not A_{A} \psi\right|^{2} d v=\int_{Y}\left|\nabla_{A} \psi\right|^{2}+\frac{\kappa}{4}|\psi|^{2}-\frac{1}{2}\left\langle * F_{A} \cdot \psi, \psi\right\rangle .
$$

Here the term $\frac{1}{2}\left\langle * F_{A} \cdot \psi, \psi\right\rangle$ can be written as $-F_{A} \wedge \sigma(\psi, \psi)$. We also have

$$
\begin{gathered}
\int_{Y}\left|* F_{A}-\sigma(\psi, \psi)-\mu\right|^{2} d v= \\
\int_{Y}\left|F_{A}\right|^{2}+|\sigma(\psi, \psi)+\mu|^{2} d v+2 \int_{Y} F_{A} \wedge(\sigma(\psi, \psi)+\mu) .
\end{gathered}
$$

Thus, we can rewrite the functional (36) in the form

$$
\mathcal{E}_{\mu}(A, \psi)=\int_{Y}\left|\not \partial_{A} \psi\right|^{2}+\frac{1}{2}\left|* F_{A}-\sigma(\psi, \psi)-\mu\right|^{2} d v+\int_{Y} F_{A} \wedge \mu .
$$

The identity (37) for a compact manifold then follows. In the case of (38) for $Z$ with $\partial Z=T^{2}$, see the proof of Lemma 3.3 , the boundary term is the difference of

$$
\int_{Z}\left(\left|\partial_{A} \psi\right|^{2}-\left\langle\not_{A}^{*} \not \partial_{A} \psi, \psi\right\rangle\right) d v
$$

and

$$
\int_{Z}\left(\left|\nabla_{A} \psi\right|^{2}-\left\langle\nabla_{A}^{*} \nabla_{A} \psi, \psi\right\rangle\right) d v
$$

The last case (39) for a cylinder follows, since by the assumption on the perturbation term $\int F_{A} \wedge \mu$ is trivial, and the boundary terms give the variation of the functional $f$ along the cylinder.

Notice that the above result allows us to obtain estimates for the $L^{2}$ norms of $\psi$, $\nabla_{A} \psi$, and $F_{A}$.

Lemma 4.2. Suppose we are given solutions $\left(A_{r}, \psi_{r}\right)$ of the perturbed Seiberg-Witten equations (4) on the compact 3-manifold $Y(r)$, with a perturbation $\mu$ supported in the knot complement $V \subset Y(r)$ for all $r \geq r_{0}$. Then we have pointwise bounds

$$
\left|\psi_{r}(y)\right| \leq \kappa(Y), \quad\left|F_{A_{r}}(y)\right| \leq C(\kappa(Y))^{2},
$$

for $y \in Y(r)$, where $C, \kappa(Y)$ are constants independent on $r$. 
Proof. Consider $\kappa(Y(r))=\max _{y \in Y(r)}\{-\kappa(y)+C, 0\}$, where $\kappa(y)$ is the scalar curvature and $C$ is a constant depending only on the perturbation $\mu$. Notice that, by our assumptions on the choice of the perturbation, we can assume that $C$ is independent of $r$. The minimum of the scalar curvature also remains constant upon stretching the cylinder $T^{2} \times[-r, r]$, so that we have $\kappa(Y(r))=\kappa(Y)$ for all $r>0$.

The Weitzenböck formula provides a uniform bound on the spinors in terms of the scalar curvature, namely at a point $y$ where $\left|\psi_{r}(y)\right|$ achieves a maximum we have either $\psi_{r}(y)=0$ or $\left|\psi_{r}(y)\right|^{2} \leq-\kappa(y)+C$. The pointwise bound for the curvature form $F_{A_{r}}$ follows from the bound on $\left|\psi_{r}\right|$ and from the equations.

Using these pointwise estimates and the results of Lemma 4.1, we obtain $L^{2}$ and $L_{1}^{2}$ estimates.

Lemma 4.3. Suppose we are given solutions $\left(A_{r}, \psi_{r}\right)$ of the perturbed Seiberg-Witten equations (4) on the compact 3-manifold $Y(r)$, with a perturbation $\mu$ supported in the knot complement $V \subset Y(r)$, for $r \geq r_{0}$.

(i) Consider an open submanifold $Z \subset Y(r)$, with $\partial Z=T^{2}$ a slice in the product region of $Y(r)$. Then the values $f\left(a_{r}, \alpha_{r}, \beta_{r}\right)$ on $\partial Z$ are uniformly bounded in $r \geq r_{0}$. Here the $\left(a_{r}, \alpha_{r}, \beta_{r}\right)$ are restrictions to $\partial Z$ of the solutions $\left(A_{r}, \psi_{r}\right)$.

(ii) The total variation of the functional $f$ along a cylinder $Z_{r}=T^{2} \times[-r, r] \subset Y(r)$ is uniformly bounded in $r \geq r_{0}$.

Proof. Applying (37) of the previous Lemma together with the assumptions on the perturbation, we obtain

$$
c \leq-\frac{\kappa\left(Y\left(r_{0}\right)\right)}{4}\left\|\psi_{r}\right\|_{L^{2}\left(Y\left(r_{0}\right)\right)}^{2} \leq \mathcal{E}_{Y(r), \mu}\left(A_{r}, \psi_{r}\right)=\int_{Y\left(r_{0}\right)} F_{A_{r}} \wedge \mu \leq C^{\prime},
$$

with $\kappa\left(Y\left(r_{0}\right)\right)=\max (-\kappa(y)+C, 0)$, for $y \in Y\left(r_{0}\right)$. We are using the fact that the scalar curvature satisfies $\kappa \equiv 0$ on the cylinders $T^{2} \times\left[-\left(r-r_{0}\right), r-r_{0}\right]$, and the lower and upper bounds by constants $c, C^{\prime}>0$ independent of $r \geq r_{0}$ follow by the pointwise bound on $\psi_{r}$ and $F_{A_{r}}$. The constant $C^{\prime}$ depends on the perturbation $\mu$.

Now consider the case of a compact set $Z=V \cup_{T^{2}}\left[0, r_{0}\right] \times T^{2}$ in $Y(r)$. Applying (38) of the previous Lemma we estimate

$$
c \leq-\frac{\kappa\left(Y\left(r_{0}\right)\right)}{4}\left\|\psi_{r}\right\|_{L^{2}(Z)}^{2} \leq \mathcal{E}_{Z, \mu}\left(A_{r}, \psi_{r}\right)=\left.f\right|_{\partial Z}+\int_{Z} F_{A_{r}} \wedge \mu
$$

with the boundary term

$$
\left.f\right|_{\partial Z}=f\left(a_{r}, \alpha_{r}, \beta_{r}\right)=-\int_{\partial Z}\left\langle\alpha_{r}, i \bar{\partial}^{*} \beta_{r}\right\rangle d v_{T^{2}} .
$$


By the assumptions on the metric and on the perturbation we know that on $Z^{c}=Y(r) \backslash Z$ we have $\mathcal{E}_{Z^{c}, \mu} \geq 0$, and $\mu \equiv 0$, hence $\mathcal{E}_{Z^{c}, \mu}=\left.f\right|_{\partial Z^{c}}=-\left.f\right|_{\partial Z} \geq 0$. Moreover, for $Z$ of the form as above, we have

$$
-C^{\prime} \leq \int_{Z} F_{A_{r}} \wedge \mu=\int_{Y\left(r_{0}\right)} F_{A_{r}} \wedge \mu \leq C^{\prime}
$$

Thus we have an estimate

$$
c-C^{\prime} \leq f\left(a_{r}, \alpha_{r}, \beta_{r}\right) \leq 0 .
$$

In the case of the cylinder region $Z_{r}$, by considering the two components in the complement $Z_{r}^{c}$ and arguing as above, we obtain uniform bounds on $f\left(a_{r}(r), \alpha_{r}(r), \beta_{r}(r)\right)$ and $f\left(a_{r}(-r), \alpha_{r}(-r), \beta_{r}(-r)\right)$. The variation

$$
f\left(a_{r}(r), \alpha_{r}(r), \beta_{r}(r)\right)-f\left(a_{r}(-r), \alpha_{r}(-r), \beta_{r}(-r)\right)=\mathcal{E}_{Z_{r}, \mathfrak{p}}\left(A_{r}, \psi_{r}\right)
$$

is therefore uniformly bounded in $r \geq r_{0}$.

Lemma 4.4. Suppose we are given solutions $\left(A_{r}, \psi_{r}\right)$ of the perturbed Seiberg-Witten equations (4) on the compact 3-manifold $Y(r)$, with a perturbation $\mu$ supported in the knot complement in $Y(r)$, for $r \geq r_{0}$. Suppose given a compact set $Z$ of the form $V \cup_{T^{2}}\left[0, r_{0}\right] \times T^{2}$ or $\nu(K) \cup_{T^{2}}\left[-r_{0}, 0\right] \times T^{2}$ in $Y(r)$ with $r>r_{0}$. Then we have uniform bounds

$$
\left\|\nabla_{A_{r}} \psi_{r}\right\|_{L^{2}(Z)}^{2} \leq C(\kappa, \mu), \quad\left\|F_{A_{r}}\right\|_{L^{2}(Z)}^{2} \leq C(\kappa, \mu)
$$

where $C(\kappa, \mu)$ is a positive constant, depending on the scalar curvature and on the perturbation, independent of $r \geq r_{0}$.

Proof. In order to derive the estimate for the $L^{2}$-norm of $\nabla_{A} \psi$, we use the result of Lemma 4.3. We have

$$
c \leq\left\|\nabla_{A_{r}} \psi_{r}\right\|_{L^{2}(Z)}^{2}+\int_{Z} \frac{\kappa}{4}\left|\psi_{r}\right|^{2} d v \leq C^{\prime}
$$

and

$$
c \leq \frac{1}{2}\left\|F_{A_{r}}\right\|_{L^{2}(Z)}^{2}+\int_{Z} \frac{\kappa}{4}\left|\psi_{r}\right|^{2} d v \leq C^{\prime} .
$$

Since the second term is uniformly bounded in $r \geq r_{0}$, we obtain the result. 
Notice that the uniform bound on the curvature justifies our choice of the finite energy condition (12) for monopoles on the manifold $V$ with infinite cylindrical end.

Now we can establish the convergence result for the Seiberg-Witten monopoles on $Y(r)$ as $r \rightarrow \infty$.

Proposition 4.5. Assume that the metric on $Y(r)$ and the perturbation are chosen as specified in the beginning of this section. Suppose the moduli spaces $\mathcal{M}_{Y(r)}^{*}\left(\mathfrak{s}_{r}\right)$ are nonempty for $r>>0$, and let $\left(A_{r}, \psi_{r}\right)$ be a solutions representing elements in $\mathcal{M}_{Y(r)}^{*}\left(\mathfrak{s}_{r}\right)$.

(a) For any fixed compact set $Z=V \cup_{T^{2}}\left(T^{2} \times\left(0, r_{0}\right]\right) \subset Y(r)$, there exist gauge transformations $\lambda_{r}$ on $Y(r)$, such that a subsequence of $\lambda_{r}\left(A_{r}, \psi_{r}\right)$ converges smoothly on $Z$ to either a solution $\left(A^{\prime}, \psi^{\prime}\right)$ with $\left[A^{\prime}, \psi^{\prime}\right]$ in $\mathcal{M}_{V}\left(\mathfrak{s}^{\prime}\right)$, or to a solution $\left(a_{\infty}^{\prime \prime}, 0\right)$, with $\left[a_{\infty}^{\prime \prime}, 0\right]$ in $\mathcal{M}_{\nu(K)}^{r e d}\left(\mathfrak{s}^{\prime \prime}\right)=\chi(\nu(K), Y)$.

(b) The solutions $\lambda_{r}\left(A_{r}, \psi_{r}\right)$ restricted to the cylinder $[-r, r] \times T^{2}$ converge smoothly on compact sets to a constant flat connection $a_{\infty}$ on $T^{2}$.

(c) Let $\partial_{\infty}\left[A^{\prime}, \psi^{\prime}\right]=a_{\infty}^{\prime}$ be the asymptotic limit, that is, an element of $\chi\left(T^{2}, V\right)$. Then there exist two gauge transformations $\lambda^{\prime}$ and $\lambda^{\prime \prime}$ on $T^{2}$ that extend to $V$ and $\nu(K)$ respectively, such that we have $\lambda^{\prime \prime} a_{\infty}^{\prime \prime}=\lambda^{\prime} a_{\infty}^{\prime}$ in $\chi\left(T^{2}, Y\right)$.

(d) In the case of $Y_{0}$, we obtain similarly $\lambda^{\prime \prime} a_{\infty}^{\prime \prime}=\lambda^{\prime} a_{\infty}^{\prime}$ in $\chi\left(T^{2}, Y_{0}\right)$. The gauge transformation $\left(\lambda^{\prime}\right)^{-1} \lambda^{\prime \prime}$ over $T^{2}$ determines a cohomology class in $H^{2}\left(Y_{0}, \mathbb{Z}\right)$ which is the element uniquely associated to the $\mathrm{Spin}^{c}$ structure $\mathfrak{s}$ on $Y_{0}$.

\section{Proof.}

(a) Suppose we are given a fixed compact set $Z=V \cup_{T^{2}}\left(T^{2} \times\left(0, r_{0}\right]\right)$ in $Y(r)$. We show that a sequence of elements $\left[A_{r}, \psi_{r}\right]$ of $\mathcal{M}\left(Y(r), \mathfrak{s}_{r}\right)$ has a subsequence that converges smoothly on $Z$ to a solution of the equations. The same result holds for compact sets $Z$ of the form $T^{2} \times\left[-r_{0}, r_{0}\right] \cup_{T^{2}} \nu(K)$. These results were essentially established in [13].

The estimates of Lemma 4.3 and Lemma 4.4 show that there is a uniform bound for the $L^{2}$ norms

$$
\left\|\psi_{r}\right\|_{L^{2}(Z)} \leq C(\kappa, \mu), \quad\left\|F_{A_{r}}\right\|_{L^{2}(Z)} \leq C(\kappa, \mu) .
$$

This implies an $L_{1}^{2}$ bound on the connections

$$
\left\|A_{r}-A_{0}\right\|_{L_{1}^{2}(Z)} \leq \tilde{C} \cdot C(\kappa, \mu)
$$

with the constant $\tilde{C}$ depending on the fixed compact set $Z$, and independent of $r \geq r_{0}$. The bound

$$
\left\|\nabla_{A_{r}} \psi_{r}\right\|_{L^{2}(Z)} \leq C(\kappa, \mu)
$$


of Lemma 4.4, together with the $L^{2}$ bound on the spinors, implies a bound on the $L_{1}^{2}$-norms of the spinors by the elliptic estimate.

Notice that here $Z$ is a compact set of the following form

$$
V \cup_{T^{2}}\left[0, r_{0}\right] \times T^{2} ; \quad \text { or } \quad \nu(K) \cup_{T^{2}}\left[-r_{0}, 0\right] \times T^{2},
$$

thus we have elliptic estimates in the form

$$
\left\|\psi_{r}\right\|_{L_{k}^{2}(Z)} \leq C\left(\left\|\nabla_{A_{r}} \psi_{r}\right\|_{L_{k-1}^{2}\left(Z^{\prime}\right)}+\left\|\psi_{r}\right\|_{L_{k-1}^{2}(Z)}\right)
$$

where $Z^{\prime}$ is a smaller set $Z^{\prime}=V \cup_{T^{2}}\left[0, r_{0}^{\prime}\right] \times T^{2}$, for some $r_{0}^{\prime}<r_{0}$, cf. [20] $\S 4$.1. Since we are only taking estimates on a fixed compact set $Z$ of the form specified above, the constant $C$ in the elliptic estimate depends on $Z$ but does not depend on the parameter $r$ of the underlying manifold $Y(r)$. For the elliptic estimate for the connections, we choose any smooth connection $A_{0}$ on $\operatorname{det}(\mathfrak{s})$ over $Z$ and gauge transformations $\lambda_{r}$ in the identity connected component of the gauge group $\mathcal{G}_{Y(r)}$, such that the forms $\lambda_{r} A_{r}-A_{0}$ are co-closed and annihilate the normal vector at the boundary $T^{2}$. We use an elliptic estimate of the form considered above for the operator $d+d^{*}$. Thus, we can bound the $L_{2}^{2}$-norms of the connections on $Z$, and use a bootstrapping argument to bound the higher Sobolev norms as in [13] [20] [22].

Upon passing to a subsequence, we have obtained elements $\left(A_{r_{i}}, \psi_{r_{i}}\right)$ that converge smoothly on $Z$ to a solution of the equations. This defines a solution $\left(A^{\prime}, \psi^{\prime}\right)$ on $V$ with the cylindrical end $T^{2} \times[0, \infty)$. The case of $\nu(K)$ is analogous. With our choice of metric on $\nu(K)$, a finite energy solution on $\nu(K)$ will necessarily be reducible.

To complete the proof of (a) we need to show that the resulting solution on $V$ with infinite end satisfies the finite energy condition (12). This follows from the curvature estimate in Lemma 4.4

(b) To prove the second claim, consider the elements $x_{r}=\left(A_{r}, \psi_{r}\right)$ restricted to the cylinder $[-r, r] \times T^{2}$. Up to a gauge transformation, they can be written in the form

$$
x_{r}(s)=\left(a_{r}(s), \alpha_{r}(s), \beta_{r}(s)\right) .
$$

The functional $f$ is monotone along the cylinder, with variation

$$
\begin{gathered}
f\left(a_{r}(r), \alpha_{r}(r), \beta_{r}(r)\right)-f\left(a_{r}(-r), \alpha_{r}(-r), \beta_{r}(-r)\right)= \\
\int_{-r}^{r}\left\|\nabla f\left(a_{r}(s), \alpha_{r}(s), \beta_{r}(s)\right)\right\|^{2} d s .
\end{gathered}
$$

By the result of Lemma 4.3, there is a uniform bound, independent of $r$ for the variation of the functional $f$ along the cylinder,

$$
f\left(a_{r}(r), \alpha_{r}(r), \beta_{r}(r)\right)-f\left(a_{r}(-r), \alpha_{r}(-r), \beta_{r}(-r)\right) \leq C .
$$


This uniform bound for

$$
c \leq \mathcal{E}_{Z_{r}, \mu}\left(A_{r}, \psi_{r}\right) \leq C,
$$

with $Z_{r}=T^{2} \times[-r, r]$, gives bounds on compact sets $Z \subset Z_{r}$ for the $L^{2}{ }^{2}$ norms $\left\|\nabla_{A_{r}} \psi_{r}\right\|,\left\|\psi_{r}\right\|,\left\|F_{A_{r}}\right\|$, as well as for the $L^{4}$ norm of the spinor. This is enough to start the bootstrapping argument, with elliptic estimates as before, hence we obtain smooth convergence on compact sets in $Z_{r}$ to a solution of the unperturbed SW equations on $T^{2} \times \mathbb{R}$. Such solution must be a flat connection and the trivial spinor. This implies $\mathcal{E}_{Z_{r}, \mu}\left(A_{r}, \psi_{r}\right) \rightarrow 0$, hence, using again Lemma 4.1 together with the estimate (19), we obtain that the limit is actually a critical point $a_{\infty}$ of $f$.

Thus, up to gauge transformations, the sequence of solutions $\left(A_{r}, \psi_{r}\right)$ has a subsequence $\left(A_{r_{i}}, \psi_{r_{i}}\right)$ that converges smoothly on compact sets to a pair $\left(\left(A^{\prime}, \psi^{\prime}\right),\left(a_{\infty}^{\prime \prime}, 0\right)\right)$. In the asymptotic limit we get

$$
\lim _{s \rightarrow \infty} \lambda^{\prime}\left(A^{\prime}, \psi^{\prime}\right)=\lambda^{\prime} a_{\infty}^{\prime}=\lambda^{\prime \prime} a_{\infty}^{\prime \prime}
$$

In the case of the manifold $Y_{0}$,

$$
\left[\lambda^{\prime} a_{\infty}^{\prime}\right]=\left[\lambda^{\prime \prime} a_{\infty}^{\prime \prime}\right]
$$

in $\chi\left(T^{2}, Y_{0}\right)$ imply that $x_{r}=\left[A_{r}, \psi_{r}\right] \in \mathcal{M}_{Y_{0}(r)}^{*}\left(\mathfrak{s}_{k}\right)$ where $\mathfrak{s}_{k}$ corresponds to the cohomology class

$$
\left[\left(\lambda^{\prime}\right)^{-1} \lambda^{\prime \prime}\right] \in H^{1}\left(T^{2}, \mathbb{Z}\right) / H
$$

for

$$
H=\operatorname{Im}\left(H^{1}(V, \mathbb{Z}), H^{1}\left(T^{2}, \mathbb{Z}\right)\right)+\operatorname{Im}\left(H^{1}(\nu(K), \mathbb{Z}), H^{1}\left(T^{2}, \mathbb{Z}\right)\right) .
$$

This completes the proof of the convergence part of the gluing theorem 1.3 for generators. Namely, we have shown that a gauge class in the moduli space $\mathcal{M}_{Y(r)}(\mathfrak{s})$, for a sufficiently large $r$, and perturbation as prescribed, determines an element in

$$
\mathcal{M}_{V, Y}^{*} \times_{\chi\left(T^{2}, Y\right)} \chi(\nu(K), Y) .
$$

\subsection{Proof of Theorem 1.3}

In this subsection we will construct an approximate monopole on $Y(r)$ from any element in $\mathcal{M}_{V, Y}^{*} \times_{\chi\left(T^{2}, Y\right)} \chi(\nu(K), Y)$, and study the gluing that will produce the corresponding monopole on $Y(r)$ for a sufficiently large $r$.

First, we define a pre-gluing operation, where we splice together solutions in $\mathcal{M}_{V}^{*}$ and $\chi(\nu(K))$ with matching asymptotic values, via a smooth cutoff function. This 
produces an approximate solution $\left(A^{\prime}, \psi^{\prime}\right) \#_{r}\left(a_{\infty}^{\prime \prime}, 0\right)$ of the monopole equations on $Y(r)$ for $\left(\left(A^{\prime}, \psi^{\prime}\right),\left(a_{\infty}^{\prime \prime}, 0\right)\right)$ representing an element in $\mathcal{M}_{V, Y}^{*} \times_{\chi\left(T^{2}, Y\right)} \chi(\nu(K), Y)$.

We can assume that $\left.\left(A^{\prime}, \psi^{\prime}\right)\right|_{T^{2} \times[0, \infty) \subset V}$ is in temporal gauge with asymptotic limit $\left(a_{\infty}, 0\right)$, and there is a gauge transformation $\lambda^{\prime \prime}$ on $\nu(K)$ such that $\lambda^{\prime \prime}\left(a_{\infty}^{\prime \prime}\right)=a_{\infty}$ as a flat connection on $T^{2}$. Let $\left(A^{\prime}, \psi^{\prime}\right)=a_{\infty}+\left(a^{\prime}(s), \psi^{\prime}(s)\right)$ on $T^{2} \times[0, \infty)$. We can choose smooth cutoff functions $\rho_{r}(s)(s \in[-2,2])$ with values in $[0,1]$, satisfying $\rho_{r}(s) \equiv 1$ for $s \in[-2,-1]$ and $\rho_{r}(s) \equiv 0$ for $s \in[1,2]$ with $0 \leq \rho^{\prime}(s) \leq 1$.

Define the pre-gluing map with values in $\mathcal{B}(Y(r))$ by setting

$$
\begin{aligned}
& x_{r}=(A, \psi) \\
= & \left(A^{\prime}, \psi^{\prime}\right) \#_{r}^{0}\left(a_{\infty}^{\prime \prime}, 0\right) \\
= & \left\{\begin{array}{lr}
\left(A^{\prime}, \psi^{\prime}\right) & \text { on } V(r-2) \\
a_{\infty}+\rho_{r}(s) \lambda^{\prime \prime}\left(a^{\prime}(s+r), \psi^{\prime}(s+r)\right) & s \in[-2,2] \\
\lambda^{\prime \prime}\left(a_{\infty}^{\prime \prime}, 0\right) & \text { on } \nu(K)(-r+2)
\end{array}\right.
\end{aligned}
$$

Definition 4.6. An approximate solution is by definition an element in the image of the pre-gluing map (40). We use the notation

$$
\mathcal{M}_{V, Y}^{*}\left(a_{\infty}\right):=\partial_{\infty}^{-1}\left(a_{\infty}\right) \subset \mathcal{M}_{V, Y}^{*}
$$

Then $\mathcal{U}\left(a_{\infty}, r\right)$ is defined to be the image of the pregluing map (40) $\#_{r}^{0}: \mathcal{M}_{V, Y}^{*}\left(a_{\infty}\right) \times\left[a_{\infty}^{\prime \prime}, 0\right] \rightarrow \mathcal{B}(Y(r))$.

In order to show that the approximate solutions in $\mathcal{U}\left(a_{\infty}, r\right)$ can be deformed to actual solutions of the monopole equations on $Y(r)$, we consider the span of eigenvectors corresponding to the small eigenvalues of the linearization operator at the approximate solutions.

Consider the linearization operator of the Seiberg-Witten equations on $Y(r)$ at the approximate solution $\left(A^{\prime}, \psi^{\prime}\right) \#_{r}^{0}\left(a_{\infty}^{\prime \prime}, 0\right)$

$$
H_{\left(A^{\prime}, \psi^{\prime}\right) \#_{r}^{0}\left(a_{\infty}^{\prime \prime}, 0\right)}(f, \alpha, \phi)=\left\{\begin{array}{l}
L_{\left(A^{\prime}, \psi^{\prime}\right) \#_{r}^{0}\left(a_{\infty}^{\prime \prime}, 0\right)}(\alpha, \phi)+G_{\left(A^{\prime}, \psi^{\prime}\right) \#_{r}^{0}\left(a_{\infty}^{\prime \prime}, 0\right)}(f) \\
G_{\left(A^{\prime}, \psi^{\prime}\right) \#_{r}^{0}\left(a_{\infty}^{\prime \prime}, 0\right)}^{*}(\alpha, \phi) .
\end{array}\right.
$$

We also need the linearization operators of the Seiberg-Witten equations on $V$ and $\nu(K)$ with infinite cylindrical ends, as defined in the deformation complex (21), acting on $L^{2}$ forms and spinors:

$$
\begin{aligned}
& H_{\left(A^{\prime}, \psi^{\prime}\right)}(f, \alpha, \phi)=\left\{\begin{array}{l}
L_{\left(A^{\prime}, \psi^{\prime}\right)}(\alpha, \phi)+G_{\left(A^{\prime}, \psi^{\prime}\right)}(f) \\
G_{\left(A^{\prime}, \psi^{\prime}\right)}^{*}(\alpha, \phi)
\end{array}\right. \\
& H_{\left(a_{\infty}^{\prime \prime}, 0\right)}(f, \alpha, \phi)=\left\{\begin{array}{l}
L_{\left(a_{\infty}^{\prime \prime}, 0\right)}(\alpha, \phi)+G_{\left(a_{\infty}^{\prime \prime}, 0\right)}(f), \\
G_{\left(a_{\infty}^{\prime \prime}, 0\right)}^{*}(\alpha, \phi)
\end{array},\right.
\end{aligned}
$$


where the operator $L$ is defined as in $(22)$. We think of $H_{\left(A^{\prime}, \psi^{\prime}\right) \#_{r}^{0}\left(a_{\infty}^{\prime \prime}, 0\right)}$ as acting on the elements $(\alpha, \phi)$ in the $L^{2}$ tangent space of the configuration space over the closed manifold $Y(r)$. We continue to denote by $H_{\left(A^{\prime}, \psi^{\prime}\right)}$ and $H_{\left(a_{\infty}^{\prime \prime}, 0\right)}$ the operators defined in the deformation complex (24) acting on the extended $L^{2}$ spaces of connections and spinors, over $V$ and $\nu(K)$ respectively.

Now we discuss the eigenfunctions corresponding to slowly decaying eigenvalues of these operators. The model for our analysis of the operator $H_{\left(A^{\prime}, \psi^{\prime}\right) \#_{r}^{0}\left(a_{\infty}^{\prime \prime}, 0\right)}$ is based on the work of Capell, Lee, and Miller [3],[4]. With operators differing from a translation invariant operator by exponentially decaying terms, we shall adopt the more general setting as in the work of Nicolaescu, [28].

We use the following result, which is the analogy in our context of Theorem A of [3].

Proposition 4.7. Assume that $a_{\infty}$ is a point in $\chi\left(T^{2}\right)$ away from a small neighbourhood $U_{\Theta}$ of $\Theta$. Let

$$
N(r)=\operatorname{dim} \operatorname{Ker}_{L^{2}}\left(H_{\left(A^{\prime}, \psi^{\prime}\right)}\right)+\operatorname{dim} \operatorname{Ker}_{L^{2}}\left(H_{\left(a_{\infty}^{\prime \prime}, 0\right)}\right)+\operatorname{dim} \operatorname{Ker}\left(Q_{a_{\infty}}\right) .
$$

Then, there exists an $N(r)$-dimensional family of eigenvectors of the operator $H_{\left(A^{\prime}, \psi^{\prime}\right) \#_{r}^{0}\left(A^{\prime \prime}, \psi^{\prime \prime}\right)}$ with eigenvalues satisfying $\lambda(r) \rightarrow 0$ as $r \rightarrow \infty$ at the rate at most $1 / r$. The dimension $N\left(r, r^{-(1+\epsilon)}\right)$ of the span of eigenvectors of the operator $H_{\left(A^{\prime}, \psi^{\prime}\right) \#_{r}^{0}\left(a_{\infty}^{\prime \prime}, 0\right)}$ with eigenvalues $\lambda<r^{-(1+\epsilon)}$ is given by

$$
N\left(r, r^{-(1+\epsilon)}\right)=\operatorname{dim} \operatorname{Ker}_{L^{2}}\left(H_{\left(A^{\prime}, \psi^{\prime}\right)}\right)+\operatorname{dim} \operatorname{Ker}_{L^{2}}\left(H_{\left(a_{\infty}^{\prime \prime}, 0\right)}\right)+\operatorname{dim} \ell_{1} \cap \ell_{2},
$$

where $\ell_{1}$ and $\ell_{2}$ are the two Lagrangian submanifolds in $\operatorname{Ker}\left(Q_{a_{\infty}}\right)=H^{1}\left(T^{2}, \mathbb{R}\right)$, determined by the extended $L^{2}$ solutions of $H_{\left(A^{\prime}, \psi^{\prime}\right)}(\alpha, \phi)=0$ and $H_{\left(a_{\infty}^{\prime \prime}, 0\right)}(\alpha, \phi)=0$.

Proof. In order to prove the first claim it is sufficient to check that elements of $H^{1}\left(T^{2}, \mathbb{R}\right)=\operatorname{Ker}\left(Q_{a_{\infty}}\right)$ give rise to approximate eigenfunctions on $Y(r)$ with slowly decaying eigenvalues, that is, with eigenvalues $\lambda(r)$ satisfying $\lambda(r) \rightarrow 0$ at most like $1 / r$. The first statement is then an analogue, in our case, of Proposition 6.B of [3].

Suppose we are given an element $\xi \in \operatorname{Ker}\left(Q_{a_{\infty}}\right)$. If $\chi(s)$ is a cutoff function supported in $[r / 2-\epsilon, 3 r / 2+\epsilon]$ satisfying $\chi(s) \equiv 1$ on $[r / 2, r]$, we have an estimate

$$
\frac{\left\|\left(\partial_{s}+Q_{a_{\infty}}\right) \chi \xi\right\|_{L^{2}(Y(r))}}{\|\chi \xi\|_{L^{2}(Y(r))}} \leq \frac{C}{r} .
$$

This implies a similar estimate for the operator $H_{\left(A^{\prime}, \psi^{\prime}\right) \#_{r}^{0}\left(a_{\infty}^{\prime \prime}, 0\right)}$ on $Y(r)$, for $r \geq r_{0}$ large enough, since we are assuming that this operator differs from $\partial_{s}+Q_{a_{\infty}}$ by terms that are exponentially small in $r$. This is the setting used in [28]. 
The second part of the statement can be derived from the asymptotically exact sequence

$$
0 \rightarrow \mathcal{K}\left(r^{-(1+\epsilon)}\right) \rightarrow \operatorname{Ker}_{L^{2}}^{e x t}\left(H_{\left(A^{\prime}, \psi^{\prime}\right)}\right) \oplus \operatorname{Ker}_{L^{2}}^{e x t}\left(H_{\left(a_{\infty}^{\prime \prime}, 0\right)}\right) \stackrel{\Delta}{\rightarrow} \ell_{1} \oplus \ell_{2} \rightarrow 0
$$

as in the Main Theorem of [28]. Here $\mathcal{K}\left(r^{-(1+\epsilon)}\right)$ denotes the span of the eigenvectors of $H_{\left(A^{\prime}, \psi^{\prime}\right) \#_{r}^{0}\left(A^{\prime \prime}, \psi^{\prime \prime}\right)}$ with small eigenvalues that decay at a rate of at least $r^{-(1+\epsilon)}$. We use the notation $\operatorname{Ker}_{L^{2}}^{\text {ext }}$ for the extended $L^{2}$-solutions, and $\ell_{i}$ for the asymptotic values of the extended $L^{2}$-solutions.

Proposition 4.7 yields the following.

Corollary 4.8. There are no fast decaying eigenvalues, that is, in our problem $N\left(r, r^{-(1+\epsilon)}\right)=0$. However, there is a non-trivial family of eigenvectors of the linearization $H_{\left(A^{\prime}, \psi^{\prime}\right) \#_{r}^{0}\left(a_{\infty}^{\prime \prime}, 0\right)}$ at the approximate solution $\left(A^{\prime}, \psi^{\prime}\right) \#_{r}^{0}\left(a_{\infty}^{\prime \prime}, 0\right)$, with slowly decaying eigenvalues, satisfying $\lambda(r) \rightarrow 0$ at most like $1 / r$.

Proof. We have

$$
\operatorname{dim} \operatorname{Ker}_{L^{2}}\left(H_{\left(A^{\prime}, \psi^{\prime}\right)}\right)=\operatorname{dim} \operatorname{Ker}_{L^{2}}\left(H_{\left(a_{\infty}^{\prime \prime}, 0\right)}\right)=0
$$

Moreover, for a generic choice of the perturbation of the monopole equations on $V$, the Lagrangian subspaces $\ell_{1}$ and $\ell_{2}$ intersect transversely. Thus, we have $N(r)=\operatorname{dim} \operatorname{Ker}\left(Q_{a_{\infty}}\right)$ and $N\left(r, r^{-(1+\epsilon)}\right)=0$. The previous Proposition shows that the span of eigenvectors with slowly decaying eigenvalues is non-trivial. In fact, it shows the existence of (at least) a two dimensional family parameterized by the elements of $H^{1}\left(T^{2}, \mathbb{R}\right)=\operatorname{Ker}\left(Q_{a_{\infty}}\right)$.

Suppose we are given an element $(a, \phi)$ on $Y(r)$ such that $x_{r}+(a, \phi)$ is a solution of the monopole equations on $Y(r)$. Then $(a, \phi)$ satisfies

$$
H_{x_{r}}(a, \phi)+N_{x_{r}}(a, \phi)+\Sigma\left(x_{r}\right)=0,
$$

where $\Sigma$ is the error term defined by

$$
\Sigma\left(x_{r}\right)=\left(\begin{array}{c}
* F_{A}-\sigma(\psi, \psi)-\mu \\
\not_{A} \psi
\end{array}\right),
$$

as by equation (4), and $N$ is the non-linear term

$$
N_{A, \psi}(a, \phi)=\left(\begin{array}{c}
\sigma(\phi, \phi) \\
a . \phi
\end{array}\right) .
$$


Though we do not treat the more general case in this paper, we mention that one can consider the same argument with an additional perturbation term $P(A, \psi)$. In this case, an additional term $P(A, \psi)$ enters the expression for the error term $\Sigma\left(x_{r}\right)$, and an additional non-linear part $\mathcal{N} P_{A, \psi}$ of the perturbation

$$
\mathcal{N} P_{A, \psi}=P((A, \psi)+(a, \phi))-\mathcal{D} P_{A, \psi}(a, \phi)
$$

is added to the expression of $N_{A, \psi}$. This case will be discussed elsewhere.

Choose $\lambda=\lambda(r)>0$ such that $\lambda(r)$ is not an eigenvalue of $H_{x_{r}}=H_{\left(A^{\prime}, \psi^{\prime}\right) \#_{r}^{0}\left(a_{\infty}^{\prime \prime}, 0\right)}$, for all approximate solutions $x_{r}=\left(A^{\prime}, \psi^{\prime}\right) \#_{r}^{0}\left(a_{\infty}^{\prime \prime}, 0\right)$ in $\mathcal{U}\left(a_{\infty}, r\right)$. Consider the projection maps $\Pi\left(\lambda(r), x_{r}\right)$ onto the span of the eigenvectors of $H_{x_{r}}$ with eigenvalues smaller than $\lambda(r)$.

The condition that, for a given approximate solution $x_{r}$, the element $x_{r}+(a, \phi)$ is an actual solution of monopole equations can be written as a system of two equations:

$$
\begin{gathered}
\Pi\left(\lambda(r), x_{r}\right)\left(N(a, \phi)+\Sigma\left(x_{r}\right)\right)=0 \\
H_{x_{r}}(a, \phi)+\left(1-\Pi\left(\lambda(r), x_{r}\right)\right)\left(N(a, \phi)+\Sigma\left(x_{r}\right)\right)=0 .
\end{gathered}
$$

If the equation (42) admits a unique solution $(a, \phi)$, then the condition that $x_{r}+(\alpha, \phi)$ is a solution of the monopole equations on $Y(r)$ can be rephrased as the condition that (41) is satisfied, with $(a, \phi)$ the unique solution of (42).

The second equation (42) can be written as the fixed point problem

$$
(a, \phi)=-H_{x_{r}}^{-1}\left(1-\Pi\left(\lambda(r), x_{r}\right)\right)\left(N(a, \phi)+\Sigma\left(x_{r}\right)\right) .
$$

The following result proves existence and uniqueness of the solution to (43).

Lemma 4.9. There exists a positive constant $C>0$, such that, if a given approximate solution $x_{r}$ satisfies $\left\|\Sigma\left(x_{r}\right)\right\|_{L^{2}(Y(r))} \leq C \epsilon(r)^{2}$, for some small and positive $\epsilon(r)$ satisfying $\epsilon(r)<\frac{\lambda(r)}{2 C}$, then the map

$$
\mathcal{T}_{r}(a, \phi):=-H_{x_{r}}^{-1}\left(1-\Pi\left(\lambda(r), x_{r}\right)\right)\left(N(a, \phi)+\Sigma\left(x_{r}\right)\right)
$$

maps the ball $B_{\epsilon(r)}=\left\{(a, \phi) \mid\|(a, \phi)\|_{L_{1}^{2}(Y(r))}^{2} \leq \epsilon(r)\right\}$ to itself and is a contraction on $B_{\epsilon(r)}$.

Proof. Let $C>0$ be a constant such that the quadratic term satisfies the estimate

$$
\|N(a, \phi)-N(\tilde{a}, \tilde{\phi})\|_{L^{2}} \leq C\left(\|(a, \phi)\|_{L_{1}^{2}}+\|(\tilde{a}, \tilde{\phi})\|_{L_{1}^{2}}\right)\|(a, \phi)-(\tilde{a}, \tilde{\phi})\|_{L_{1}^{2}},
$$

independent of $r \geq r_{0}$. This follows from the Sobolev multiplication theorem in dimension 3 . 
On the image of $\left(1-\Pi\left(\lambda(r), x_{r}\right)\right)$, the operator $H_{x_{r}}^{-1}$ is bounded with norm bounded by $\lambda(r)^{-1}$. We have an estimate for $(a, \phi) \in B_{\epsilon(r)}$

$$
\begin{aligned}
& \left\|\mathcal{T}_{r}(a, \phi)\right\|_{L_{1}^{2}(Y(r))} \\
\leq & \frac{1}{\lambda(r)}\left\|N(a, \phi)+\Sigma\left(x_{r}\right)\right\|_{L^{2}(Y(r))} \\
\leq & \frac{C \epsilon(r)^{2}}{\lambda(r)}+\frac{\left\|\Sigma\left(x_{r}\right)\right\|_{L^{2}(Y(r))}}{\lambda(r)} \\
\leq & \frac{2 C \epsilon(r)^{2}}{\lambda(r)} \leq \epsilon(r),
\end{aligned}
$$

which implies that $\mathcal{T}_{r}$ maps the ball $B_{\epsilon(r)}$ to itself.

Let $\left(a_{1}, \phi_{1}\right),\left(a_{2}, \phi_{2}\right) \in B_{\epsilon(r)}$, we have

$$
\begin{aligned}
& \left\|\mathcal{T}_{r}\left(a_{1}, \phi_{1}\right)-\mathcal{T}_{r}\left(a_{2}, \phi_{2}\right)\right\|_{L_{1}^{2}(Y(r))} \\
\leq & \frac{1}{\lambda(r)}\left\|N\left(a_{1}, \phi_{1}\right)-N\left(a_{2}, \phi_{2}\right)\right\|_{L^{2}} \\
\leq & \frac{C}{\lambda(r)}\left\|\left(a_{1}, \phi_{1}\right)+\left(a_{2}, \phi_{2}\right)\right\|_{L_{1}^{2}}\left\|\left(a_{1}, \phi_{1}\right)-\left(a_{2}, \phi_{2}\right)\right\|_{L_{1}^{2}} \\
\leq & \frac{2 C \epsilon(r)}{\lambda(r)}\left\|\left(a_{1}, \phi_{1}\right)-\left(a_{2}, \phi_{2}\right)\right\|_{L_{1}^{2}} .
\end{aligned}
$$

Thus, from $\epsilon(r)<\frac{\lambda(r)}{2 C}$ as chosen, we obtain that $\mathcal{T}_{r}$ is a contraction on $B_{\epsilon(r)}$.

Proposition 4.10. For sufficiently large $r \geq r_{0}$, and for all approximate solutions $x_{r}$ in $\mathcal{U}\left(a_{\infty}, r\right)$, there exists a unique solution $(a, \phi)$ of $(42)$, such that equation (41) is trivially satisfied.

Proof. For all approximate solutions $x_{r}$ in $\mathcal{U}\left(a_{\infty}, r\right)$, we have an estimate on the error term

$$
\left\|\Sigma\left(x_{r}\right)\right\|_{L^{2}(Y(r))} \leq C^{\prime} e^{-\delta r}
$$

for $r \geq r_{0}$, which follows from the exponential decay estimate proved in Proposition 3.8. Thus, we can apply Lemma 4.9, with $\lambda(r)=O\left(r^{-(1+\epsilon)}\right)$ and $\epsilon(r)=O\left(e^{-\delta r / 2}\right)$. By Corollary 4.8 we know that, for $\lambda(r)=O\left(r^{-(1+\epsilon)}\right)$, the projection $\Pi\left(\lambda(r), x_{r}\right) \equiv 0$, hence the solution $(a, \phi)$ of $(42)$, provided by Lemma 4.9 also satisfies trivially equation (41).

Thus, the resulting element $x_{r}+(a, \phi)$ is a true monopole solution on $Y(r)$, close to the approximate solution $x_{r}$. This completes the proof of the gluing theorem 1.3. 


\subsection{Metric}

Concerning the metric after surgery, on $\nu(K)$ inside $Y_{1}$ we consider the metric $g_{1}$ as in the following Lemma 4.11, which is due to Liviu Nicolaescu [29].

Let $g=d u^{2}+d v^{2}$ with $\int_{T^{2}} d u \wedge d v=4 \pi^{2}$, where the torus $T^{2}$ is the boundary of the tubular neighbourhood of the knot $\nu(K)$ in $Y$. We introduce a choice of a metric on $\nu(K)$ inside $Y_{1}$, for which we can still derive the result that the moduli space of monopoles on $\nu(K)$ inside $Y_{1}$ consists of the circle of reducibles.

Lemma 4.11. (Nicolaescu) Let $A$ be an element in $S L(2, \mathbb{Z})$. Suppose we are given $\epsilon>0$ sufficiently small. Consider the flat metric on $T^{2}$ given by $g_{0}=A^{*} g$. There exists a constant $c$ and a smooth path $g(s)(s \in \mathbb{R})$ of flat metrics on $T^{2}$ with the following properties:

(i) $g(s) \equiv \frac{1}{\delta^{2}} g_{0}$, for all $s \leq \epsilon$ and $g(s)=g_{1}$ for all $s \geq 1-\epsilon$;

(ii) $g_{1}=g(1)$ is a metric of the form $g_{1}=k_{1} d u^{2}+k_{2} d v^{2}$ with positive constants $k_{i}$;

(iii) The scalar curvature of the metric $\hat{g}:=g(s)+d s^{2}$ on $T^{2} \times \mathbb{R}$ is non-negative;

(iv) The metric $g_{1}$ can be extended to a metric inside the solid torus $\nu(K)$ with positive scalar curvature.

Proof of Nicolaescu's Lemma: Choose a unit vector $\partial_{u}$ with respect to the metric $\frac{1}{c} g_{0}$, and complete it to an oriented orthonormal frame. Let $\left\{\varphi_{1}, \varphi_{2}\right\} \subset \Omega^{1}\left(T^{2}\right)$ be the dual coframe. This is related to $\{d u, d v\}$ by

$$
\varphi_{1}=d u+a_{0} d v \quad \varphi_{2}=k d v
$$

for some positive constant $k>0$.

The path $g(s)$ is defined by requiring that the coframe

$$
\varphi_{1}(s)=d u+a(s) d v \quad \varphi_{2}(s)=k d v
$$

be orthonormal with respect to $g(s)$, where $a(s)$ is a smooth function satisfying $a(s) \equiv 0$ for all $s \geq 1-\epsilon$ and $a(s)=a_{0}$ for all $s \leq \epsilon$. The only conditions that need to be verified are (iii) and (iv).

We have an orthonormal coframe $\left\{\varphi_{0}, \varphi_{1}, \varphi_{2}\right\}$ on $X=T^{2} \times \mathbb{R}$, with respect to the metric $\hat{g}$, with $\varphi_{0}=d s$, and a corresponding orthonormal frame $\left\{e_{0}, e_{1}, e_{2}\right\}$. The Levi-Civita connection is of the form

$$
\Gamma=\left(\begin{array}{ccc}
0 & x & y \\
-x & 0 & z \\
-y & -z & 0
\end{array}\right) \quad x, y, z \in \Omega^{1}(X) .
$$

The Cartan structural equation gives $d \vec{\varphi}=\Gamma \wedge \vec{\varphi}$, with $\vec{\varphi}=\left(\varphi_{0}, \varphi_{1}, \varphi_{2}\right)$. By the expression of $\varphi_{i}$, we have

$$
d \varphi_{0}=d \varphi_{2}=0, \quad d \varphi_{1}=\frac{\dot{a}}{k} \varphi_{0} \wedge \varphi_{2},
$$


hence we obtain

$$
\begin{aligned}
& x \wedge \varphi_{1}+y \wedge \varphi_{2}=0, \\
& \frac{\dot{a}}{k} \varphi_{0} \wedge \varphi_{2}=-x \wedge \varphi_{0}+z \wedge \varphi_{2}, \\
& -y \wedge \varphi_{0}-z \wedge \varphi_{1}=0 .
\end{aligned}
$$

These equations imply

$$
\Gamma=\frac{\dot{a}}{2 k}\left(\begin{array}{ccc}
0 & \varphi_{2} & \varphi_{1} \\
-\varphi_{2} & 0 & \varphi_{0} \\
-\varphi_{1} & -\varphi_{0} & 0
\end{array}\right)
$$

Thus, we can compute the scalar curvature of $\hat{g}=g(s)+d s^{2}$ on $T^{2} \times \mathbb{R}$ which is $3(\dot{a} / k)^{2}$ by direct calculation.

Claim (iv) then follows by noticing that any diagonal metric of the form

$$
g_{1}=k_{1} d u^{2}+k_{2} d v^{2}
$$

realizes the torus $T^{2}$ metrically as the product of two circles of different radii. Each can bound a hemisphere, endowed with a positive scalar curvature metric, thus extending $g_{1}$ to a metric on a solid torus, with positive scalar curvature.

\subsection{Lines in $\chi\left(T^{2}\right)$}

In this subsection we justify why it is sufficient to consider the gluing map in Theorem 1.3 away from the singular point $\Theta \in \chi\left(T^{2}\right)$.

Lemma 4.12. The intersection points $\partial_{\infty} \mathcal{M}_{V}^{*} \cap \chi(\nu(K))$, with $\chi(\nu(K)) \subset \chi\left(T^{2}\right)$ the circle of reducibles for $\nu(K)$ in either $Y, Y_{1}$, or $Y_{0}$, are contained in $\chi\left(T^{2}\right) \backslash U_{\Theta}$, for some neighborhood $U_{\Theta}$ of the singular point $\Theta$. Thus, the gluing of Theorem 1.3 happens away from the reducible point.

Proof. The torus $T^{2}$ inside $Y$ inherits from the trivial Spin structure of $Y$ the nontrivial Spin structure in which both circles (longitude and meridian) bound, that is, the one determined by the element $(1,1)$ in $H^{1}\left(T^{2}, \mathbb{Z}_{2}\right)$. Introduce the coordinates $u$ and $v$ on $H^{1}\left(T^{2}, \mathbb{R}\right)$, defined by the property that, under the projection to $\chi\left(T^{2}\right)$, they satisfy the following condition. For $[A] \in \chi\left(T^{2}\right), v([A])$ is the holonomy around the meridian $m$ and $u([A])$ is the holonomy around the longitude $l$. Under this coordinate system, the singular point $\Theta$ is given by $(1,1)$ in $\chi\left(T^{2}\right)$, and the reducible circle $\chi(V)=\mathcal{M}_{V}^{r e d}$ is given by $\{u=0\}$. Also for the unperturbed Seiberg-Witten equations 
on $\nu(K) \subset Y$, with the metric of non-negative scalar curvature, strictly positive away from the boundary, the reducible circle $\chi(\nu(K), Y)$ is given by

$$
L_{Y}:=\{v=0\} \subset \chi_{0}\left(T^{2}, Y\right) .
$$

Similarly, in the case of $Y_{1}$, choose a metric with a long cylinder $[-r, r] \times T^{2}$, which agrees with the original metric on $Y$ when restricted to the knot complement $V$, and such that the induced metric in the torus neighbourhood $\nu(K)$ is as described in Lemma 4.11 , then the reducible circle $\chi\left(\nu(K), Y_{1}\right)$ is given by

$$
L_{Y_{1}}:=\{v-u=1\} \subset \chi_{0}\left(T^{2}, Y_{1}\right) .
$$

We might have expected that the reducible case would be $L_{Y_{1}}:=\{v-u=0\}$ from the +1 -surgery manifold $Y_{1}$. The shift is due to the fact that +1 -surgery changes the underlying Spin structure $(1,1)$ by tensoring with a flat $\mathbb{Z}_{2}$-bundle of class $(0,1)$ in $H^{1}\left(T^{2}, \mathbb{Z}_{2}\right)$. In the case of $Y_{0}$, the reducible circle $\chi\left(\nu(K), Y_{0}\right)$ for the unperturbed equations is mapped to a circle $\{u=0\} \subset \chi\left(T^{2}\right)$. This is because

$$
\chi\left(T^{2}, \nu(K)\right)=\chi\left(T^{2}, V\right)=\chi\left(T^{2}, Y_{0}\right) \cong \mathbb{R} \times S^{1},
$$

so that $\chi\left(\nu(K), Y_{0}\right)$ consist of a $\mathbb{Z}$-family of circles given by $\{u=2 k, k \in \mathbb{Z}\}$ where $u$ is the coordinate of $\mathbb{R}$ in $\mathbb{R} \times S^{1}$. The gluing map on the fiber product

$$
\mathcal{M}_{V, Y_{0}}^{*} \times_{\chi\left(T^{2}, Y_{0}\right)}\{u=2 k\}
$$

would correspond to the moduli space $\mathcal{M}_{Y_{0}}\left(\mathfrak{s}_{k}\right)$ where $\mathfrak{s}_{k}$ is the Spin ${ }^{c}$ structure with $c_{1}\left(\operatorname{det}_{\mathfrak{s}_{k}}\right) \in H^{2}\left(Y_{0}, \mathbb{Z}\right)$. For the trivial $\operatorname{Spin}^{c}$ structure $\mathfrak{s}_{0}$, there would be a circle of reducible monopoles in $\mathcal{M}_{Y_{0}}\left(\mathfrak{s}_{0}\right)$ resulting from gluing the reducibles $\chi(V, Y) \#\{u=0\}$, we need to introduce a small perturbation inside $\nu(K)$ such that $\chi\left(\nu(K), Y_{0}\right)=\{u=\eta\}$ where $\eta$ is small number in $\mathbb{R}$ for the trivial $\operatorname{Spin}^{c}$ structure $\mathfrak{s}_{0}$.

Clearly, in all the cases, $\chi(\nu(K))$ does not go through the singular points $\left\{\pi^{-1}(\Theta)\right\}$, hence there is no need to consider the gluing map at the singular point $\Theta$. Note that, after perturbing the metric inside a compact set on the manifold $V$ with an infinite cylindrical end, we can make the open ends in $\mathcal{M}_{V}$ not limit to any intersection points of $\chi(V)=\mathcal{M}_{V}^{r e d}=\{u=0\}$ with any circle $\chi(\nu(K))$ for either $Y, Y_{1}$ or $Y_{0}$.

Thus, with our choice of metric as in the previous subsection, and with the choice of perturbation as in Theorem 3.10, we see that the gluing result stated in Theorem 1.3 holds for the manifolds $Y, Y_{1}$, and $Y_{0}$. This completes the proof of Theorem 1.3.

Remark 4.13. Note that gluing the reducible monopoles on $V$ and $\nu(K)$ with matching boundary condition just gives the extension of the flat connections to the whole manifold 
(after a possible gauge transformation). We call this the trivial gluing. The unique reducible point $\theta_{Y}$ in $\mathcal{M}_{Y}$ is obtained by the trivial gluing of the unique intersection point between the lines $L_{Y}=\{v=0\}$ and $\{u=0\}=\pi^{-1}(\chi(V)) \subset \chi\left(T^{2}, Y\right)$. The unique reducible point $\theta_{Y_{1}}$ in $\mathcal{M}_{Y_{1}}$ is obtained by the trivial gluing of the unique intersection point between the lines $L_{Y}=\{v-u=1\}$ and $\{u=0\}=\pi^{-1}(\chi(V)) \subset \chi\left(T^{2}, Y_{0}\right)$.

\section{The geometric triangle and proof of Theorem 1.1}

In the previous section, we showed that the moduli spaces for irreducible monopoles on $Y, Y_{1}$ and $Y_{0}$ are given by the gluing maps on the following fiber products:

$$
\begin{aligned}
& \mathcal{M}_{Y(r)}^{*} \cong \mathcal{M}_{V, Y}^{*} \times_{\chi\left(T^{2}, Y\right)}\{v=0\}, \\
& \mathcal{M}_{Y_{1}(r)}^{*} \cong \mathcal{M}_{V, Y_{1}}^{*} \times_{\chi\left(T^{2}, Y_{1}\right)}\{v-u=1\}, \\
& \mathcal{M}_{Y_{0}(r)}^{*}\left(\mathfrak{s}_{k}\right) \cong \mathcal{M}_{V, Y_{0}}^{*} \times_{\chi\left(T^{2}, Y_{0}\right)}\{u=2 k\}, \quad \text { for } k \neq 0, \\
& \mathcal{M}_{Y_{0}(r)}^{*}\left(\mathfrak{s}_{0}\right) \cong \mathcal{M}_{V, Y_{0}}^{*} \times_{\chi\left(T^{2}, Y_{0}\right)}\{u=\eta\},
\end{aligned}
$$

where $(u, v)$ is the coordinate system on $\chi\left(T^{2}\right)$ and its covering spaces, $\eta>0$ is a small parameter, and $r>>0$ is a sufficiently large number. We can study these moduli spaces on the common character variety $\chi\left(T^{2}, Y_{0}\right)$ which can be identified as a cylinder $\mathbb{R}^{1} \times S^{1}$. Specifically we take it to be the domain (see Figure 1)

$$
\{(u, v) \mid u \in \mathbb{R}, v \in[-1,1]\} /\{(u,-1) \sim(u, 1)\}
$$

in which the lines corresponding to $L_{Y}, L_{Y_{1}}$ and $L_{Y_{0}}\left(\mathfrak{s}_{k}\right)(k \in \mathbb{Z})$ are drawn.

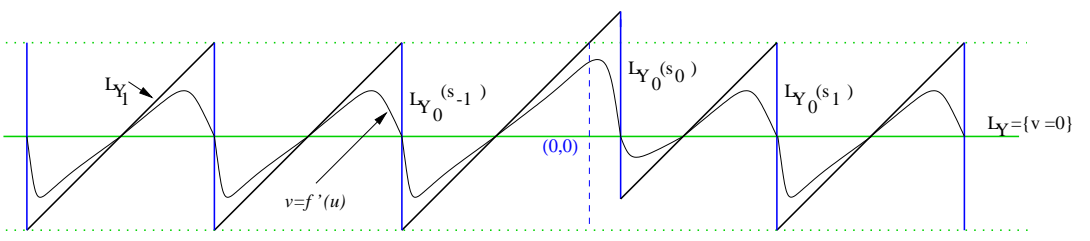

Figure 1: The geometric triangles

In this section, we introduce a suitable perturbation of the curvature equation, supported in the solid torus $D^{2} \times S^{1}$, that simulates the effect of surgery such that the reducible line corresponding to $\nu(K) \subset Y$ is given by the curve $v=f^{\prime}(u)$ as shown in Figure 1.

For a generic perturbation we can assume the curves $\partial_{\infty}\left(M_{V}^{*}\right)$ stay away from the intersection points $\left\{L_{Y} \cap L_{Y_{1}}, L_{Y} \cap L_{Y_{0}}, L_{Y_{1}} \cap L_{Y_{0}}\right\}$, hence $\partial_{\infty}\left(M_{V}^{*}\right)$ is away from small neighbourhood $U$ of those intersection points. Then we can choose a function $f: \mathbb{R} \rightarrow \mathbb{R}$ such that the curve $v=f^{\prime}(u)$ is arbitrarily close to $L_{Y_{1}}$ and $L_{Y_{0}}$ away from the region $U$. This curve is illustrated in Figure 1. The closeness can be measured by a small 
parameter $\epsilon$, such that as $\epsilon \rightarrow 0, v=f^{\prime}(u)$ approaches $L_{Y_{1}}$ and $L_{Y_{0}}$ away from the region $U$. We suppress the dependence of $v=f^{\prime}(u)$ on $\epsilon$.

Fix a $U(1)$-connection $A_{0}$ representing $(0,0)$ on $\chi\left(T^{2}\right)$. For any $U(1)$-connection $A$, define $T_{A}$ to be

$$
T_{z}(A)=-i \int_{\{z\} \times S^{1}}\left(A-A_{0}\right), \quad\left(z \in D^{2}\right) .
$$

Choose a compactly supported 2-form $\mu$ representing the generator of $H_{c p t}^{2}\left(D^{2} \times S^{1}\right)$, such that we have $\int_{D^{2} \times\{p t\}} \mu=1$ for any point on $S^{1}$. Under the isomorphism $H_{c p t}^{2}(\nu(K)) \cong H_{1}(\nu(K))$, given by Poincaré duality, this form corresponds to the generator $[\mu]=P D_{\nu(K)}(l)$. The class of $\mu$ in $H^{2}\left(D^{2} \times S^{1}\right)$ is trivial, and we can write $\mu=d \nu$, where $\nu$ is a 1 -form satisfying $\int_{S^{1} \times\{p t\}} \nu=1$, i.e. $\nu=P D_{T^{2}}(l)$.

Now perturb the Chern-Simons-Dirac functional on $\nu(K) \subset Y(r)$ by adding the term

$$
\int_{D^{2}} f\left(T_{z}(A)\right) \mu
$$

Then the perturbed Seiberg-Witten equations can be written in the following way:

$$
\left\{\begin{array}{l}
F_{A}=* \sigma(\psi, \psi)+f^{\prime}\left(T_{A}\right) \mu \\
\not_{A}(\psi)=0
\end{array} .\right.
$$

Denote the moduli space of (45) (with generic perturbation from $\mathcal{P}_{0}$ ) on $Y(r)$ by $\mathcal{M}_{Y, \mu}$. With respect to the chosen metric on $\nu(K)$, with sufficiently large positive scalar curvature on the support of $\mu$, the only solutions of the perturbed monopole equations on $\nu(K) \subset Y(r)$ are reducibles $(A, 0)$, that satisfy

$$
F_{A}=f^{\prime}\left(T_{A}\right) \mu .
$$

With these preliminary results in place, we can prove the main theorem (Theorem 1.1) of this paper.

Proof of Theorem 1.1. This now follows from the previous discussions and the gluing map (cf Theorem 1.3). From Theorem 1.3 and the surgery perturbation (45) on $\nu(K) \subset Y(r)$, we have

$$
\mathcal{M}_{Y, \mu}^{*} \cong \mathcal{M}_{V, Y}^{*} \times_{\chi\left(T^{2}, Y\right)}\left\{v=f^{\prime}(u)\right\} .
$$

Since we are gluing away from the lattice of $\pi^{-1}(\Theta)$, the limiting points of the open ends of $\mathcal{M}_{V}^{*}$ and the neighbourhood $U$ of the intersections between the character lines, we obtain that solutions of the equations (45) can be identified with

$$
\mathcal{M}_{Y, \mu}^{*} \cong \mathcal{M}_{V}^{*} \times_{\chi\left(T^{2}, Y\right)}\{\text { either } v-u=1 \text {, or } u=2 k, 0 \neq k \in \mathbb{Z} \text { or } u=\eta\},
$$


when the curve $v=f^{\prime}(u)$ is sufficiently close to the line $\{v-u=1\}$ on $\chi\left(T^{2}, Y_{1}\right)$ and the line

$$
\{u=2 k, 0 \neq k \in \mathbb{Z}\}, \quad \text { and }\{u=\eta\}
$$

on $\chi\left(T^{2}, Y_{0}\right)$ (see Figure 1) away from $U$. This shows that

$$
\mathcal{M}_{Y, \mu}^{*} \cong \mathcal{M}_{Y_{1}} \cup \bigcup_{\mathfrak{s}_{k}} \mathcal{M}_{Y_{0}}\left(\mathfrak{s}_{k}\right),
$$

as claimed in Theorem 1.1.

\section{Relative grading}

In this section we show that the grading of the Floer complex $C_{*}(Y, \mu)$, defined with respect to the unique reducible point $\theta_{Y}$, induces compatible gradings on the Floer complexes $C_{*}\left(Y_{1}\right)$ and $C_{*}\left(Y_{0}, \mathfrak{s}_{k}\right)$. The main tools we need in this Section are splitting formulae for the spectral flow, as in [4], [9], [26]. We shall first set up the necessary notation.

On the space $\chi\left(T^{2}, Y_{0}\right)$ whose tangent space at any point is $H^{1}\left(T^{2}, \mathbb{R}\right)$, we introduce the symplectic structure: $(a, b) \mapsto \int_{T^{2}} a \wedge b$, for $a, b \in H^{1}\left(T^{2}, \mathbb{R}\right)$, consider the following Lagrangian submanifolds of $\chi\left(T^{2}, Y_{0}\right)$

$$
\ell_{Y_{1}}=\pi^{*}\left(\partial_{\infty}\left(\mathcal{M}_{\nu(K), Y_{1}}\right)\right)=\left\{(u, v) \in \mathbb{R}^{2} \mid v-u=1\right\}
$$

where

$$
\pi: \chi\left(T^{2}, Y_{0}\right) \rightarrow \chi\left(T^{2}, \nu(K)\right)
$$

is the covering map. We can identify this Lagrangian submanifold with a constant path of Lagrangian subspaces in $H^{1}\left(T^{2}, \mathbb{R}\right)$, given by the tangent spaces along $\ell_{Y_{1}}$, which we denote $\tilde{\ell}_{1}(t)$. Similarly, we can consider the lines

$$
\ell_{Y_{0}}(k)=\{(2 k, v) \mid v \in \mathbb{R}\}
$$

for any fixed $0 \neq k \in \mathbb{Z}$, and

$$
\ell_{Y_{0}}(0)=\{(\eta, v) \mid v \in \mathbb{R}\}
$$

then we have

$$
\cup_{k \in \mathbb{Z}} \ell_{Y_{0}}(k)=\partial_{\infty}\left(\mathcal{M}_{\nu(K), Y_{0}}\right) .
$$

Each Lagrangian submanifold $\ell_{Y_{0}}(k)$ in $\chi\left(T^{2}, Y_{0}\right)$ determines a path $\tilde{\ell}_{Y_{0}}(k)$ of Lagrangian subspaces in the tangent space $H^{1}\left(T^{2}, \mathbb{R}\right)$. 
Moreover, there is a smooth curve

$$
\ell_{\mu}=\pi^{*}\left(\partial_{\infty}\left(\mathcal{M}_{\nu(K), Y_{1}}\right)\right)=\left\{(u, v) \in \mathbb{R}^{2} \mid v=f^{\prime}(u)\right\},
$$

with $\pi: \chi\left(T^{2}, Y_{0}\right) \rightarrow \chi\left(T^{2}, \nu(K)\right)$. We can form smoothly varying Lagrangians of $H^{1}\left(T^{2}, \mathbb{R}\right)$, by taking the tangent space along the curve. We denote the resulting Lagrangians by $\tilde{\ell}_{\mu}$.

Given any choice of two Lagrangians $\tilde{\ell}_{ \pm}$in the tangent space $H^{1}\left(T^{2}, \mathbb{R}\right)$ at the same point on $\chi\left(T^{2}, Y_{0}\right)$ we can define the operators that linearize the monopole equations on the manifolds with boundary $V(r)=V \cup_{T^{2}} T^{2} \times[0,2 r]$ and $\nu(K)(r)=\nu(K) \cup_{T^{2}} T^{2} \times[0,2 r]$. More precisely, for a sufficiently large $r \geq r_{0}$, the gluing theorem gives a splitting $(A, \psi)=\left(A^{\prime}, \psi^{\prime}\right) \#_{r}(a, 0)$, and we can consider the operators (Cf. Section 4.2) on the extended $L_{1}^{2}$ spaces

$$
\begin{aligned}
& H_{\left(A^{\prime}, \psi^{\prime}\right), \tilde{\ell}_{+}}: L_{1}^{2}\left(P_{+} \oplus \tilde{\ell}_{+}\right) \rightarrow L^{2} \\
& H_{\left(A^{\prime}, \psi^{\prime}\right), \tilde{\ell}_{-}}: L_{1}^{2}\left(P_{-} \oplus \tilde{\ell}_{-}\right) \rightarrow L^{2},
\end{aligned}
$$

where $P_{ \pm}$are APS boundary conditions [1] on the extended $L_{1}^{2}$ forms and spinors.

Suppose we are given a path $\tilde{\ell}(\tau)$ of Lagrangians in $H^{1}\left(T^{2}, \mathbb{R}\right)$, which can be written in the form $\tilde{\ell}(\tau)=T_{a(\tau)} \ell$, for some Lagrangian submanifold $\ell$ of $\chi\left(T^{2}, Y_{0}\right)$ with a regular parameterization $a(\tau)$. Assume that, for $0 \leq \tau \leq 1$ the arc $a(\tau)$ of the Lagrangian submanifold $\ell$ avoids the lattice of $\left\{\pi^{-1}(\Theta)\right\}$ and the limiting points of $\partial_{\infty}\left(M_{V}^{*}\right)$ on the circle $\chi(V)$. Moreover, we assume that we have $a$ and $b$ in $\ell \cap \ell_{Y_{1}}$ and that $\ell$ and $\ell_{Y_{1}}$ intersect transversely. Assume the arc of $\ell_{Y_{1}}$ between these endpoints is parameterized over the same interval $0 \leq \tau \leq 1$. Moreover, for small enough $\epsilon$ in the surgery perturbation $\mu$, there are distinct points $a^{\epsilon}$ and $b^{\epsilon}$ in $\ell \cap \ell_{\mu}$. We can assume that, for $\epsilon$ sufficiently small, also $\ell$ and $\ell_{\mu}$ intersect transversely, and there are parameterizations of the arcs of Lagrangians $\ell$ and $\ell_{\mu}$ with endpoints $a^{\epsilon}$ and $b^{\epsilon}$.

We have the following result, which is the key lemma in the comparison of the Maslov indices.

Lemma 6.1. With the hypothesis as above, we have

$$
\operatorname{Maslov}\left(\tilde{\ell}(\tau), \tilde{\ell}_{Y_{1}}\right)=\operatorname{Maslov}\left(\tilde{\ell}(\tau), \tilde{\ell}_{\mu}(\tau)\right),
$$

where the first Maslov index is computed with respect to the parameterizations with endpoints $a$ and $b$, and the second with respect to the parameterizations with endpoints $a^{\epsilon}$ and $b^{\epsilon}$, as specified above.

Proof. By applying the properties of the Maslov index (cf. [5], Section 1), we can see that the claim follows, upon showing that we have

$$
\operatorname{Maslov}\left(\tilde{\ell}_{\mu}(\tau), \tilde{\ell}_{Y_{1}}\right)=0
$$

which is obvious by the choice of $\ell_{\mu}(\tau)$ and $\ell_{Y_{1}}$. 
As a consequence of this result, we obtain the following proposition relating the relative gradings on $\mathcal{M}_{Y, \mu}^{*}$ and $\mathcal{M}_{Y_{1}}^{*}$ respectively. Given a path $\left\{\left(A^{\prime}(\tau), \psi^{\prime}(\tau)\right) \mid \tau \in[0,1]\right\}$, and a corresponding path $\{(a(\tau), 0) \mid \tau \in[0,1]\}$, we can consider the corresponding paths of operators $H_{\left(A^{\prime}(\tau), \psi^{\prime}(\tau)\right)}, H_{(a(\tau), 0)}$, and $H_{\left(A^{\prime}(\tau), \psi^{\prime}(\tau)\right) \#_{r}(a(\tau), 0)}$.

Proposition 6.2. Suppose we are given two irreducible critical points $a=\left[A_{a}, \psi_{a}\right]$ and $b=\left[A_{b}, \psi_{b}\right]$ in $\mathcal{M}_{Y_{1}}^{*}$, and the corresponding elements $a^{\epsilon}=\left[A_{a}^{\epsilon}, \psi_{a}^{\epsilon}\right]$ and $b^{\epsilon}=\left[A_{b}^{\epsilon}, \psi_{b}^{\epsilon}\right]$ in $\mathcal{M}_{Y, \mu}^{*}$. Then we have

$$
\operatorname{deg}_{Y, \mu}\left(a^{\epsilon}\right)-\operatorname{deg}_{Y, \mu}\left(b^{\epsilon}\right)=\operatorname{deg}_{Y_{1}}(a)-\operatorname{deg}_{Y_{1}}(b) .
$$

Proof. Under the pre-gluing map, we can assume that $\left(A_{a}^{\epsilon}, \psi_{a}^{\epsilon}\right)$ and $\left(A_{b}^{\epsilon}, \psi_{b}^{\epsilon}\right)$ are connected by a path $\left(A^{\prime}(\tau), \psi^{\prime}(\tau)\right) \#_{r}(a(\tau), 0)(\tau \in[0,1])$, where we have

$$
\begin{aligned}
& \left(A_{a}^{\epsilon}, \psi_{a}^{\epsilon}\right)=\left(A^{\prime}(0), \psi^{\prime}(0)\right) \#_{r}(a(0), 0), \\
& \left(A_{b}^{\epsilon}, \psi_{b}^{\epsilon}\right)=\left(A^{\prime}(1), \psi^{\prime}(1)\right) \#_{r}(a(1), 0) .
\end{aligned}
$$

Then by definition,

$$
\operatorname{deg}_{Y, \mu}\left(A_{a}^{\epsilon}, \psi_{a}^{\epsilon}\right)-\operatorname{deg}_{Y, \mu}\left(A_{b}^{\epsilon}, \psi_{b}^{\epsilon}\right)=\frac{1}{r^{2}} S F_{Y(r)}\left(H_{\left(A^{\prime}(\tau), \psi^{\prime}(\tau)\right) \#_{r}(a(\tau), 0)}\right),
$$

We can compute this spectral flow with the splitting formula on $Y(r)$ from (Theorem $\mathrm{C}$ of [4]). We obtain

$$
\epsilon S F\left(H_{\left(A^{\prime}(\tau), \psi^{\prime}(\tau)\right), \tilde{\ell}(\tau)}\right)+\operatorname{Maslov}\left(\tilde{\ell}(\tau), \tilde{\ell}_{\mu}\right)+\epsilon S F\left(H_{(a(\tau), 0), \tilde{\ell}_{\mu}}\right) .
$$

With the analogous splitting formula on $Y_{1}(r)$, by applying the Capell-Lee-Miller decomposition of the spectral flow (Theorem $\mathrm{C}$ of [4]), we obtain

$$
\begin{aligned}
& \operatorname{deg}_{Y_{1}}\left(A_{a}, \psi_{a}\right)-\operatorname{deg}_{Y_{1}}\left(A_{b}, \psi_{b}\right) \\
= & \epsilon S F\left(H_{\left(A^{\prime}(\tau), \psi^{\prime}(\tau)\right), \tilde{\ell}(\tau)}\right)+\operatorname{Maslov}\left(\tilde{\ell}(\tau), \tilde{\ell}_{1}\right)+\epsilon S F\left(H_{(a(\tau), 0), \tilde{\ell}_{1}}\right) .
\end{aligned}
$$

In both cases, we can assume that we consider the same boundary value problem (the same choice of Lagrangians) for the operator on the knot complement $V$. We choose $\tilde{\ell}_{\mu}$ or $\tilde{\ell}_{1}$ for the operator on the tubular neighbourhood of the knot $\nu(K)$. The previous Lemma shows that the quantities $\epsilon S F\left(H_{(a(\tau), 0), \tilde{\ell}_{1}}\right)$ and $\epsilon S F\left(H_{(a(\tau), 0), \tilde{\ell}_{\mu}}\right)$ coincide, and that the two Maslov indices are also the same. 
Similarly, we can now compare the relative grading of two solutions in $\mathcal{M}_{Y_{0}}\left(\mathfrak{s}_{k}\right)$ with the relative grading of the corresponding solutions in $\mathcal{M}_{Y, \mu}$.

Again, suppose we are given a path $\tilde{\ell}(\tau)$ of Lagrangians in the tangent space $H^{1}\left(T^{2}, \mathbb{R}\right)$, of the form $\tilde{\ell}(\tau)=T_{a(\tau)} \ell$, for some Lagrangian submanifold $\ell$ of $\chi\left(T^{2}, V\right)$ with a regular parameterization $a(\tau)$. Assume that, for $0 \leq \tau \leq 1$ the arc $a(\tau)$ of the Lagrangian submanifold $\ell$ avoids the lattice of $\left\{\pi^{-1}(\Theta)\right\}$ and the limiting points $\partial_{\infty}\left(\mathcal{M}_{V}^{*}\right)$ on the circle $\chi(V)$. Moreover, we assume that we have $a$ and $b$ in $\ell \cap \ell_{Y_{0}}(k)$ and that $\ell$ and $\ell_{Y_{0}}(k)$ intersect transversely. Assume the arc of $\ell_{Y_{0}}(k)$ between these endpoints is parameterized over the same interval $0 \leq \tau \leq 1$. Moreover, for small enough $\epsilon$ in the surgery perturbation $\mu$, there are points $a^{\epsilon}$ and $b^{\epsilon}$ in $\ell \cap \ell_{\mu}$. We can assume that, for $\epsilon$ sufficiently small, also $\ell$ and $\ell_{\mu}$ intersect transversely, and there are parameterizations of the arcs of Lagrangians $\ell$ and $\ell_{\mu}$ with endpoints $a^{\epsilon}$ and $b^{\epsilon}$.

With these hypothesis we have the following lemma, whose proof is analogous to the proof of Lemma 6.1.

Lemma 6.3. With the hypothesis as above, we have

$$
\operatorname{Maslov}\left(\tilde{\ell}(\tau), \tilde{\ell}_{Y_{0}}(k)\right)=\operatorname{Maslov}\left(\tilde{\ell}(\tau), \tilde{\ell}_{\mu}(\tau)\right),
$$

where the first Maslov index is computed with respect to the parameterizations with endpoints $a_{0}$ and $b_{0}$, and the second with respect to the parameterizations with endpoints $a_{0}^{\epsilon}$ and $b_{0}^{\epsilon}$, as specified above.

We have the following proposition relating the relative gradings on $\mathcal{M}_{Y, \mu}^{*}$ and $\mathcal{M}_{Y_{0}, \mathfrak{s}_{k}}$ $($ for $k \in \mathbb{Z}$ ) respectively.

Proposition 6.4. Suppose we are given $a=\left[A_{a}, \psi_{a}\right]$ and $b=\left[A_{b}, \psi_{b}\right]$ representing two elements in $\mathcal{M}_{Y_{0}, \mathfrak{s}_{k}}$, and let $a^{\epsilon}=\left[A_{a}^{\epsilon}, \psi_{a}^{\epsilon}\right]$ and $b^{\epsilon}=\left[A_{b}^{\epsilon}, \psi_{b}^{\epsilon}\right]$ be the corresponding elements in $\mathcal{M}_{Y, \mu}^{*}$. We have

$$
\operatorname{deg}_{Y_{0}, \mathfrak{s}_{k}}\left(A_{a}, \psi_{a}\right)-\operatorname{deg}_{Y_{0}, \mathfrak{s}_{k}}\left(A_{b}, \psi_{b}\right)=\operatorname{deg}_{Y, \mu}\left(A_{a}^{\epsilon}, \psi_{a}^{\epsilon}\right)-\operatorname{deg}_{Y, \mu}\left(A_{b}^{\epsilon}, \psi_{b}^{\epsilon}\right) \quad \bmod 2 k .
$$

Proof. With the notation as in the Lemma 6.3, we have

$$
\left(A_{a}, \psi_{a}\right)=\left(A^{\prime}(0), \psi^{\prime}(0)\right) \#(a(0), 0)
$$

and

$$
\left(A_{b}, \psi_{b}\right)=\left(A^{\prime}(1), \psi^{\prime}(1)\right) \#(a(1), 0) .
$$


We can calculate the relative grading using the splitting formula on $Y_{0}(r)$

$$
\begin{aligned}
& \operatorname{deg}_{Y_{0}, \mathfrak{s}_{k}}\left(A_{a}, \psi_{a}\right)-\operatorname{deg}_{Y_{0}, \mathfrak{s}_{k}}\left(A_{b}, \psi_{b}\right) \\
= & (\epsilon S F)\left(H_{\left(A^{\prime}(\tau), \psi^{\prime}(\tau)\right), \tilde{\ell}(\tau)}\right)+\operatorname{Maslov}\left(\tilde{\ell}(\tau), \tilde{\ell}_{0}(\tau)\right)+\epsilon S F\left(H_{(a(\tau), 0), \tilde{\ell}_{0}(\tau)}\right)
\end{aligned} .
$$

We can then compare directly these terms with the corresponding terms in the splitting formula for the spectral flow of the operators on $Y(r)$, as in the case of Corollary 6.2. The result of Lemma 6.3 guarantees that we obtain the same result.

Notice that the results of Lemma 6.3 and Corollary 6.4 imply that the grading

$\operatorname{deg}_{Y, \mu}$ defines a choice of an integer lift of the $\mathbb{Z}_{2 k}$-valued relative grading of $C_{*}\left(Y_{0}, \mathfrak{s}_{k}\right)$ given by

$$
\operatorname{deg}_{Y_{0}, \mathfrak{s}_{k}}\left(A_{a}, \psi_{a}\right)-\operatorname{deg}_{Y_{0}, \mathfrak{s}_{k}}\left(A_{b}, \psi_{b}\right)=\operatorname{deg}_{Y, \mu}\left(A_{a}, \psi_{a}\right)-\operatorname{deg}_{Y, \mu}\left(A_{b}, \psi_{b}\right)
$$

under the identification $\mathcal{M}_{Y_{0}, \mathfrak{s}_{k}} \hookrightarrow \mathcal{M}_{Y, \mu}$. We will discuss the properties of the integer lift $C_{(*)}\left(Y_{0}, \mathfrak{s}_{k}\right)$ of $C_{*}\left(Y_{0}, \mathfrak{s}_{k}\right)$ elsewhere.

\section{References}

[1] M.F. Atiyah, V.K. Patodi, I.M. Singer, Spectral asymmetry and Riemannian geometry, I,II,III; Math. Proc. Cambridge Phil. Soc. 77 (1975) 43-69; 78 (1975) 405-432; 79 (1976) 71-99.

[2] P.J. Braam, S.K. Donaldson, Floer's work on instanton homology, knots and surgery, Floer Memorial Volume, Progress in Mathematics, Vol. 133; Birkhäuser 1995.

[3] S.E. Cappell, R. Lee, E.Y. Miller, Self-adjoint elliptic operators and manifold decompositions. I: Low eigenmodes and stretching, Commun. Pure Appl. Math. 49, No.8, 825-866 (1996).

[4] S.E. Cappell, R. Lee, E.Y. Miller, Self-adjoint elliptic operators and manifold decompositions. II: Spectral flow and Maslov index, Commun. Pure Appl. Math. 49, No.9, 869-909 (1996).

[5] S.E. Cappell, R. Lee, E.Y. Miller, On the Maslov index, Comm. Pure Appl. Math., Vol. XLVII (1994) 121-186.

[6] A.L. Carey, J. McCarthy, B.L. Wang, R.B. Zhang, Seiberg-Witten Monopoles in Three Dimensions, Lett. in Math. Phys., Vol. 39, 213-228, 1997;

[7] A.L. Carey, B.L. Wang, Seiberg-Witten-Floer homology and gluing formulae, to appear.

[8] W. Chen, Dehn surgery formulae for Seiberg-Witten invariants of homology 3spheres, preprint, dg-ga/9703009. 
[9] M. Daniel, P.Kirk, with an appendix by K.P. Wojciechowski, A general splitting formula for the spectral flow, Michigan Math. J. 46 (1999), no. 3, 589-617.

[10] D. Freed, K. Uhlenbeck, Instantons and 4-manifolds, MSRI Lecture notes, SpringerVerlag, 1984.

[11] K.A. Froyshov, The Seiberg-Witten equations and four-manifolds with boundary, Math. Res. Lett. 3 (1996), N.3, 373-390;

[12] P.B. Kronheimer, Embedded surfaces and gauge theory in three and four dimensions, Surveys in differential geometry, Vol. III (1996), 243-298, Int. Press, 1998.

[13] P.B. Kronheimer, T.S.Mrowka, The genus of embedded surfaces in the projective plane, Math. Res. Lett. 1 (1994), 797-808.

[14] P.B. Kronheimer, T.S.Mrowka, Monopoles and contact structures. Inventiones Mathematicae, 130 (1997), no. 2, 209-255.

[15] Y. Lim, Seiberg-Witten moduli spaces for 3-manifolds with cylindrical-end $T^{2} \times \mathbb{R}^{+}$. Comm. in Comtemp. Math., Vol. 2, No. 4 (2000) 461-509.

[16] Y. Lim, Seiberg-Witten invariants for 3 -manifolds in the case $b_{1}=0$ or 1 . Pacific J. Math. 195 (2000), no. 1, 179-204.

[17] R.B. Lockhard, R.C. Mc Owen Elliptic operators on non-compact manifolds, Ann. Sci. Norm. Sup. Pisa, IV-12 (1985), 409-446.

[18] M. Marcolli, Seiberg-Witten-Floer Homology and Heegaard Splittings Intern. Jour. of Maths., Vol 7, No. 5 (1996) 671-696.

[19] M. Marcolli, Seiberg-Witten gauge theory, Texts and Readings in Mathematics, Vol. 17, Hindustan Book Agency, New Delhi, 1999.

[20] M. Marcolli, B.L. Wang Equivariant Seiberg-Witten-Floer homology, Commun. Anal. Geom. Vol.9 N.3 (2001) 451-639.

[21] G. Meng, C. H. Taubes $\underline{S W}=$ Milnor Torsion, Math. Res. Lett. 3 (1996), no. 5, 661-674;

[22] J.W. Morgan, The Seiberg-Witten equations and applications to the topology of smooth four-manifolds, Princeton 1996.

[23] J.W. Morgan, T.S. Mrowka and D. Ruberman, The $L^{2}$-moduli space and a vanishing theorem for Donaldson polynomial Invariants, Monographs in Geometry and Topology, Vol 2, 1994.

[24] J.W. Morgan, Z. Szabo and C.H. Taubes, A product formula for the Seiberg-Witten invariants and the generalized Thom Conjecture, J. Differential Geom. 44 (1996), no. 4, 706-788.

[25] T.S. Mrowka, A Mayer-Vietoris principle for Yang-Mills moduli spaces, Ph.D. thesis, (Berkeley), 1988. 
[26] L. Nicolaescu, The Maslov index, the spectral flow, and decompositions of manifolds, Duke Math. J. 80 (1995) no. 2, 485-533.

[27] L.I. Nicolaescu, Lattice points, Dedekind-Rademacher sums and a conjecture of Kronheimer and Mrowka, preprint, math.DG/9801030.

[28] L. Nicolaescu, On the Capell-Lee-Miller gluing theorem, preprint, math.DG/9803154.

[29] L. Nicolaescu, private communication.

[30] L. Simon, Asymptotics for a class of non-linear evolution equations with applications to geometric problems, Annals of Math. (2) 118, (1983) N.3, 525-571.

[31] C.H. Taubes, The stable topology of self-dual moduli spaces, J.Diff.Geom. 29 (1989) 163-230.

[32] C.H. Taubes, The Seiberg-Witten invariants and 4-manifolds with essential tori, Geom. Topol. 5 (2001), 441-519.

[33] C.H. Taubes, private communication.

[34] C.H. Taubes, Gauge theory on asymptotically periodic 4-manifolds, J.Diff.Geom. 25 (1986) 363-430.

[35] R.G. Wang, On Seiberg-Witten Floer invariants and the Generalized Thom Problem, preprint.

\section{A.L. Carey,}

School of Mathematical Sciences, Australian National University, Canberra ACT, Australia acarey@wintermute.anu.edu.au

\section{Marcolli,}

Max-Planck-Institut für Mathematik, D-53111 Bonn, Germany

marcolli@mpim-bonn.mpg.de

\section{B.L. Wang,}

Department of Pure Mathematics, University of Adelaide, Adelaide SA 5005

bwang@maths.adelaide.edu.au 